\title{
LATERAL-TORSIONAL VIBRATION OF A SIDE-LOADED ROTOR WITH ASYMMETRIC SHAFT STIFFNESS
}

\author{
A Thesis \\ presented to \\ the Faculty of California Polytechnic State University, \\ San Luis Obispo
}

In Partial Fulfillment

of the Requirements for the Degree

Master of Science in Mechanical Engineering

by

Clinton Judd

May 14, 2010 
(C) 2010

Clinton Judd

\section{ALL RIGHTS RESERVED}




\section{Committee Membership}

TITLE: $\quad$ LATERAL-TORSIONAL VIBRATION OF A SIDE-LOADED

ROTOR WITH ASYMMETRIC SHAFT STIFFNESS

AUTHOR: $\quad$ Clinton Judd

DATE SUBMITTED:

May 14, 2010

COMMITTEE CHAIR:

Xi Wu, Assistant Professor Mechanical Engineering

COMMITTEE MEMBER: Jim Meagher, Professor Mechanical Engineering

COMMITTEE MEMBER: Tom Mase, Professor Mechanical Engineering 


\section{Abstract \\ LATERAL-TORSIONAL VIBRATION OF A SIDE-LOADED ROTOR WITH ASYMMETRIC SHAFT STIFFNESS \\ By Clinton Judd}

Using energy equations a four degrees of freedom analytical model is developed for a two-disk rotor with shaft stiffness asymmetry. A radial constant force is applied to the outboard disk to emphasize the effects of gravity or aerodynamic side loading. Special emphasis is placed on characterizing the lateral and torsional vibration trends associated with shaft asymmetry which may be used to identify failing shafts in operational rotor systems. Simulation reveals distinct patterns in lateral and torsional response, with strong dependencies on the magnitude of the side load, magnitude of the asymmetry and proximity of the lateral and torsional natural frequencies. Notable interaction is also observed between the lateral and torsional response. Lateral response peaks are found to correlate to torsional response peaks under some conditions. An experiment is performed to compare the response of a real system with the simulated model. 


\section{Acknowledgements}

The author wishes to thank the faculty and staff of the Mechanical Engineering Department for their strong support and encouragement throughout his undergraduate and graduate education. He is especially grateful for the moral support provided during his diagnosis and successive treatment for Hodgkin's Lymphoma during his last quarter at Cal Poly. 


\section{Table of Contents}

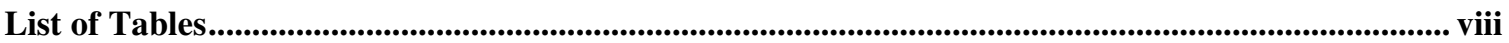

List of Figures ........................................................................................................................................................... ix

Chapter I Introduction .................................................................................................................................... I

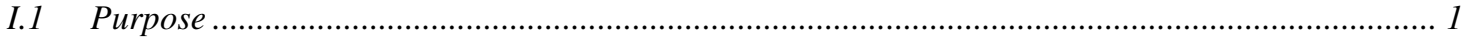

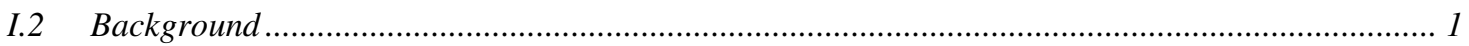

Chapter II Rotor Model ............................................................................................................................ 3

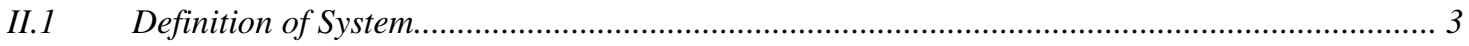

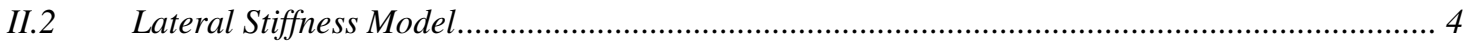

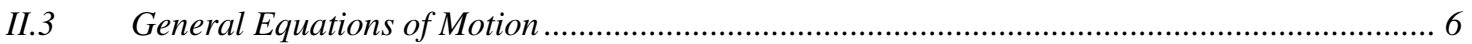

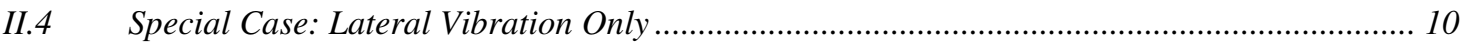

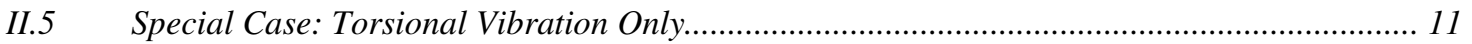

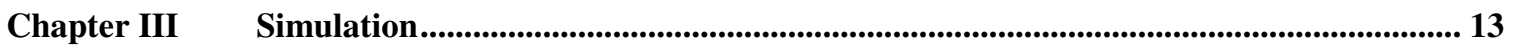

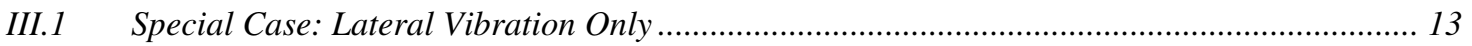

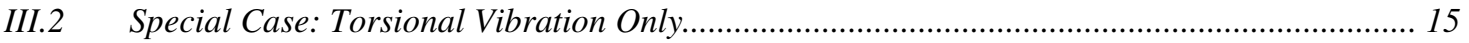

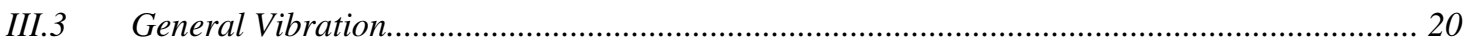

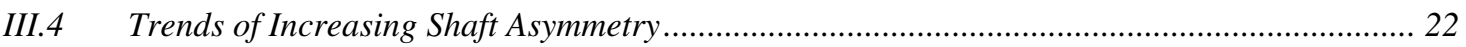

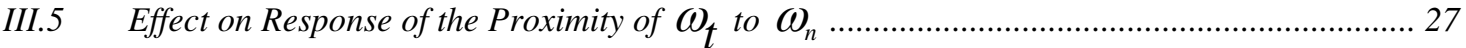

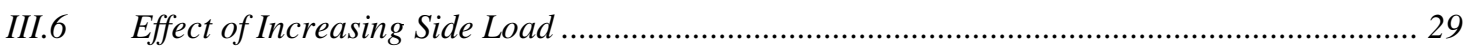

Chapter IV Experiment ............................................................................................................................... 31

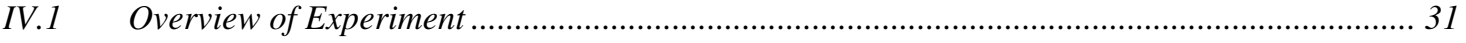

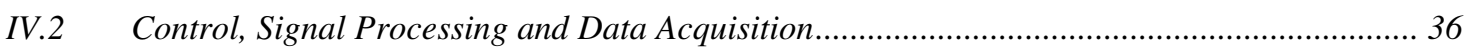

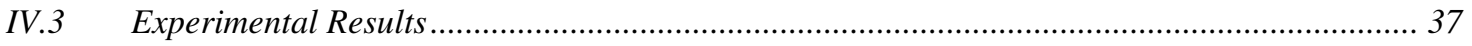

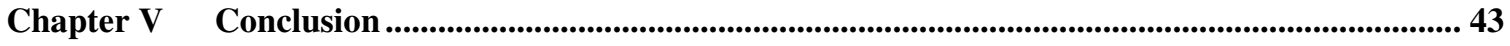

Bibliography.................................................................................................................................................................. 46

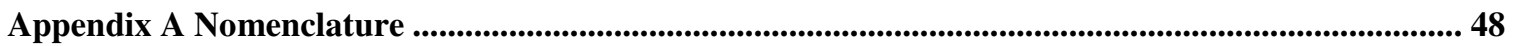

Appendix B Detailed Calculations ................................................................................................................. 49 


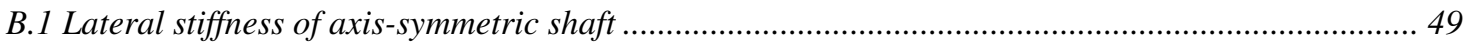

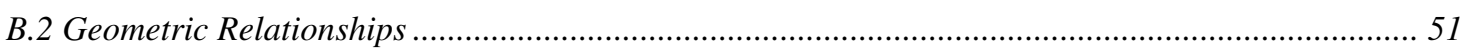

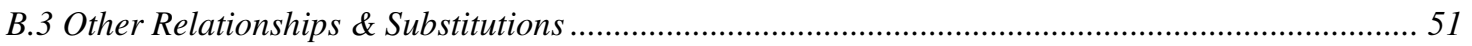

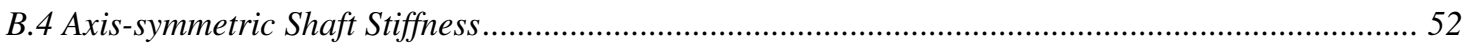

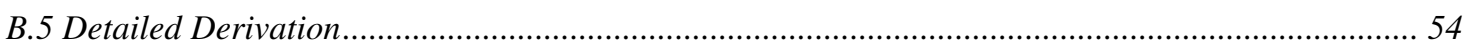

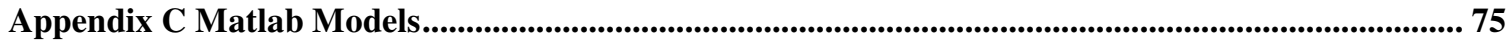

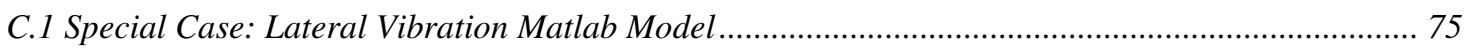

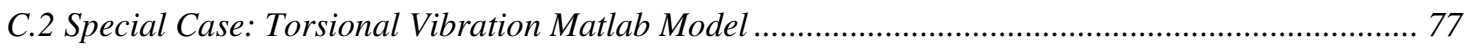

C.3 Special Case: Torsional Vibration Matlab Model For Parametric Study Of $\frac{P_{Y}}{k} / \varepsilon$....................... 79

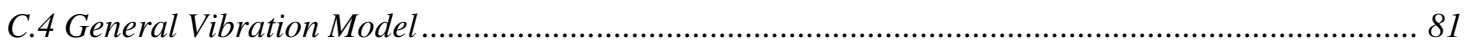

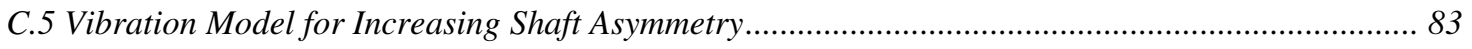

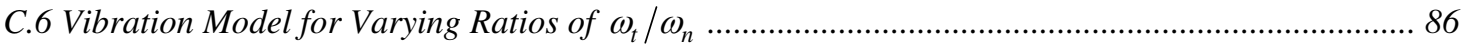

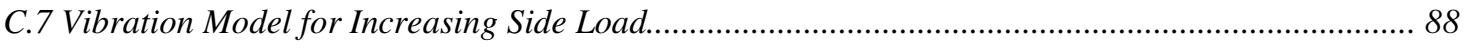

Appendix D Experiment Trial Notes ............................................................................................ 91

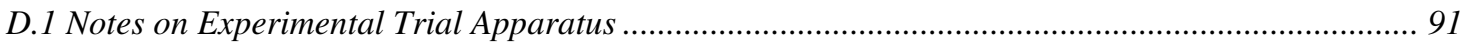




\section{List of Tables}

Table 1: Parameters used for lateral vibration model ...................................................................... 14

Table 2: Parameters used for torsional vibration model ................................................................ 16

Table 3: Parameters used for torsional vibration model for gravitational loading................................ 19

Table 4: Parameters used for general vibration model …......................................................... 21

Table 5: Parameters used in vibration model of rotor with increasing shaft asymmetry ......................... 22 


\section{List of Figures}

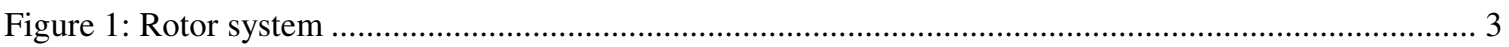

Figure 2: Rotor system model (damping not shown) …….................................................................. 4

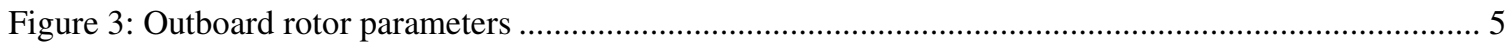

Figure 4: Lateral vibration amplitudes predicted by model..................................................................... 14

Figure 5: Orbit response at half the lateral natural frequency $\left(\Omega=\frac{1}{2} \omega_{n}=1750 \mathrm{rpm}\right)$................................ 15

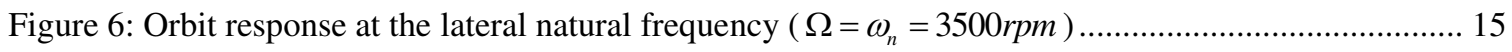

Figure 7: Torsional vibration amplitudes predicted by model.............................................................. 16

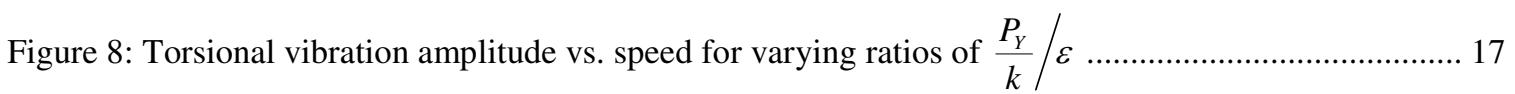

Figure 9: Torsional response at half the torsional natural frequency $\left(\Omega=\frac{1}{2} \omega_{t}=1750 \mathrm{rpm}\right) \ldots \ldots \ldots \ldots \ldots \ldots \ldots \ldots . . . . . . . . .18$

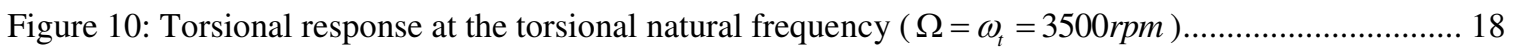

Figure 11: Torsional vibration amplitude vs. speed for gravitationally loaded rotor ................................. 19

Figure 12: Response amplitudes predicted by general vibration model ................................................ 21

Figure 13: Vertical vibration amplitudes for rotor with increasing shaft asymmetry .................................... 23

Figure 14: Torsional vibration amplitudes for rotor with increasing shaft asymmetry ............................... 23

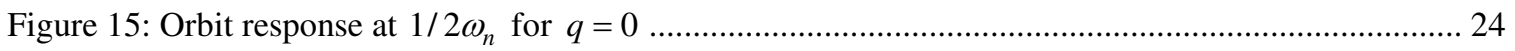

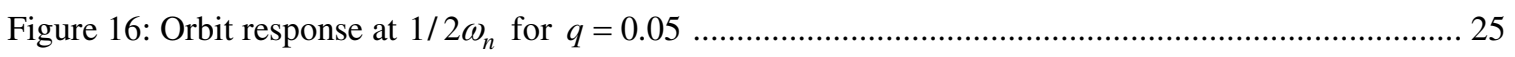

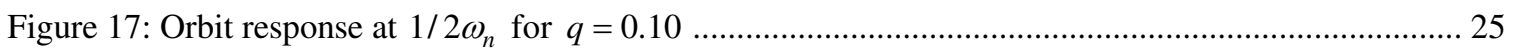

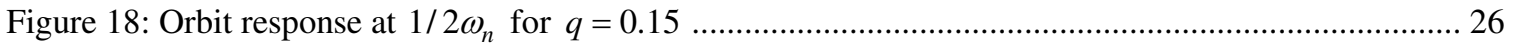

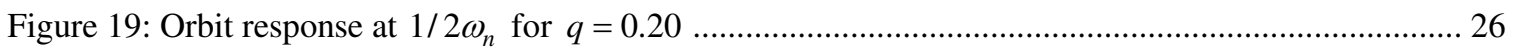

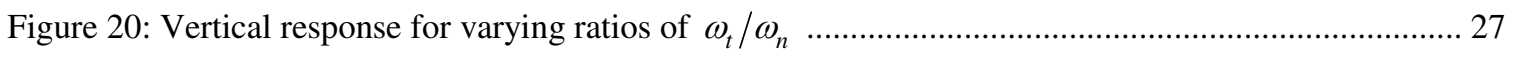

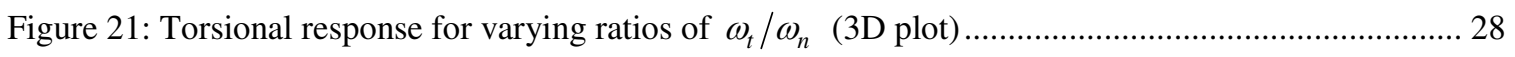

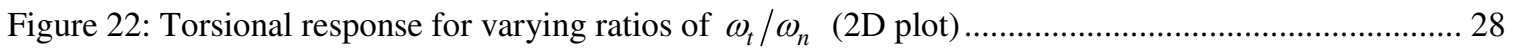

Figure 23: Lateral response for increasing side load (3D plot)............................................................. 29 
Figure 24: Torsional response for increasing side load (3D plot) .............................................................. 30

Figure 25: Bently Nevada RK-4 Rotor Kit configured for a side loaded rotor............................................. 31

Figure 26: Rotor kit configured to minimize torsional natural frequency ............................................... 32

Figure 27: Side load provided by gravity through a pulley system ......................................................... 32

Figure 28: Precision ground flat in shaft to produce asymmetric stiffness.................................................. 33

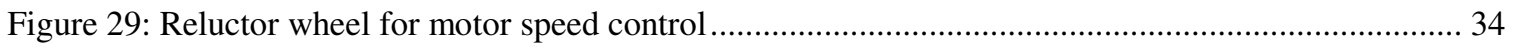

Figure 30: Proximity probes for lateral vibration measurement ............................................................... 34

Figure 31: Torsional transducer consisting of a gear wheel and two proximity probes .............................. 35

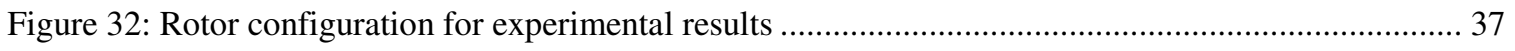

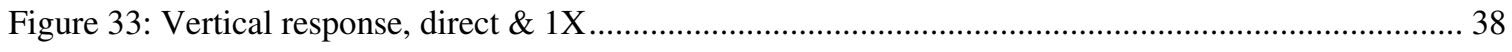

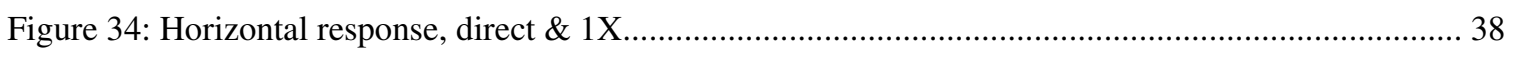

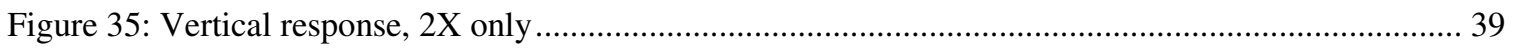

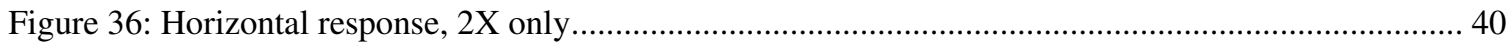

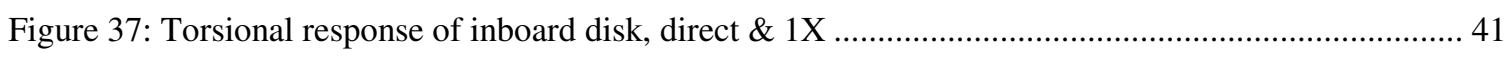

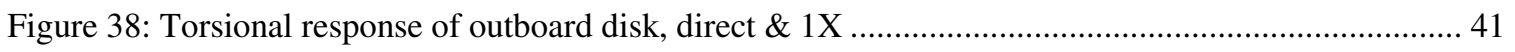

Figure 39: Overall rotor torsional response (difference between inboard \& outboard), $1 \mathrm{X} \& 2 \mathrm{X} \ldots \ldots \ldots \ldots . . . . .42$ 


\section{Chapter I Introduction}

\section{I.1 Purpose}

Modern high speed rotating machinery is constantly driven to the limits of design, materials, and service life. Equipment failure can be costly and hazardous. Diagnostic tools are increasingly important for early detection of component failure. One area of potential failure is in the shaft of a rotating machine which may crack as a result of fatigue. This study aims to model and study the effects of a compromised shaft on rotordynamic behavior in an effort to identify warning signs that could be used to identify a developing failure.

\section{I.2 Background}

A comprehensive literature survey of various crack modeling techniques and system behavior of cracked rotor was given by Wauer (1).This paper contains the modeling of the cracked components of the structures and searches for different detection strategies to diagnose fracture damage. Dimarogonas (2) provided a comprehensive literature review of the vibration of cracked structures and cites more than 300 papers. The review is divided into several sections; methods that describe local flexibility due to cracks, nonlinearities introduced into the system, and local stiffness matrix descriptions of the cracked section. The crack leads to a coupled system that can be recognized from additional harmonics in the frequency spectrum. The subharmonic resonances at approximately half and one third of the bending critical speed of the rotor are reported to be the prominent crack indicators by Gasch $(3,4)$ and Chan $(5)$. By utilizing a single parameter "hinge" crack model, Gasch, provided an overview of the dynamic behavior of a simple rotor with transverse crack. He assumed weight dominance and employed a perturbation method into his analysis. Cross-coupling stiffness and dynamic response terms were not included in his analysis. Mayes model (6) is more practical for deep cracks than a hinged model. Based on Mayes modified model, Sawicki and Wu et al. $(7,8)$ studied the transient vibration response of a cracked Jeffcott rotor under constant acceleration ratios and under constant external torque. The angle between the crack centerline and the rotor whirl vector is employed to determine the closing and opening of the crack. This allows one study of the rotor dynamic 
response with or without the rotor weight dominance assumption by taking non-synchronous whirl into account. Sawicki and Wu et al.(9) investigated the nonlinear dynamic response of a cracked one-mass Jeffcott rotor by means of bifurcation plots. When a rotor with the crack depth of 0.4 spins at some speed ranges, both the lateral and torsional vibration responses sustain periodic, quasi-periodic or chaotic behavior. Muszynska et al. (10) and Bently et al.(11) discuss rotor coupled lateral and torsional vibrations due to unbalance, as well as due to shaft anisotropy under a constant radial preload force. Their experimental results exhibited the existence of significant torsional vibrations due to coupling with the lateral modes. In Bently and Muszynska's experiments, an asymmetric shaft was used to simulate the behavior of a crack.

The current paper extends the research investigation of Bently et al. (11) by using the simpler anisotropic shaft model to characterize a cracked shaft and employing the methods used by Wu et al. (12) to develop a vibration model. Using energy equations a four degrees of freedom analytical model is developed for a two-disk rotor with shaft asymmetry. A radial constant force is applied to the outboard disk to emphasize the effects of gravity or aerodynamic side loading. As load and stiffness asymmetry increase, the vibration amplitudes in both lateral and torsional directions increase, resulting in torsional vibration with amplitudes that may be measured in practical applications. This paper places special emphasis on characterizing the torsional vibration trends associated with shaft asymmetry which may be used to identify failing shafts in operational rotor systems. 


\section{Chapter II Rotor Model}

\section{II.1 Definition of System}

The system shown in Figure 1 represents a practical rotor system. An electric motor drives a shaft through a flexible coupling. The shaft is supported by relatively rigid bearings that in turn support two rotors. The inboard rotor is spanned closely by bearings while the outboard rotor is supported by a wide bearing span. The outboard rotor is subject to external lateral side loads.

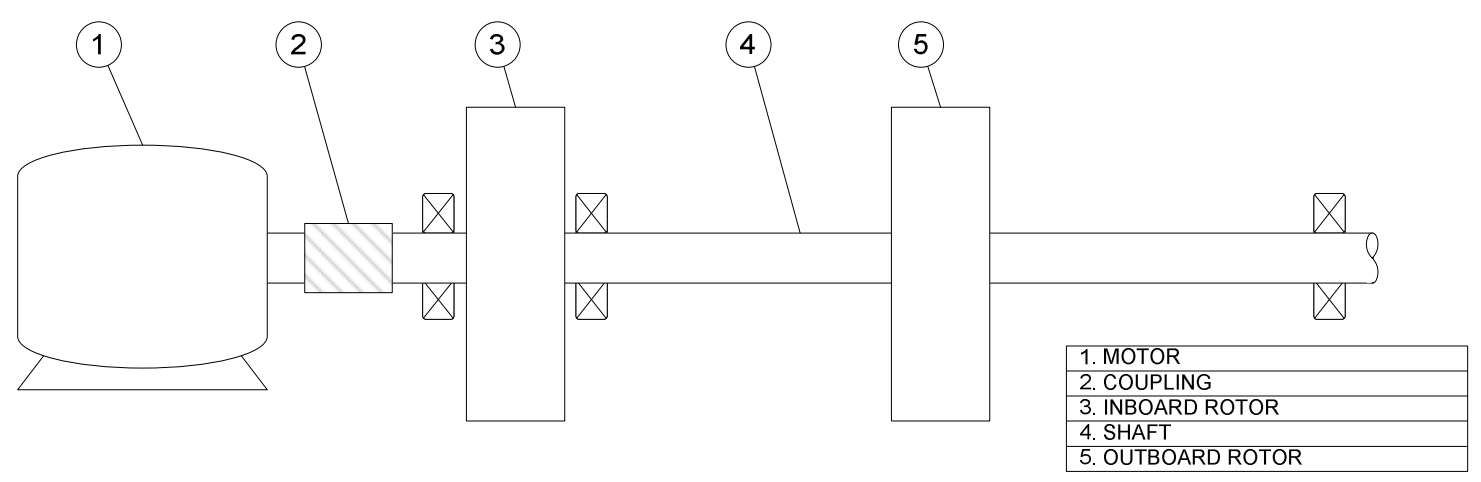

Figure 1: Rotor system

This system is analogous to a modern rotating machine consisting of a turbine driving a compressor. In this case the inboard rotor and motor represent the driving torque and rotational inertia of a turbine. The outboard rotor represents a compressor. Side loading at the outboard rotor might represent the side loading a compressor would undertake due to gravity, aerodynamic loading, or gearbox reactions. 


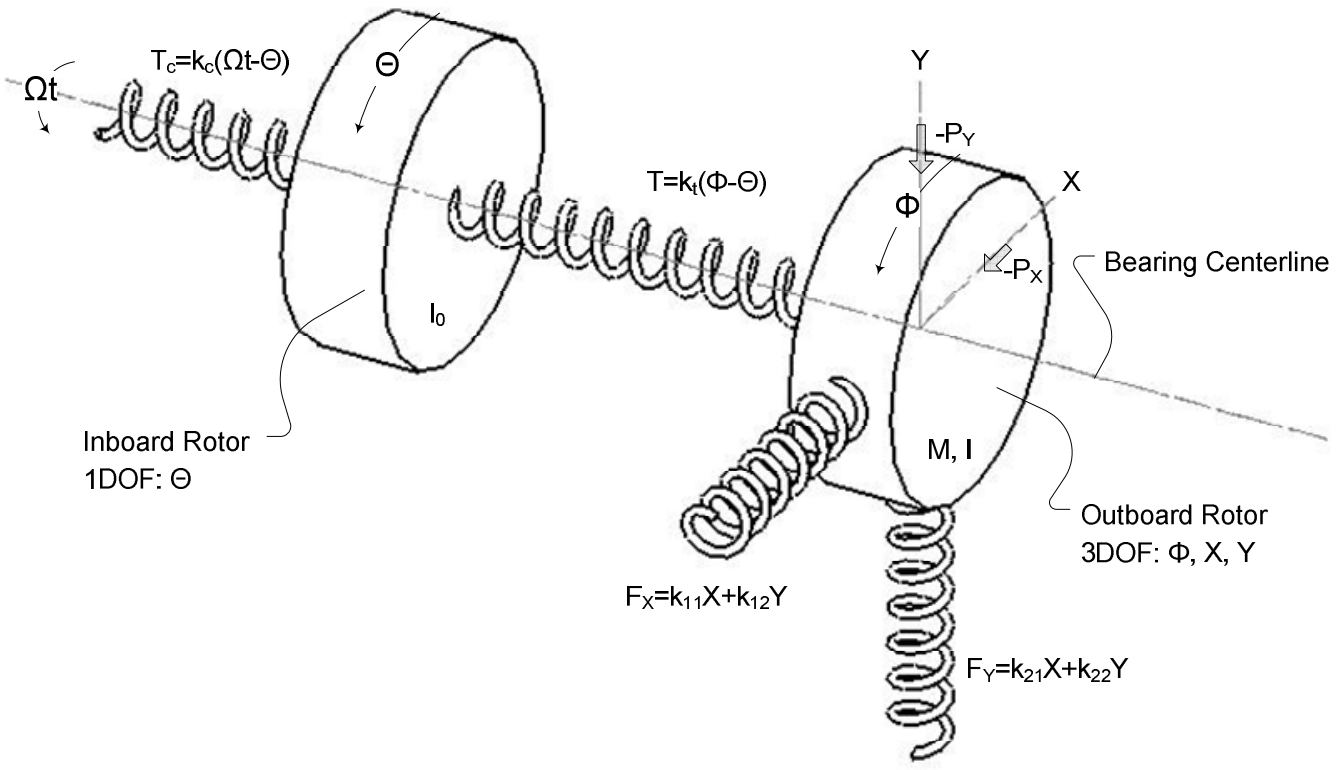

Figure 2: Rotor system model (damping not shown)

Figure 2 shows a vibration model for the physical system depicted by Figure 1. The motor is modeled as a source of rotational energy providing a constant angular velocity, $\Omega$. The attached coupling acts as both a torsional damper and torsional spring. The shaft is modeled by multiple elements: a torsional spring connecting the two rotors, two lateral springs aligned with a fixed $\mathrm{X}-\mathrm{Y}$ coordinate system, and torsional and lateral dampers (not shown). Because the inboard rotor is held laterally by close-mounted rigid bearings, it is modeled with only one rotational degree of freedom, $\Theta$, and no lateral flexibility. The outboard rotor is allowed to move both rotationally and laterally due to the flexible nature of the shaft, so it is modeled three degrees of freedom, $\mathrm{X}, \mathrm{Y}$ and $\Phi$.

\section{II.2 Lateral Stiffness Model}

For an axis-symmetric shaft, lateral stiffness is conveniently described in the rotating $\xi-\eta$ coordinate system shown in Figure 3. The $\eta$-direction is always aligned with the strong direction of the shaft while the $\xi$-direction is always aligned with the weak direction of the shaft. This unique coordinate system exhibits uncoupled stiffness; loads in the $\eta$-direction produce displacement only in the $\eta$-direction and loads in the $\xi$-direction produce displacements only in the $\xi$-direction. 


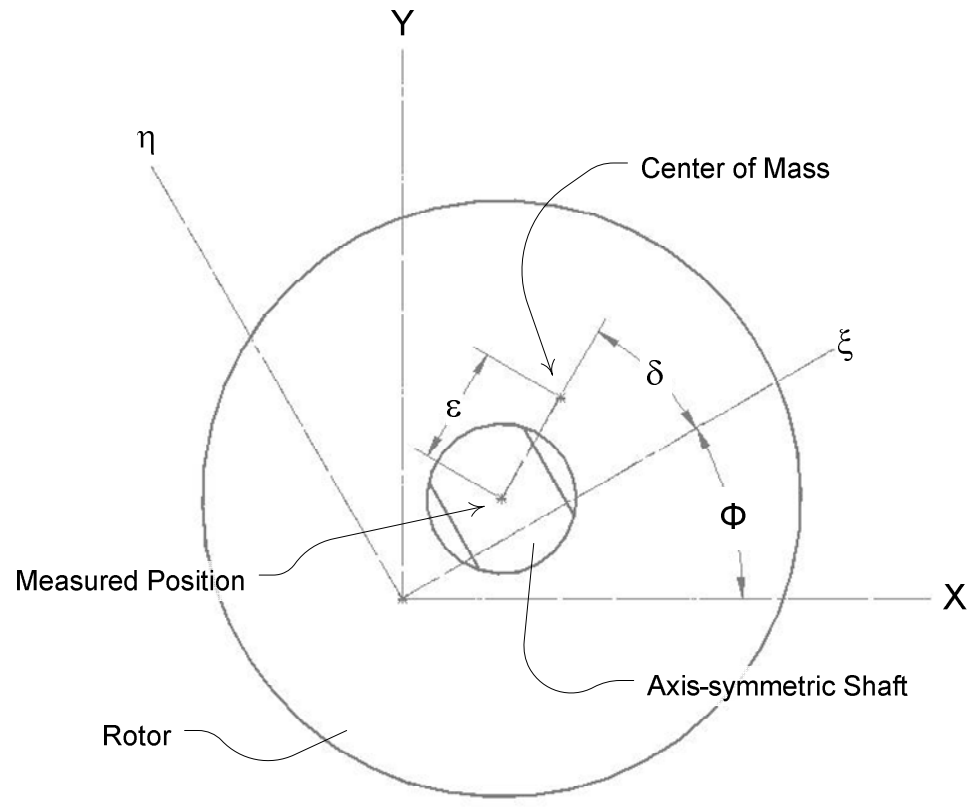

Figure 3: Outboard rotor parameters

In this special coordinate system, forces exerted by the shaft are described by Eq. II-1. Stiffness is defined in the strong and weak directions as $k_{\xi}$ and $k_{\eta}$ respectively. The displacements $\eta$ and $\xi$ are measured from the geometric bearing centerline to the geometric center of the rotor.

$$
\left[\begin{array}{c}
F_{\xi} \\
F_{\eta}
\end{array}\right]=\left[\begin{array}{cc}
k_{\xi} & 0 \\
0 & k_{\eta}
\end{array}\right]\left[\begin{array}{l}
\xi \\
\eta
\end{array}\right]
$$

Eq. II-1

The stiffness matrix in the rotating coordinate system $\left(K_{R}\right)$ is defined by Eq. II-2.

$$
K_{R}=\left[\begin{array}{cc}
k_{\xi} & 0 \\
0 & k_{\eta}
\end{array}\right]
$$

Eq. II-2

In practical applications, it is more convenient to measure displacements in an inertial $\mathrm{X}$-Y coordinate frame. Shaft loads and displacements in the inertial coordinate system are related by the stiffness matrix, $K_{I}$, as shown in Eq. II-3 and Eq. II-4.

$$
\left[\begin{array}{c}
F_{X} \\
F_{Y}
\end{array}\right]=\left[\begin{array}{ll}
k_{11} & k_{12} \\
k_{21} & k_{22}
\end{array}\right]\left[\begin{array}{l}
X \\
Y
\end{array}\right]
$$




$$
K_{I}=\left[\begin{array}{ll}
k_{11} & k_{12} \\
k_{21} & k_{22}
\end{array}\right]
$$

Eq. II-4

To determine values that appropriately populate the inertial stiffness matrix the following coordinate transformation can be made.

$$
K_{I}=T K_{R} T^{-1}
$$

Eq. II-5

where

$$
T=\left[\begin{array}{cc}
\cos \Phi & -\sin \Phi \\
\sin \Phi & \cos \Phi
\end{array}\right]
$$

Eq. II-6

Defining the average stiffness $k=\frac{k_{\xi}+k_{\eta}}{2}$ and the shaft asymmetry factor $q=\frac{k_{\eta}-k_{\xi}}{2 k}$, Eq. II-5produces the inertial stiffness matrix shown in Eq. II-7.

$$
K_{I}=k\left[\begin{array}{cc}
1-q \cos 2 \Phi & -q \sin 2 \Phi \\
-q \sin 2 \Phi & 1+q \cos 2 \Phi
\end{array}\right]
$$

Given any rotational position $\Phi$ and lateral displacement $\mathrm{X}$ and $\mathrm{Y}$ of the rotor centerline, the inertial stiffness matrix may be used calculate loads acting on the rotor by the flexible shaft.

\section{II.3 General Equations of Motion}

The equations of motion governing the system shown in Figure 2 can be derived using Lagrange's equations. The general form of Lagrange's equations is shown in Eq. II-8, where $q_{i}$ is a generalized coordinate, $Q_{i}$ is the generalized load(s) in the direction of $q_{i}$ that produce work, $T$ and $U$ are the kinetic and potential energies of the system, $D$ is the dissipation function of the system, and $n$ is the number of degrees of freedom. 


$$
\frac{\partial}{\partial t}\left(\frac{\partial T}{\partial \dot{q}_{i}}\right)-\frac{\partial T}{\partial q_{i}}+\frac{\partial U}{\partial q_{i}}+\frac{\partial D}{\partial \dot{q}_{i}}=Q_{i} \quad i=1, \ldots, n
$$

Eq. $I I-8$

The kinetic energy of the system is described by Eq. II-11below.

$$
T=\underbrace{\frac{1}{2} I \dot{\Phi}^{2}}_{\substack{\text { Outboard dotor } \\ \text { Rotational Kinetic Energy }}}+\underbrace{\frac{1}{I_{0} \dot{\Theta}^{2}}}_{\substack{\text { Inboard Rotor } \\ \text { Rotational Kinetic Energy }}}+\underbrace{\frac{1}{2} M\left\{\dot{X}^{2}+\dot{Y}^{2}+2 \delta \dot{\Phi}[\dot{Y} \cos (\Phi+\delta)-\dot{X} \sin (\Phi+\delta)]+\varepsilon^{2} \dot{\Phi}^{2}\right\}}_{\text {Outboard Rotor Lateral Kinetic Energy }}
$$

Eq. II-9

For a non-specific lateral shaft stiffness having $k_{12}=k_{21}$, the potential energy of the system is

$$
U=\underbrace{\frac{1}{2}\left(k_{11} X^{2}+k_{22} Y^{2}\right)+k_{12} X Y}_{\text {Energy Stored In Shaft Through Lateral Deflection }}+\underbrace{\frac{1}{2} k_{t}(\Phi-\Theta)^{2}}_{\text {Energy Stored in Shaft Through Torsional Deflection }}
$$

Eq. II- 10

For the axis-symmetric shaft, Eq. II-7 and Eq. II-10 produce Eq. II-11.

$$
U=\frac{1}{2} k\left[(1-q \cos 2 \Phi) X^{2}+(1+q \cos 2 \Phi) Y^{2}\right]-k q \sin 2 \Phi X Y+\frac{1}{2} k_{t}(\Phi-\Theta)^{2}
$$

Eq. II-11

The energy dissipation function can be written as follows

$$
D=\frac{1}{2} C \dot{X}^{2}+\frac{1}{2} C \dot{Y}^{2}+\frac{1}{2} C_{t}(\dot{\Phi}-\dot{\Theta})^{2}
$$

Eq. II-12

Lagrange's equations are formulated for this system as follows

$$
\begin{gathered}
\frac{\partial}{\partial t}\left(\frac{\partial T}{\partial \dot{X}}\right)-\frac{\partial T}{\partial X}+\frac{\partial U}{\partial X}+\frac{\partial D}{\partial \dot{X}}=-P_{X} \\
\frac{\partial}{\partial t}\left(\frac{\partial T}{\partial \dot{Y}}\right)-\frac{\partial T}{\partial Y}+\frac{\partial U}{\partial Y}+\frac{\partial D}{\partial \dot{Y}}=-P_{Y} \\
\frac{\partial}{\partial t}\left(\frac{\partial T}{\partial \dot{\Phi}}\right)-\frac{\partial T}{\partial \Phi}+\frac{\partial U}{\partial \Phi}+\frac{\partial D}{\partial \dot{\Phi}}=0 \\
\frac{\partial}{\partial t}\left(\frac{\partial T}{\partial \dot{\Theta}}\right)-\frac{\partial T}{\partial \Theta}+\frac{\partial U}{\partial \Theta}+\frac{\partial D}{\partial \dot{\Theta}}=-T_{D R}
\end{gathered}
$$

Eq. II-13

The solutions for Eq. II-12 using Eq. II-9, Eq. II-11, and Eq. II-12 are as follows. 


$$
\begin{gathered}
\ddot{X}+2 \varsigma \omega_{n} \dot{X}+\omega_{n}^{2}(1-q \cos 2 \Phi) X=\varepsilon \ddot{\Phi} \sin (\Phi+\delta)+\varepsilon \dot{\Phi}^{2} \cos (\Phi+\delta)+\omega_{n}^{2} q \sin 2 \Phi Y-\frac{P_{X}}{M} \\
\ddot{Y}+2 \varsigma \omega_{n} \dot{Y}+\omega_{n}^{2}(1+q \cos 2 \Phi) Y=-\varepsilon \ddot{\Phi} \cos (\Phi+\delta)+\varepsilon \dot{\Phi}^{2} \sin (\Phi+\delta)+\omega_{n}^{2} q \sin 2 \Phi X-\frac{P_{Y}}{M} \\
\ddot{\Phi}+\omega_{t}^{2}(\Phi-\Theta)+2 \varsigma_{t} \omega_{t}(\dot{\Phi}-\dot{\Theta})=\frac{\varepsilon 2 \varsigma \omega_{n}}{\rho^{2}}[\cos (\Phi+\delta) \dot{Y}-\sin (\Phi+\delta) \dot{X}] \\
+\frac{\varepsilon \omega_{n}^{2}}{\rho^{2}}[\cos (\Phi+\delta) Y-\sin (\Phi+\delta) X]+\frac{\varepsilon \omega_{n}^{2} q}{\rho^{2}}[\cos (\Phi-\delta) Y-\sin (\Phi-\delta) X] \\
+\frac{\varepsilon}{\rho^{2}}\left[\left(\frac{P_{Y}}{M}\right) \cos (\Phi+\delta)-\left(\frac{P_{X}}{M}\right) \sin (\Phi+\delta)\right]+\frac{\omega_{n}^{2} q}{\rho^{2}}\left[2 \cos 2 \Phi X Y-\sin 2 \Phi\left(X^{2}-Y^{2}\right)\right] \\
\ddot{\Theta}-\frac{k_{t}}{I_{0}}(\Phi-\Theta)-\frac{C_{t}}{I_{0}}(\dot{\Phi}-\dot{\Theta})=-\frac{T_{D R}}{I_{0}}
\end{gathered}
$$

Eq. II-14

Assuming that the motor will run at a constant speed and the outboard rotor will vibrate about a static lateral offset, it is convenient to express Eq. II-14 in terms of vibration coordinates $X_{M}=X+\frac{P_{X}}{k}$, $Y_{M}=Y+\frac{P_{Y}}{k}, \varphi=\Phi-\Omega t$, and $\theta=\Theta-\Omega t$. Additionally, the motor drive torque can be expressed as a function of the inboard rotor rotational vibration $\theta$ and coupling properties $C_{R}=C_{c} / C_{t}$ and $K_{R}=k_{c} / k_{t}$. Substitution of these terms into Eq. II-14 produces Eq. II-15 below.

$$
\begin{aligned}
& \ddot{X}_{M}+2 \varsigma \omega_{n} \dot{X}_{M}+\omega_{n}^{2}(1-q \cos (2(\Omega t+\varphi))) X_{M}-\omega_{n}^{2} q \sin (2(\Omega t+\varphi)) Y_{M}=\varepsilon \ddot{\varphi} \sin (\Omega t+\varphi+\delta) \\
& +\varepsilon(\Omega+\dot{\varphi})^{2} \cos (\Omega t+\varphi+\delta)-\left(\frac{P_{Y}}{M}\right) q \sin (2(\Omega t+\varphi))-\left(\frac{P_{X}}{M}\right) q \cos (2(\Omega t+\varphi)) \\
& \ddot{Y}_{M}+2 \varsigma \omega_{n} \dot{Y}_{M}+\omega_{n}^{2}(1+q \cos (2(\Omega t+\varphi))) Y_{M}-\omega_{n}^{2} q \sin (2(\Omega t+\varphi)) X_{M}=-\varepsilon \ddot{\varphi} \cos (\Omega t+\varphi+\delta) \\
& +\varepsilon(\Omega+\dot{\varphi})^{2} \sin (\Omega t+\varphi+\delta)-\left(\frac{P_{X}}{M}\right) q \sin (2(\Omega t+\varphi))+\left(\frac{P_{Y}}{M}\right) q \cos (2(\Omega t+\varphi)) \\
& \ddot{\varphi}+\omega_{t}^{2}(\varphi-\theta)+2 \varsigma_{t} \omega_{t}(\dot{\varphi}-\dot{\theta})=-\frac{\varepsilon q}{\rho^{2}}\left[\left(\frac{P_{Y}}{M}\right) \cos (\Omega t+\varphi-\delta)-\left(\frac{P_{X}}{M}\right) \sin (\Omega t+\varphi-\delta)\right] \\
& +\frac{q}{\omega_{n}^{2} \rho^{2}}\left[2 \cos (2(\Omega t+\varphi))\left(\frac{P_{Y}}{M}\right)\left(\frac{P_{X}}{M}\right)-\sin (2(\Omega t+\varphi))\left(\frac{P_{X}}{M}\right)^{2}+\sin (2(\Omega t+\varphi))\left(\frac{P_{Y}}{M}\right)^{2}\right]+\Gamma
\end{aligned}
$$




$$
\begin{aligned}
& \Gamma=\frac{\varepsilon 2 \varsigma \omega_{n}}{\rho^{2}}\left[\cos (\Omega t+\varphi+\delta) \dot{Y}_{M}-\sin (\Omega t+\varphi+\delta) \dot{X}_{M}\right]+\frac{\varepsilon \omega_{n}^{2}}{\rho^{2}}\left[\cos (\Omega t+\varphi+\delta) Y_{M}-\sin (\Omega t+\varphi+\delta) X_{M}\right] \\
& +\frac{\varepsilon \omega_{n}^{2} q}{\rho^{2}}\left[\cos (\Omega t+\varphi-\delta) Y_{M}-\sin (\Omega t+\varphi-\delta) X_{M}\right] \\
& +\frac{2 q}{\rho^{2}}\left[\cos (2(\Omega t+\varphi))\left(-\left(\frac{P_{Y}}{M}\right) X_{M}-\left(\frac{P_{X}}{M}\right) Y_{M}\right)-\sin (2(\Omega t+\varphi))\left(\left(\frac{P_{Y}}{M}\right) Y_{M}-\left(\frac{P_{X}}{M}\right) X_{M}\right)\right] \\
& +\frac{\omega_{n}^{2} q}{\rho^{2}}\left[2 \cos (2(\Omega t+\varphi)) X_{M} Y_{M}-\sin (2(\Omega t+\varphi)) X_{M}^{2}+\sin (2(\Omega t+\varphi)) Y_{M}^{2}\right] \\
& \ddot{\theta}+\left(1+C_{R}\right) 2 \varsigma_{t} \omega_{t} \dot{\theta}+\left(1+K_{R}\right) \omega_{t}^{2} \theta=\omega_{t}^{2} \varphi+2 \varsigma_{t} \omega_{t} \dot{\varphi}
\end{aligned}
$$

Eq. II- 15

Non-dimensional time $\tau=\omega_{n} t$ may be introduced for modeling purposes. The chain rule allows simple introduction of $\tau$ Eq. II-15 since $\frac{d(\square)}{d t}=\omega_{n} \frac{d(\square)}{d \tau}$ and $\frac{d^{2}(\square)}{d t^{2}}=\omega_{n}^{2} \frac{d^{2}(\square)}{d \tau^{2}}{ }^{1}$. The result is Eq. II-16 below.

$$
\begin{gathered}
X_{M}^{\prime \prime}+2 \varsigma X_{M}^{\prime}+\left(1-q \cos \left(2\left(\frac{\Omega}{\omega_{n}} \tau+\varphi\right)\right)\right) X_{M}-q \sin \left(2\left(\frac{\Omega}{\omega_{n}} \tau+\varphi\right)\right) Y_{M}=\varepsilon \varphi^{\prime \prime} \sin \left(\frac{\Omega}{\omega_{n}} \tau+\varphi+\delta\right) \\
+\frac{\varepsilon}{\omega_{n}^{2}}\left(\Omega+\omega_{n} \varphi^{\prime}\right)^{2} \cos \left(\frac{\Omega}{\omega_{n}} \tau+\varphi+\delta\right)-\left(\frac{P_{Y}}{M}\right) \frac{q}{\omega_{n}^{2}} \sin \left(2\left(\frac{\Omega}{\omega_{n}} \tau+\varphi\right)\right)-\left(\frac{P_{X}}{M}\right) \frac{q}{\omega_{n}^{2}} \cos \left(2\left(\frac{\Omega}{\omega_{n}} \tau+\varphi\right)\right) \\
Y_{M}^{\prime \prime}+2 \varsigma Y_{M}^{\prime}+\left(1+q \cos \left(2\left(\frac{\Omega}{\omega_{n}} \tau+\varphi\right)\right)\right) Y_{M}-q \sin \left(2\left(\frac{\Omega}{\omega_{n}} \tau+\varphi\right)\right) X_{M}=-\varepsilon \varphi^{\prime \prime} \cos \left(\frac{\Omega}{\omega_{n}} \tau+\varphi+\delta\right) \\
+\frac{\varepsilon}{\omega_{n}^{2}}\left(\Omega+\omega_{n} \varphi^{\prime}\right)^{2} \sin \left(\frac{\Omega}{\omega_{n}} \tau+\varphi+\delta\right)-\left(\frac{P_{X}}{M}\right) \frac{q}{\omega_{n}^{2}} \sin \left(2\left(\frac{\Omega}{\omega_{n}} \tau+\varphi\right)\right)+\left(\frac{P_{Y}}{M}\right) \frac{q}{\omega_{n}^{2}} \cos \left(2\left(\frac{\Omega}{\omega_{n}} \tau+\varphi\right)\right) \\
\varphi^{\prime \prime}+\left(\frac{\omega_{t}}{\omega_{n}}\right)^{2}(\varphi-\theta)+\frac{2 \varsigma_{t} \omega_{t}}{\omega_{n}}\left(\varphi^{\prime}-\theta^{\prime}\right)=-\frac{\varepsilon q}{\omega_{n}^{2} \rho^{2}}\left[\left(\frac{P_{Y}}{M}\right) \cos \left(\frac{\Omega}{\omega_{n}} \tau+\varphi-\delta\right)-\left(\frac{P_{X}}{M}\right) \sin \left(\frac{\Omega}{\omega_{n}} \tau+\varphi-\delta\right)\right] \\
+\frac{q}{\omega_{n}^{4} \rho^{2}}\left[2 \cos \left(2\left(\frac{\Omega}{\omega_{n}} \tau+\varphi\right)\right)\left(\frac{P_{Y}}{M}\right)\left(\frac{P_{X}}{M}\right)-\sin \left(2\left(\frac{\Omega}{\omega_{n}} \tau+\varphi\right)\right)\left(\frac{P_{X}}{M}\right)^{2}+\sin \left(2\left(\frac{\Omega}{\omega_{n}} \tau+\varphi\right)\right)\left(\frac{P_{Y}}{M}\right)^{2}\right]+\Gamma_{n}
\end{gathered}
$$

${ }^{1}$ For notation purposes, non-dimensional time derivatives are expressed as hatch marks (i.e. $(\square)^{\prime}=\frac{d(\square)}{d \tau}$ and $\left.(\square)^{\prime \prime}=\frac{d^{2}(\square)}{d \tau^{2}}\right)$ 


$$
\begin{aligned}
& \Gamma_{n}=\frac{\varepsilon 2 \varsigma}{\rho^{2}}\left[\cos \left(\frac{\Omega}{\omega_{n}} \tau+\varphi+\delta\right) Y_{M}^{\prime}-\sin \left(\frac{\Omega}{\omega_{n}} \tau+\varphi+\delta\right) X_{M}^{\prime}\right]+\frac{\varepsilon}{\rho^{2}}\left[\cos \left(\frac{\Omega}{\omega_{n}} \tau+\varphi+\delta\right) Y_{M}-\sin \left(\frac{\Omega}{\omega_{n}} \tau+\varphi+\delta\right) X_{M}\right] \\
& +\frac{\varepsilon q}{\rho^{2}}\left[\cos \left(\frac{\Omega}{\omega_{n}} \tau+\varphi-\delta\right) Y_{M}-\sin \left(\frac{\Omega}{\omega_{n}} \tau+\varphi-\delta\right) X_{M}\right] \\
& +\frac{2 q}{\omega_{n}^{2} \rho^{2}}\left[\cos \left(2\left(\frac{\Omega}{\omega_{n}} \tau+\varphi\right)\right)\left(-\left(\frac{P_{Y}}{M}\right) X_{M}-\left(\frac{P_{X}}{M}\right) Y_{M}\right)-\sin \left(2\left(\frac{\Omega}{\omega_{n}} \tau+\varphi\right)\right)\left(\left(\frac{P_{Y}}{M}\right) Y_{M}-\left(\frac{P_{X}}{M}\right) X_{M}\right)\right] \\
& +\frac{q}{\rho^{2}}\left[2 \cos \left(2\left(\frac{\Omega}{\omega_{n}} \tau+\varphi\right)\right) X_{M} Y_{M}-\sin \left(2\left(\frac{\Omega}{\omega_{n}} \tau+\varphi\right)\right) X_{M}^{2}+\sin \left(2\left(\frac{\Omega}{\omega_{n}} \tau+\varphi\right)\right) Y_{M}^{2}\right] \\
& \theta^{\prime \prime}+\left(1+C_{R}\right) 2 \varsigma_{t} \frac{\omega_{t}}{\omega_{n}} \theta^{\prime}+\left(1+K_{R}\right)\left(\frac{\omega_{t}}{\omega_{n}}\right)^{2} \theta=\left(\frac{\omega_{t}}{\omega_{n}}\right)^{2} \varphi+2 \varsigma_{t} \frac{\omega_{t}}{\omega_{n}} \varphi^{\prime}
\end{aligned}
$$

Eq. II-16

While Eq. II-16 provides a complete mathematical description for the system described in section II.1, the physical interpretation is unclear. In sections II.4 and II.5, special cases are explored to develop a better understanding of the system.

\section{II.4 Special Case: Lateral Vibration Only}

For negligible levels of torsional activity, some simplifications can be made to the general equations of motion. This might be the case when operating at a speed that is not near a torsional resonance. Assuming a torsionally rigid system, all elements of the rotor move at a constant rotational velocity $\Omega$ such that $\varphi=0$ and $\theta=0$. Note that the inboard rotor has no vibration influence in this simplified model. For a vertical side load only $\left(P_{X}=0\right)$ and $\delta=0$, Eq. II-16 simplifies to

$$
\begin{gathered}
\ddot{X}_{M}+2 \varsigma \omega_{n} \dot{X}_{M}+\omega_{n}^{2}(1-q \cos (2 \Omega t)) X_{M}=\Omega^{2} \cos (\Omega t)-\left(\frac{P_{Y}}{M}\right) q \sin (2 \Omega t)+\omega_{n}^{2} q \sin (2 \Omega t) Y_{M} \\
\ddot{Y}_{M}+2 \varsigma \omega_{n} \dot{Y}_{M}+\omega_{n}^{2}(1+q \cos (2 \Omega t)) Y_{M}=\Omega^{2} \sin (\Omega t)+\left(\frac{P_{Y}}{M}\right) q \cos (2 \Omega t)+\omega_{n}^{2} q \sin (2 \Omega t) X_{M}
\end{gathered}
$$

Eq. II-17

$1 \mathrm{X}$ (once per motor revolution) and $2 \mathrm{X}$ (twice per motor revolution) lateral forcing functions are clearly shown as functions of the unbalance and side loading respectively. $2 \mathrm{X}$ variations in stiffness are also present as well as $2 \mathrm{X}$ coupling terms that describe the interaction between the two lateral directions. It 
should be noted that all $2 \mathrm{X}$ components are related to the shaft asymmetry, q. If no asymmetry were present, q would become zero and Eq. II-17 would reduce to the description of a Jeffcott rotor with a $1 \mathrm{X}$ forcing term due to unbalance.

\section{II.5 Special Case: Torsional Vibration Only}

When the rotor is operating at a speed that does not excite significant lateral activity, some simplifications can be made to the general equations of motion. Side loading produces a static deflection, however the outboard disk remains in a constant lateral position such that $X_{M}=0$ and $Y_{M}=0$. For rigid coupling $\theta=0$. Assuming only a vertical side load $\left(P_{X}=0\right)$ and $\delta=0$, Eq. II-16 reduces to

$$
\ddot{\varphi}+2 \varsigma_{t} \omega_{t} \dot{\varphi}+\omega_{t}^{2} \varphi=\underbrace{-\frac{\varepsilon q}{\rho^{2}}\left(\frac{P_{Y}}{M}\right) \cos (\Omega t+\varphi)}_{\text {1X Forcing Term }}+\underbrace{\frac{q}{\omega_{n}^{2} \rho^{2}}\left(\frac{P_{Y}}{M}\right)^{2} \sin (2(\Omega t+\varphi))}_{\text {2X Forcing Term }}
$$

Eq. II-18 resembles a linear oscillator with two forcing terms. The $1 \mathrm{X}$ forcing term is linearly proportional to the side load and unbalance eccentricity. The $2 \mathrm{X}$ forcing term is not dependent on the unbalance eccentricity, but has a quadratic dependency on the side load. Manipulation of Eq. II-18 reveals a physically significant relationship.

$$
I \ddot{\varphi}+C_{t} \dot{\varphi}+k_{t} \varphi=-q P_{Y} \varepsilon \cos (\Omega t+\varphi)-q P_{Y} Y \sin (2(\Omega t+\varphi))
$$

Eq. II-19 shows that the $2 \mathrm{X}$ torque amplitude is proportional to the product of the side load $\left(P_{Y}\right)$ and the static displacement due to the side load $\left(Y=-P_{Y} / k\right)$. This highlights an interesting phenomena: a torque that is proportional to $Y$ results from the load $P_{Y}$ acting in the direction of $Y$. Furthermore, the amplitudes of the $1 \mathrm{X}$ and $2 \mathrm{X}$ torsional excitations are proportional to the ratio of unbalance eccentricity to the static displacement due to side loading respectively.

To predict the conditions under which the $1 \mathrm{X}$ and $2 \mathrm{X}$ torsional excitations would be equal in magnitude, the terms are equated: 


$$
\begin{aligned}
\frac{\varepsilon q}{\rho^{2}}\left(\frac{P_{Y}}{M}\right) & =\frac{q}{\omega_{n}^{2} \rho^{2}}\left(\frac{P_{Y}}{M}\right)^{2} \\
\varepsilon & =\frac{P_{Y}}{\omega_{n}^{2} M} \\
\varepsilon & =\frac{P_{Y}}{k}
\end{aligned}
$$

Eq. II-20

Eq. II-20 predicts that the magnitudes of the $1 \mathrm{X}$ and $2 \mathrm{X}$ torsional excitations would be equal when the magnitudes of the eccentricity and average displacement from side loading $\left(\frac{P_{Y}}{k}\right)$ are equal. 


\section{Chapter III Simulation}

\section{III.1 Special Case: Lateral Vibration Only}

For the special case where torsional vibration is considered negligible, $\varphi=0$ and $\theta=0$. If we consider only a vertical load on the outboard rotor $\left(P_{X}=0\right)$, assume no initial displacements $\left(\Phi_{0}=0, \Theta_{0}=0\right)$, and consider the case where unbalance is aligned with the weak axis ( $\delta=0$ ), the equations of motion reduce to Eq. II-17. Non-dimensional time $\tau=\omega_{n} t$ may be introduced for modeling purposes. The chain rule allows simple introduction of $\tau$ into Eq. II-17 since $\frac{d(\square)}{d t}=\omega_{n} \frac{d(\square)}{d \tau}$ and $\frac{d^{2}(\square)}{d t^{2}}=\omega_{n}^{2} \frac{d^{2}(\square)}{d \tau^{2}}$. The result is Eq.

III-1 below.

$$
\begin{aligned}
& X_{M}^{\prime \prime}+2 \varsigma X_{M}^{\prime}+\left(1-q \cos \left(2 \frac{\Omega}{\omega_{n}} \tau\right)\right) X_{M}-q \sin \left(2 \frac{\Omega}{\omega_{n}} \tau\right) Y_{M}=\frac{\varepsilon}{\omega_{n}^{2}} \Omega^{2} \cos \left(\frac{\Omega}{\omega_{n}} \tau\right)-\left(\frac{P_{Y}}{M}\right) \frac{q}{\omega_{n}^{2}} \sin \left(2 \frac{\Omega}{\omega_{n}} \tau\right) \\
& Y_{M}^{\prime \prime}+2 \varsigma Y_{M}^{\prime}+\left(1+q \cos \left(2 \frac{\Omega}{\omega_{n}} \tau\right)\right) Y_{M}-q \sin \left(2 \frac{\Omega}{\omega_{n}} \tau\right) X_{M}=\frac{\varepsilon}{\omega_{n}^{2}} \Omega^{2} \sin \left(\frac{\Omega}{\omega_{n}} \tau\right)+\left(\frac{P_{Y}}{M}\right) \frac{q}{\omega_{n}^{2}} \cos \left(2 \frac{\Omega}{\omega_{n}} \tau\right)
\end{aligned}
$$

Eq. III-1

For the purpose of numerical modeling, let the states $u_{3}=X_{M}, u_{4}=X_{M}^{\prime}, u_{5}=Y_{M}, u_{6}=Y_{M}^{\prime}$. This generates the system of equations described by Eq. III-2.

$$
\begin{gathered}
u_{3}^{\prime}=u_{4} \\
u_{4}^{\prime}=-2 \varsigma u_{4}-\left(1-q \cos \left(2 \frac{\Omega}{\omega_{n}} \tau\right)\right) u_{3}+q \sin \left(2 \frac{\Omega}{\omega_{n}} \tau\right) u_{5}+\frac{\varepsilon}{\omega_{n}^{2}} \Omega^{2} \cos \left(\frac{\Omega}{\omega_{n}} \tau\right)-\left(\frac{P_{Y}}{M}\right) \frac{q}{\omega_{n}^{2}} \sin \left(2 \frac{\Omega}{\omega_{n}} \tau\right) \\
u_{5}^{\prime}=u_{6} \\
u_{6}^{\prime}=-2 \varsigma u_{6}-\left(1+q \cos \left(2 \frac{\Omega}{\omega_{n}} \tau\right)\right) u_{5}+q \sin \left(2 \frac{\Omega}{\omega_{n}} \tau\right) u_{3}+\frac{\varepsilon}{\omega_{n}^{2}} \Omega^{2} \sin \left(\frac{\Omega}{\omega_{n}} \tau\right)+\left(\frac{P_{Y}}{M}\right) \frac{q}{\omega_{n}^{2}} \cos \left(2 \frac{\Omega}{\omega_{n}} \tau\right)
\end{gathered}
$$

Eq. III-2

The solution to this system of equations can be found using computer software, in this case Matlab version 7.1. The function "ode 45 " is used to solve this system of equations. This solver integrates the series of 
differential equations and is based on an explicit version of the Runge-Kutta formula, the Dormand-Prince pair. It is a one-step method that uses initial conditions and solves for conditions at the next time step. Matlab programming for this system can be found in the C.1. Using the parameters listed in Table 1, solutions to Eq. III-2 are generated for a range of rotor speeds. The resulting steady state vibration $\mathrm{X}_{\mathrm{M}}$ and $\mathrm{Y}_{\mathrm{M}}$ amplitudes are plotted in Figure 4.

Table 1: Parameters used for lateral vibration model

\begin{tabular}{|l|l|l|l|l|l|l|}
\hline$\omega_{n}$ & $\varsigma$ & $\varsigma_{t}$ & $\varepsilon$ & $\rho$ & $P y / M$ & $q$ \\
\hline 3500rpm & 0.2 & 0.05 & $0.005 \mathrm{in}$ & 1.0in & 2700in/sec ${ }^{2}$ & 0.15 \\
\hline
\end{tabular}

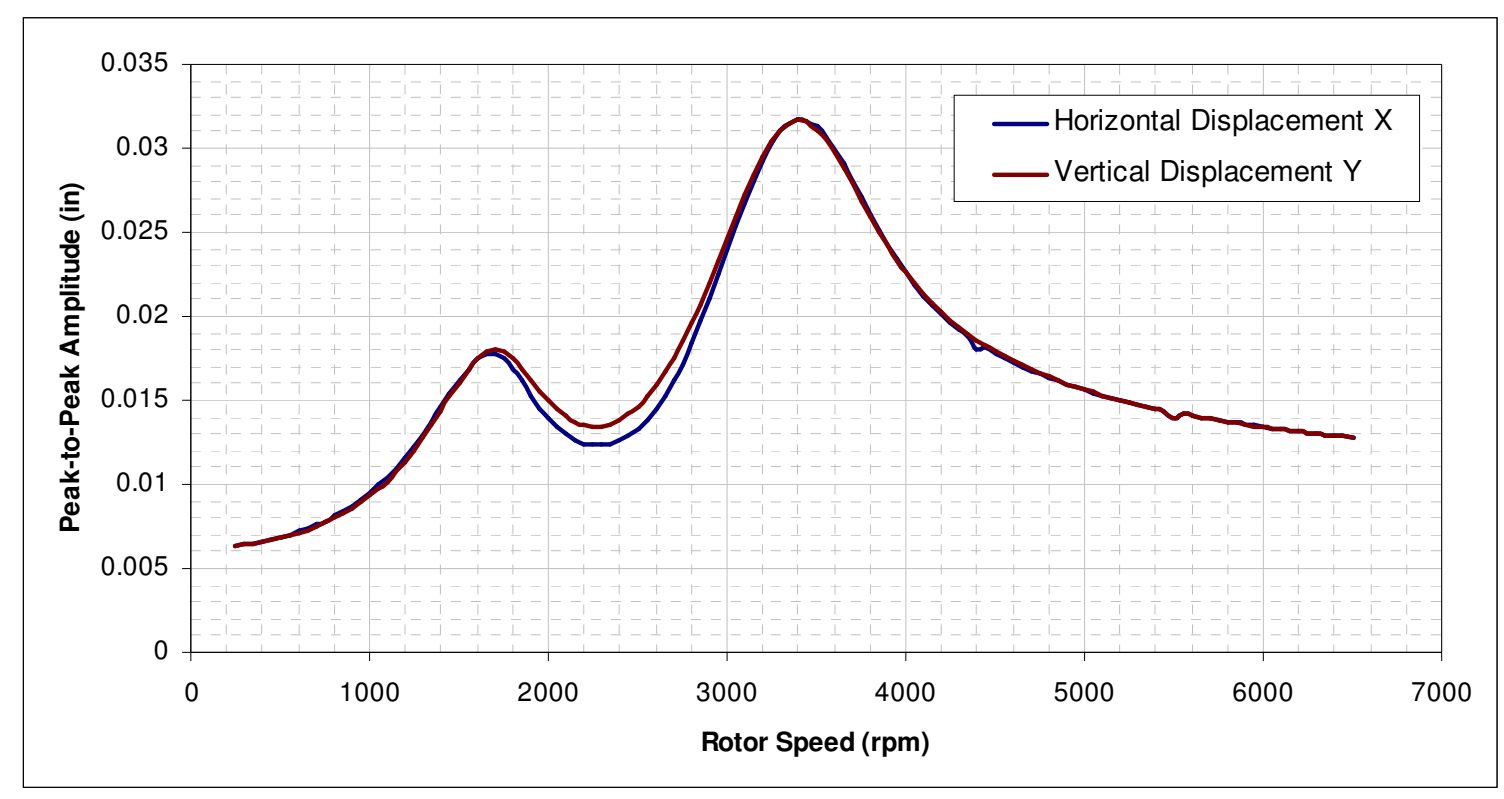

Figure 4: Lateral vibration amplitudes predicted by model

Figure 4 shows amplitude peaks at the natural frequency $\left(\omega_{n}\right)$ and half the natural frequency $\left(\frac{1}{2} \omega_{n}\right)$ as expected. These peaks are associated with the $1 \mathrm{X}$ unbalance excitation and $2 \mathrm{X}$ asymmetry and side load excitations respectively.

In Figure 5 and Figure 6 the orbit response of the rotor is plotted at the $\frac{1}{2} \omega_{n}$ and $\omega_{n}$ rotor speeds. 


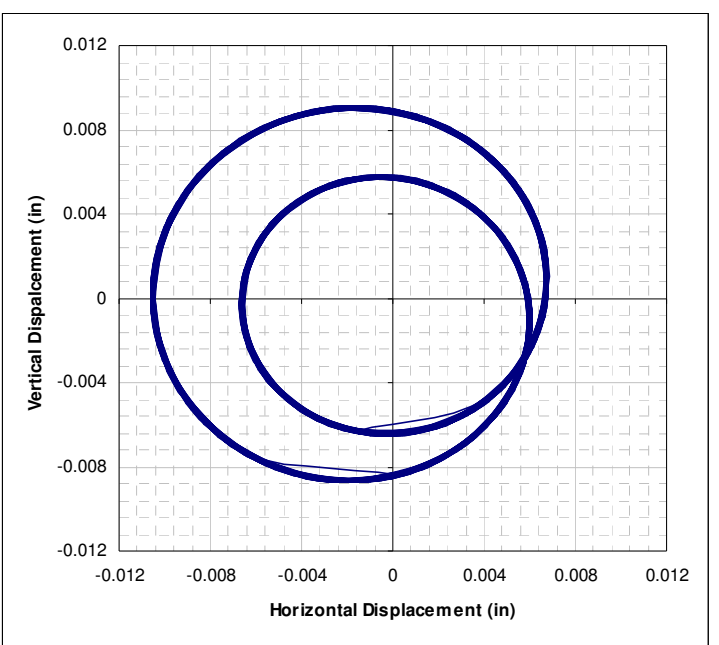

Figure 5: Orbit response at half the lateral

natural frequency $\left(\Omega=\frac{1}{2} \omega_{n}=1750 \mathrm{rpm}\right)$

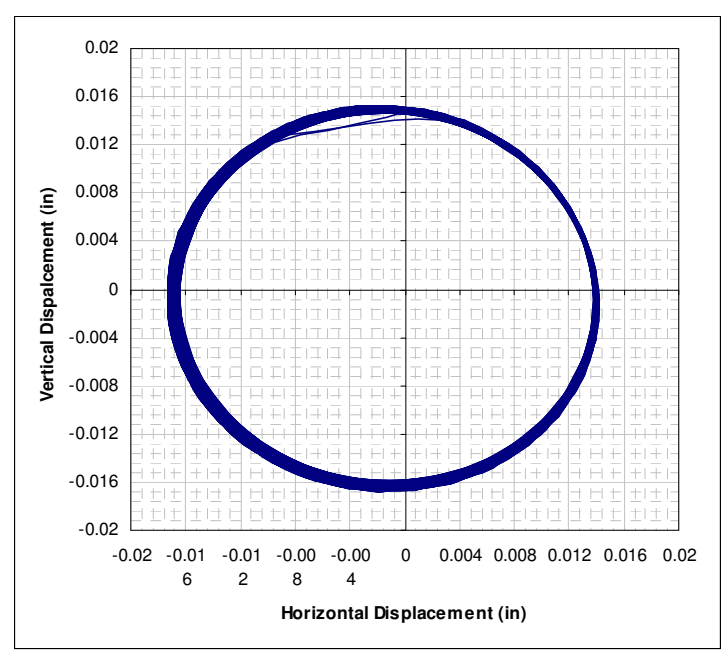

Figure 6: Orbit response at the lateral natural

frequency $\left(\Omega=\omega_{n}=3500 \mathrm{rpm}\right)$

\section{III.2 Special Case: Torsional Vibration Only}

When the rotor operates at a speed that does not excite significant lateral activity, some assumptions and simplifications can be made to the general equations of motion. It is assumed that side loading produces a static deflection, however the outboard disk remains in a relatively constant lateral position such that $X_{M}=0$ and $Y_{M}=0$. For rigid coupling $\theta=0$. Assuming only a vertical side load $\left(P_{X}=0\right)$ and $\delta=0$, the equations of motion simplify to:

$$
\varphi^{\prime \prime}+\left(\frac{\omega_{t}}{\omega_{n}}\right)^{2} \varphi+\frac{2 \varsigma_{t} \omega_{t}}{\omega_{n}} \varphi^{\prime}=-\frac{\varepsilon q}{\omega_{n}^{2} \rho^{2}}\left(\frac{P_{Y}}{M}\right) \cos \left(\frac{\Omega}{\omega_{n}} \tau+\varphi\right)+\frac{q}{\omega_{n}^{4} \rho^{2}} \sin \left(2\left(\frac{\Omega}{\omega_{n}} \tau+\varphi\right)\right)\left(\frac{P_{Y}}{M}\right)^{2}
$$

Eq. III-3

For the purpose of numerical simulation, let $\tau=\omega_{n} t$ and the states $u_{1}=\varphi$ and $u_{2}=\varphi^{\prime}$. The equations of motion become:

$$
u_{1}^{\prime}=u_{2}
$$




$$
u_{2}^{\prime}=-\left(\frac{\omega_{t}}{\omega_{n}}\right)^{2} u_{1}-\frac{2 \varsigma_{t} \omega_{t}}{\omega_{n}} u_{2}-\frac{\varepsilon q}{\omega_{n}^{2} \rho^{2}}\left(\frac{P_{Y}}{M}\right) \cos \left(\frac{\Omega}{\omega_{n}} \tau+u_{1}\right)+\frac{q}{\omega_{n}^{4} \rho^{2}} \sin \left(2\left(\frac{\Omega}{\omega_{n}} \tau+u_{1}\right)\right)\left(\frac{P_{Y}}{M}\right)^{2}
$$

Eq. III-4

Matlab is used to find a solution to Eq. III-4 (see Appendix C.2). Peak-to-peak torsional vibration amplitude is plotted for the parameters in Table 2 in Figure 7.

Table 2: Parameters used for torsional vibration model

\begin{tabular}{|l|l|l|l|l|l|l|l|l|}
\hline$\omega_{\mathrm{n}}$ & $\omega_{\mathrm{t}}$ & $\varsigma$ & $\varsigma_{\mathrm{t}}$ & $\varepsilon$ & $\rho$ & Py/M & $\mathrm{q}$ & $\frac{P_{Y}}{k} / \varepsilon^{2}$ \\
\hline 10000rpm & $3500 \mathrm{rpm}$ & 0.2 & 0.05 & $0.005 \mathrm{in}$ & $1.0 \mathrm{in}$ & $2700 \mathrm{in} / \mathrm{sec}^{2}$ & 0.15 & 0.49 \\
\hline
\end{tabular}

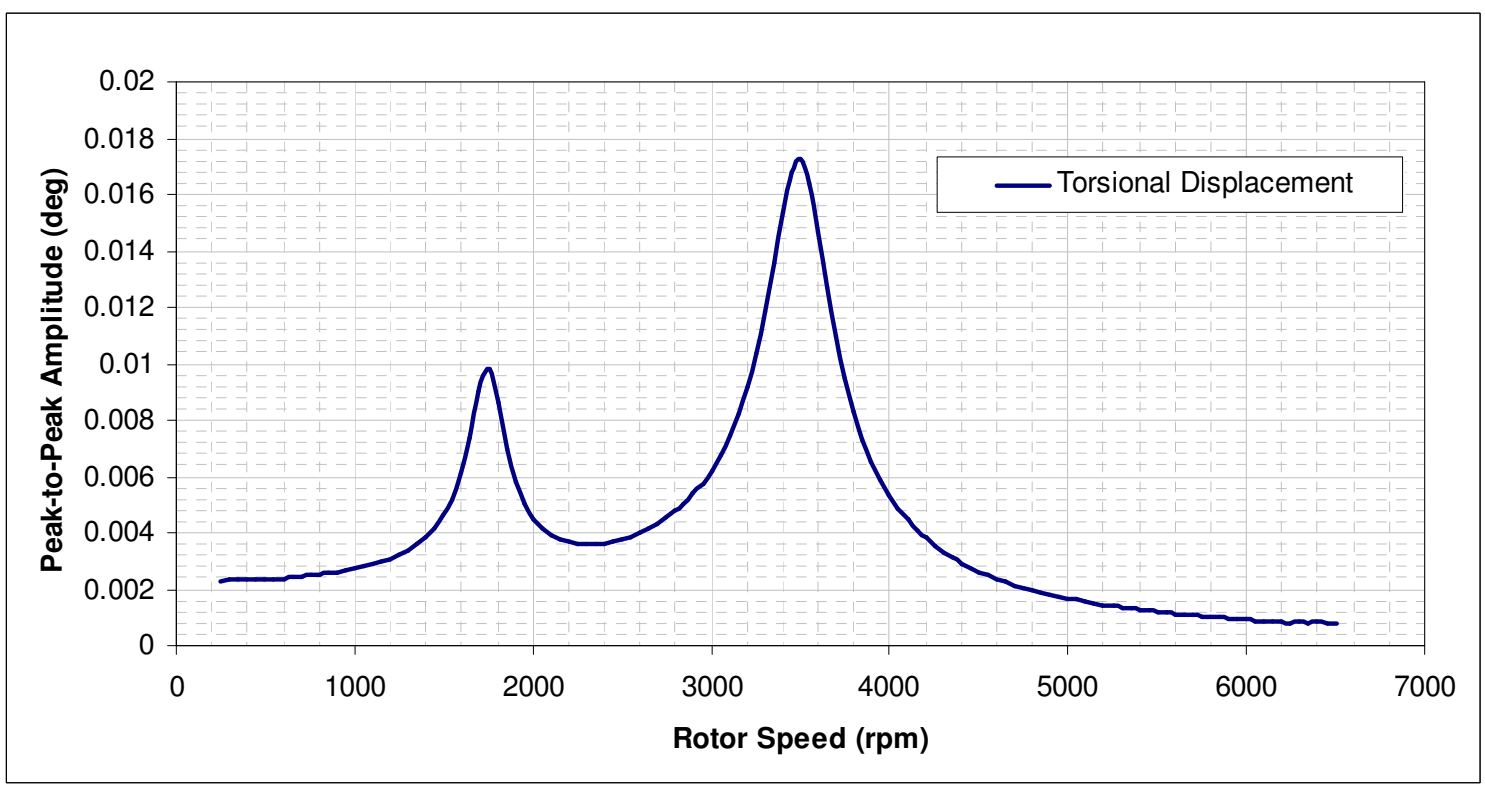

Figure 7: Torsional vibration amplitudes predicted by model

Two peaks are observed corresponding to $\omega_{t}$ and $1 / 2 \omega_{t}$ respectively. It is observed that the ratio of the peak amplitudes at $\omega_{\mathrm{t}}$ and $1 / 2 \omega_{\mathrm{t}}$ approximates the ratio $\frac{P_{Y}}{k} / \varepsilon$. This phenomenon is explored further through parametric study by varying the value of $\frac{P_{Y}}{M}$ and plotting the amplitude and speed against the ratio

${ }^{2}$ Ratio $\frac{P_{Y}}{k} / \varepsilon$ is calculated from parameters $\omega_{\mathrm{n}}$, ee, and Py/M using the relationship $\frac{P_{Y}}{k} / \varepsilon=\frac{P_{Y}}{\omega^{2} M} / e e$ 
$\frac{P_{Y}}{k} / \varepsilon$. The resulting 3D plot using the parameters from Table 2 and increasing values of $\frac{P_{Y}}{M}$ is shown in

Figure 8 below.

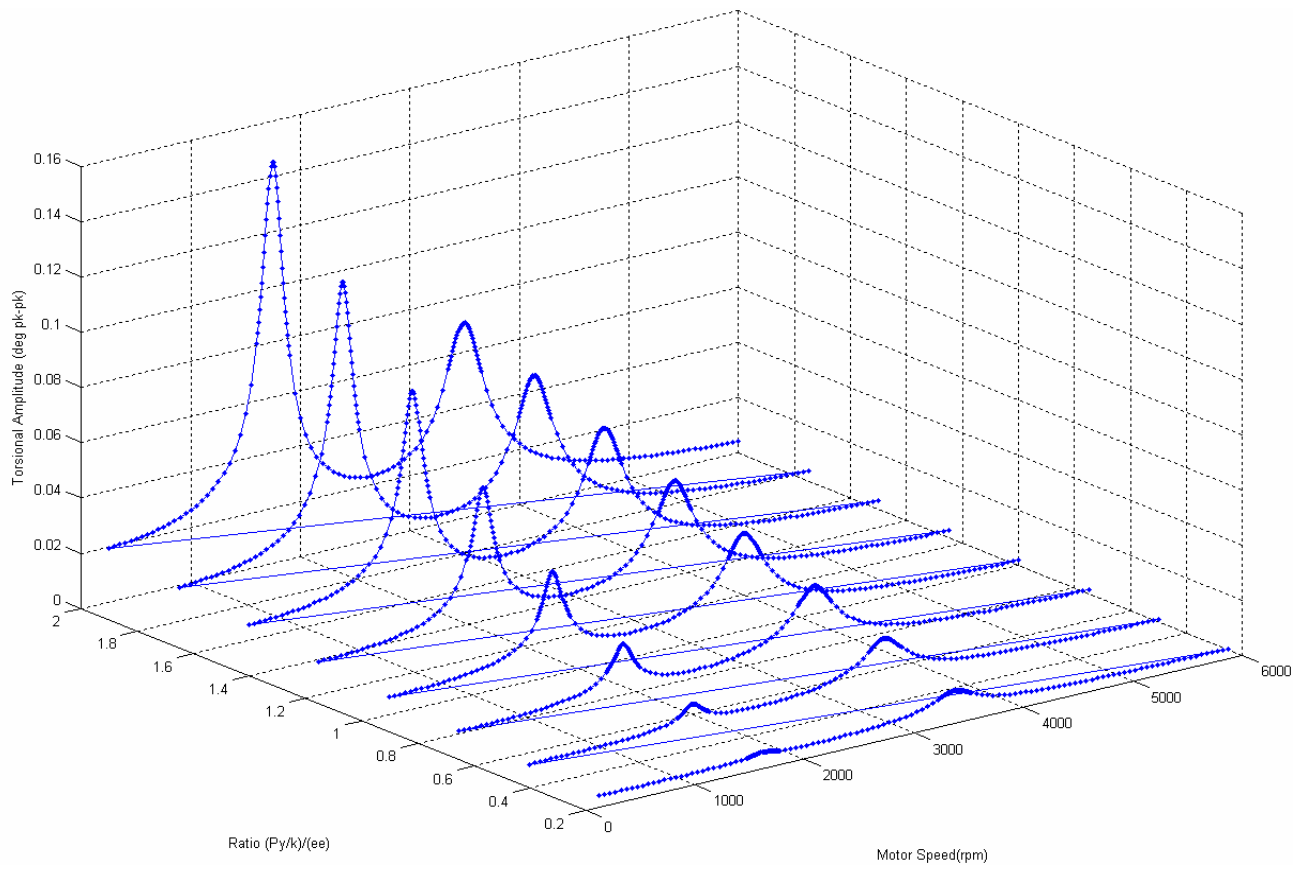

Figure 8: Torsional vibration amplitude vs. speed for varying ratios of $\frac{P_{Y}}{k} / \varepsilon$

It can be seen in Figure 8 that the ratio $\frac{P_{Y}}{k} / \varepsilon$ approximates the ratio of torsional vibration amplitudes at $\omega_{\mathrm{t}}$ and $1 / 2 \omega_{\mathrm{t}}$ (i.e. where $\frac{P_{Y}}{k} / \varepsilon=1$ the torsional vibration amplitudes at $\omega_{\mathrm{t}}$ and $1 / 2 \omega_{\mathrm{t}}$ are approximately equal).

This suggests that torsional vibration associated with a shaft stiffness asymmetry (such as a transverse crack) might be characterized by this ratio. For $\frac{P_{Y}}{k} / \varepsilon<1$ more torsional vibration activity would be expected at speeds near $\omega_{\mathrm{t}}$ and for $\frac{P_{Y}}{k} / \varepsilon>1$ more torsional vibration activity would be expected near $1 / 2 \omega_{\mathrm{t}}$.

Torsional response predicted by the model for the parameters in Table 2 is plotted on a time scale for $1 / 2 \omega_{t}=1750$ rpm and $\omega_{t}=3500$ rpm in Figure 9 and Figure 10 respectively. Near $1 / 2 \omega_{t}$, peaks are predicted every 90 degrees of motor rotation, when the side load is aligned with the strong and weak axes of the shaft. Near $\omega_{t}$ peaks are predicted only when the side load is aligned with the weak axis. 


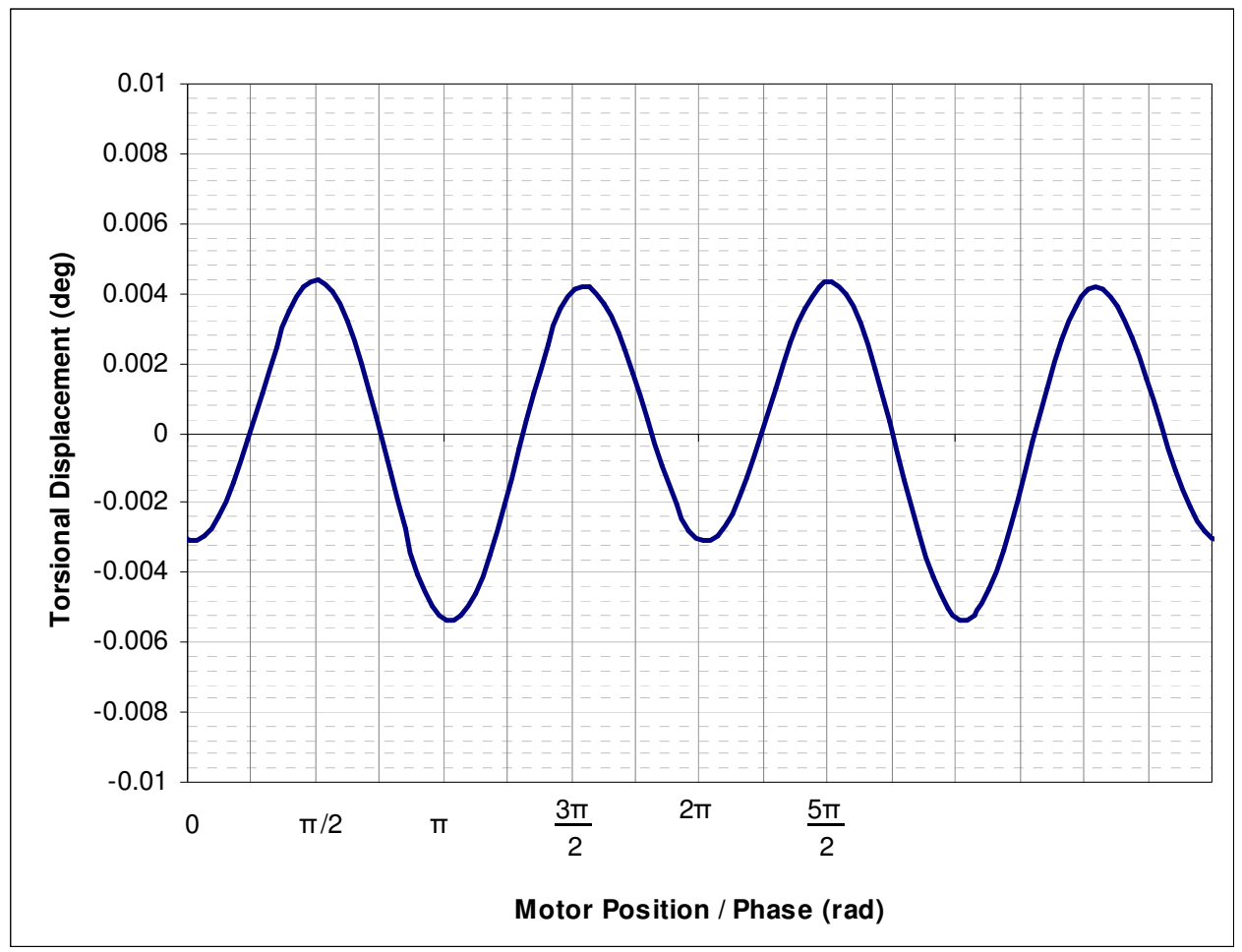

Figure 9: Torsional response at half the torsional natural frequency $\left(\Omega=\frac{1}{2} \omega_{t}=1750 \mathrm{rpm}\right)$

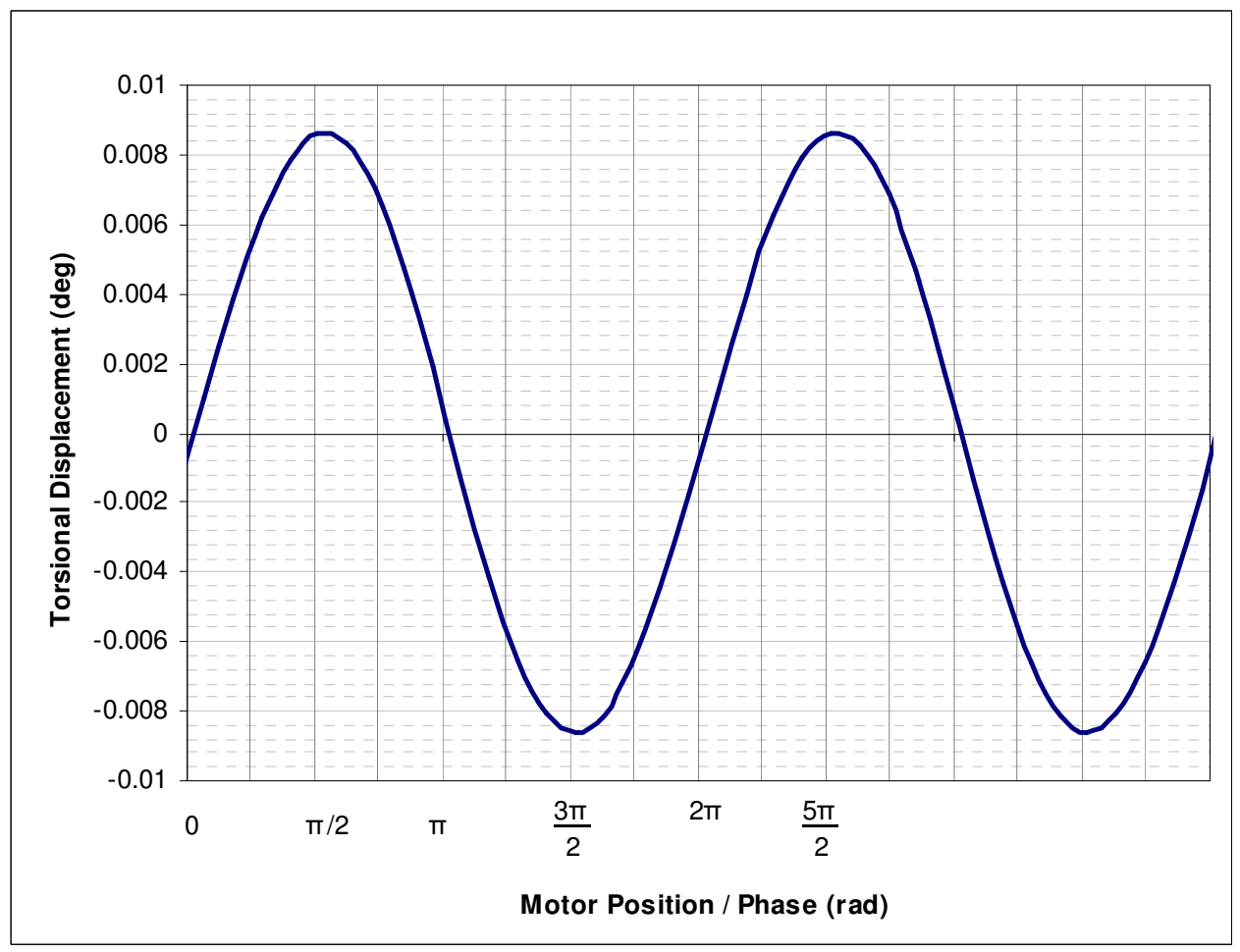

Figure 10: Torsional response at the torsional natural frequency $\left(\Omega=\omega_{t}=3500 \mathrm{rpm}\right)$ 
The case where gravity is the only source of side loading may be of special interest, as might be the case in a horizontal turbine with balanced aerodynamic loading. For the case of a gravitationally side loaded rotor $\mathrm{Py} / \mathrm{M}$ would be the acceleration due to gravity, generally accepted as $32.17 \mathrm{ft} / \mathrm{sec}^{2}$ or $386.1 \mathrm{in} / \mathrm{sec}^{2}$. Using the rotor parameters in Table 3, the Matlab model from Appendix C.2 predicts the response shown in Figure 11. Note the scale of the vertical axis in Figure 11 is the same as in Figure 7 for relative comparison. Response at $1 / 2 \omega_{t}$ is insignificant compared with the response at $\omega_{t}$, as might be expected from the low ratio of $\frac{P_{Y}}{k} / \varepsilon$. Systems with lower lateral natural frequencies may exhibit additional response at $1 / 2 \omega_{\mathrm{t}}$ since the ratio $\frac{P_{Y}}{k} / \varepsilon$ would increase, but a general model that considers both lateral and torsional vibration should be used to investigate such a case; the assumption of negligible lateral activity would be invalidated as $\omega_{n}$ approached $\omega_{\mathrm{t}}$

Table 3: Parameters used for torsional vibration model for gravitational loading

\begin{tabular}{|l|l|l|l|l|l|l|l|l|}
\hline$\omega_{\mathrm{n}}$ & $\omega_{\mathrm{t}}$ & $\varsigma$ & $\varsigma_{\mathrm{t}}$ & $\varepsilon$ & $\rho$ & $\mathrm{Py} / \mathrm{M}$ & $\mathrm{q}$ & $\frac{P_{Y}}{k} / \varepsilon$ \\
\hline $10000 \mathrm{rpm}$ & $3500 \mathrm{rpm}$ & 0.2 & 0.05 & $0.005 \mathrm{in}$ & $1.0 \mathrm{in}$ & $386.1 \mathrm{in} / \mathrm{sec}^{2}$ & 0.15 & 0.07 \\
\hline
\end{tabular}

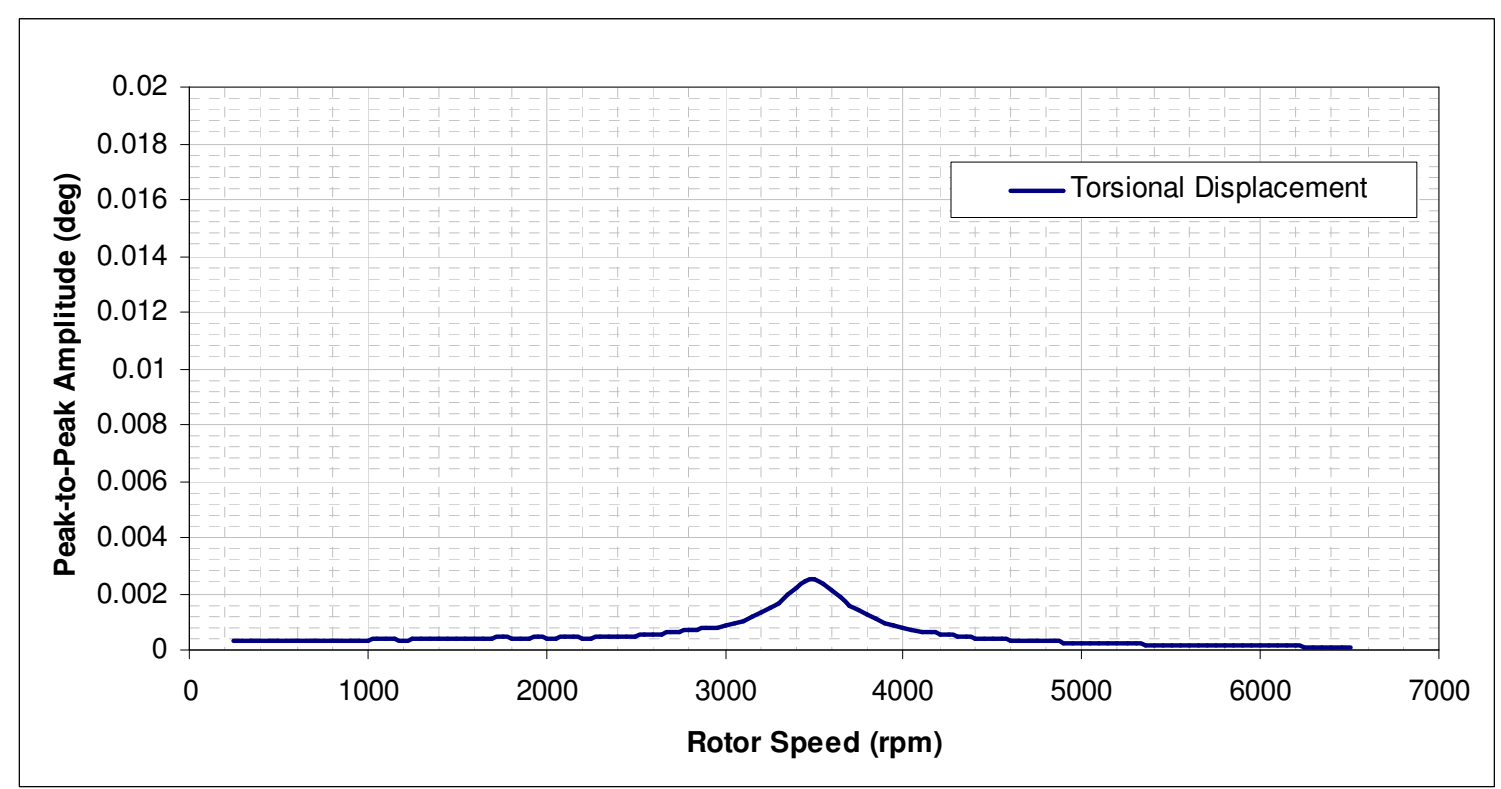

Figure 11: Torsional vibration amplitude vs. speed for gravitationally loaded rotor 


\section{III.3 General Vibration}

A general vibration model is required to consider torsional and lateral vibration interaction. To simplify simulation, rigid coupling ( $\theta=0)$, no horizontal side load $\left(P_{X}=0\right)$, and $\delta=0$ are assumed. For the purpose of numerical modeling, let the states $u_{1}=\varphi, u_{2}=\varphi^{\prime}, u_{3}=X_{M}, u_{4}=X_{M}^{\prime}, u_{5}=Y_{M}, u_{6}=Y_{M}^{\prime}$. The equations of motion Eq. II-16 become:

$$
\begin{aligned}
u_{1}^{\prime}=u_{2} \\
u_{2}^{\prime}=-\left(\frac{\omega_{t}}{\omega_{n}}\right)^{2} u_{1}-\frac{2 \varsigma_{t} \omega_{t}}{\omega_{n}} u_{2}-\frac{\varepsilon q}{\omega_{n}^{2} \rho^{2}}\left(\frac{P_{Y}}{M}\right) \cos \left(\frac{\Omega}{\omega_{n}} \tau+u_{1}\right)+\frac{q}{\omega_{n}^{4} \rho^{2}} \sin \left(2\left(\frac{\Omega}{\omega_{n}} \tau+u_{1}\right)\right)\left(\frac{P_{Y}}{M}\right)^{2} \\
+\frac{\varepsilon 2 \varsigma}{\rho^{2}}\left[\cos \left(\frac{\Omega}{\omega_{n}} \tau+u_{1}\right) u_{6}-\sin \left(\frac{\Omega}{\omega_{n}} \tau+u_{1}\right) u_{4}\right]+\frac{\varepsilon(1+q)}{\rho^{2}}\left[\cos \left(\frac{\Omega}{\omega_{n}} \tau+u_{1}\right) u_{5}-\sin \left(\frac{\Omega}{\omega_{n}} \tau+u_{1}\right) u_{3}\right] \\
-\frac{2 q}{\omega_{n}^{2} \rho^{2}}\left(\frac{P_{Y}}{M}\right)\left[\cos \left(2\left(\frac{\Omega}{\omega_{n}} \tau+u_{1}\right)\right) u_{3}+\sin \left(2\left(\frac{\Omega}{\omega_{n}} \tau+u_{1}\right)\right) u_{5}\right] \\
+\frac{q}{\rho^{2}}\left[2 \cos \left(2\left(\frac{\Omega}{\omega_{n}} \tau+u_{1}\right)\right) u_{3} u_{5}-\sin \left(2\left(\frac{\Omega}{\omega_{n}} \tau+u_{1}\right)\right) u_{3}^{2}+\sin \left(2\left(\frac{\Omega}{\omega_{n}} \tau+u_{1}\right)\right) u_{5}^{2}\right] \\
+u_{4}^{\prime}=-2 \varsigma u_{4}-\left(1-q \cos \left(2\left(\frac{\Omega}{\omega_{n}} \tau+u_{1}\right)\right)\right) u_{3}+q \sin \left(2\left(\frac{\Omega}{\omega_{n}} \tau+u_{1}\right)\right) u_{5}+\varepsilon u_{2}^{\prime} \sin \left(\frac{\Omega}{\omega_{n}} \tau+u_{1}\right) \\
+\frac{\varepsilon}{\omega_{n}^{2}}\left(\Omega+\omega_{n} u_{2}\right)^{2} \cos \left(\frac{\Omega}{\omega_{n}} \tau+u_{1}\right)-\left(\frac{P_{Y}}{M}\right) \frac{q}{\omega_{n}^{2}} \sin \left(2\left(\frac{\Omega}{\omega_{n}} \tau+u_{1}\right)\right) \\
u_{6}^{\prime}=-2 \varsigma u_{6}-\left(1+q \cos \left(2\left(\frac{\Omega}{\omega_{n}} \tau+u_{1}\right)\right)\right) u_{5}+q \sin \left(2\left(\frac{\Omega}{\omega_{n}} \tau+u_{1}\right)\right) u_{3}-\varepsilon u_{2}^{\prime} \cos \left(\frac{\Omega}{\omega_{n}} \tau+u_{1}\right) \\
u_{5}^{\prime}=u_{6} u_{2}^{2} \sin \left(\frac{\Omega}{\omega_{n}} \tau+u_{1}\right)+\left(\frac{P_{Y}}{M}\right) \frac{q}{\omega_{n}^{2}} \cos \left(2\left(\frac{\Omega}{\omega_{n}} \tau+u_{1}\right)\right)
\end{aligned}
$$

Eq. III-5

The Matlab programming found in Appendix C.4 is used to find a solution to Eq. III-5. The predicted response amplitudes for the parameters in Table 4 are plotted in Figure 12. 
Table 4: Parameters used for general vibration model

\begin{tabular}{|l|l|l|l|l|l|l|l|l|}
\hline$\omega_{n}$ & $\omega_{\mathrm{t}}$ & $\varsigma$ & $\varsigma_{\mathrm{t}}$ & $\varepsilon$ & $\rho$ & $\mathrm{Py} / \mathrm{M}$ & $\mathrm{q}$ & $\frac{P_{Y}}{k} / \varepsilon$ \\
\hline $1700 \mathrm{rpm}$ & $2500 \mathrm{rpm}$ & 0.2 & 0.04 & $0.001 \mathrm{in}$ & $1.0 \mathrm{in}$ & $1000 \mathrm{in} / \mathrm{sec}^{2}$ & 0.2 & 31.5 \\
\hline
\end{tabular}

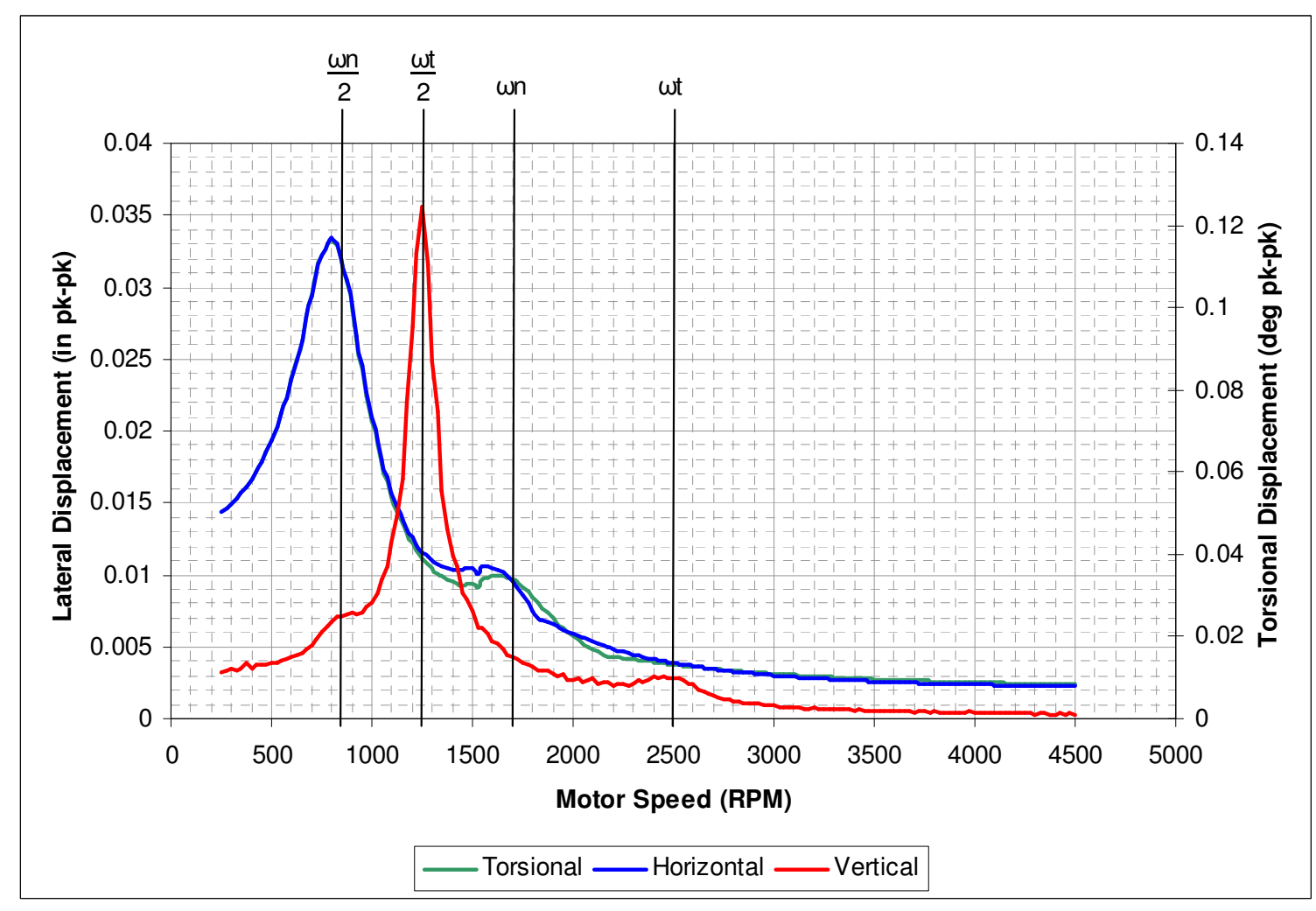

Figure 12: Response amplitudes predicted by general vibration model

Lateral response amplitude peaks around $1 / 2 \omega_{n}$ and $\omega_{n}$ as predicted by the simplified model in II.4.

Torsional response amplitude peaks at $1 / 2 \omega_{t}$ and $\omega_{t}$ as predicted by the simplified model in II.5. However new peaks appear in the torsional response at $1 / 2 \omega_{n}$ and $\omega_{n}$ that were not predicted by the simplified model. These peaks are associated with the interaction between the lateral and torsional vibration introduced by the shaft asymmetry.

The following chapters will attempt to identify trends in the torsional and lateral vibration. 


\section{III.4 Trends of Increasing Shaft Asymmetry}

The general vibration model developed in III.3 may be useful to understand how a developing shaft asymmetry affects the vibration of a rotor, for example in the case of a propagating crack. As defined in II.2, the asymmetry factor, $q$ represents the relative difference in stiffness between the strong and weak axes of the shaft. $q=0$ represents a perfectly symmetric shaft while increasing values of $q$ represent increasing asymmetry. If the stiffness in the strong direction is assumed constant $\left(k_{\eta}=\right.$ constant $)$, the asymmetry factor $q$ would increase as the stiffness in the weak direction $\left(k_{\xi}\right)$ decreases. This would reduce the average stiffness $(k)$ and therefore reduce the lateral natural frequency, $\omega_{n}$.

Based on these assumptions, the decrease in $\omega_{n}$ is related to the increase in $q$ as follows

$$
\omega_{n}=\sqrt{\frac{1}{1+q}} \omega_{n, \text { original }}
$$

Eq. III-6

where $\omega_{n, \text { original }}$ is the lateral natural frequency before introduction of asymmetry.

For the purpose of this study, it is assumed that the source of the lateral stiffness asymmetry does not affect the torsional stiffness and therefore $\omega_{t}=$ constant . The Matlab model show in Appendix C.5 is used to model a rotor with the parameters in Table 5 and increasing shaft asymmetry factor, $q$. The resulting torsional vibration amplitudes are plotted in Figure 13 and Figure 14.

Table 5: Parameters used in vibration model of rotor with increasing shaft asymmetry

\begin{tabular}{|l|l|l|l|l|l|l|}
\hline$\omega_{n, \text { original }}$ & $\omega_{t}$ & $\varsigma$ & $\varsigma_{t}$ & $\varepsilon$ & $\rho$ & $\mathrm{Py} / \mathrm{M}$ \\
\hline $1850 \mathrm{rpm}$ & $2500 \mathrm{rpm}$ & 0.2 & 0.04 & $0.005 \mathrm{in}$ & $1.0 \mathrm{in}$ & $386.1 \mathrm{in} / \mathrm{sec}^{2}$ \\
\hline
\end{tabular}




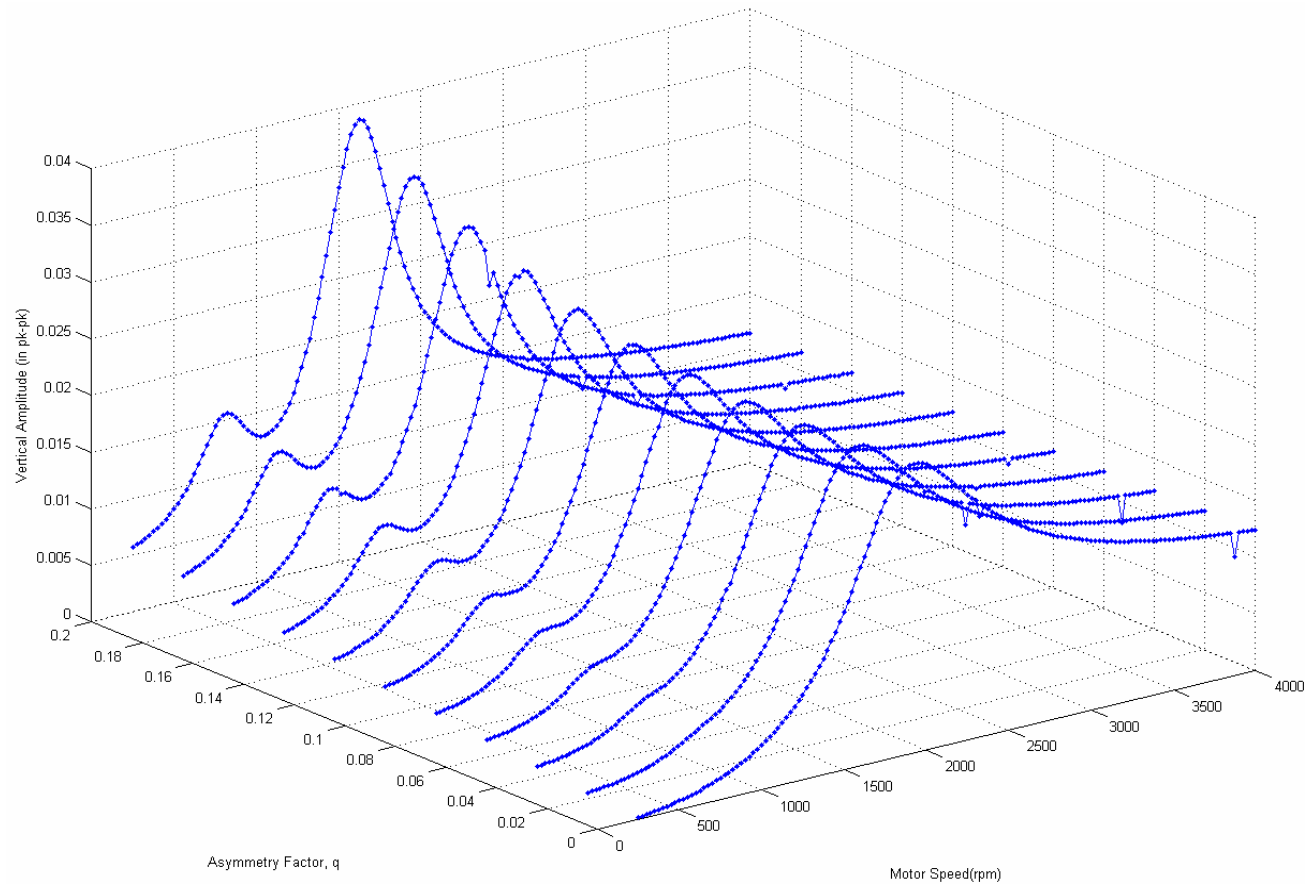

Figure 13: Vertical vibration amplitudes for rotor with increasing shaft asymmetry

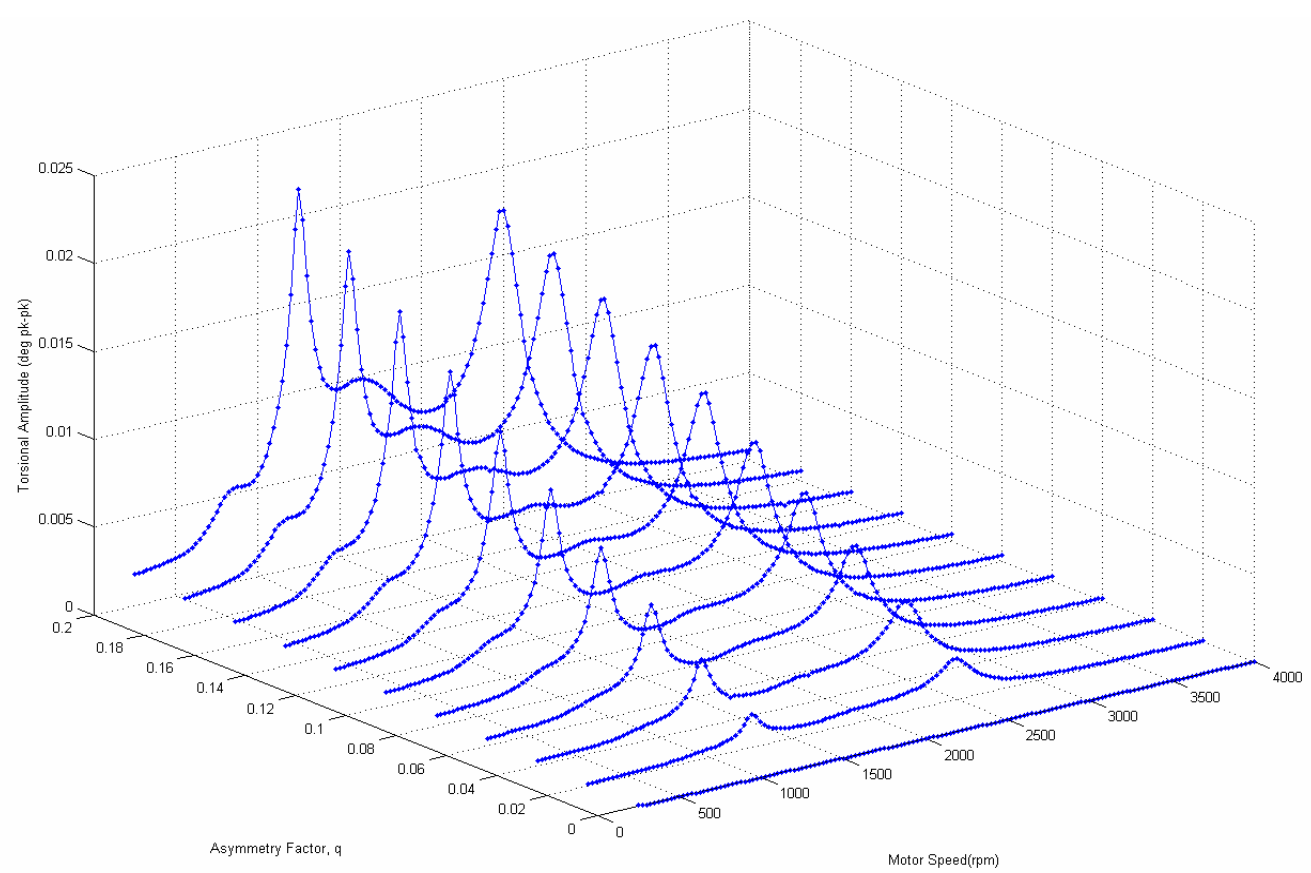

Figure 14: Torsional vibration amplitudes for rotor with increasing shaft asymmetry 
Figure 13 shows that lateral response at $1 / 2 \omega_{n}$ grows with increasing asymmetry, as does the response at $\omega_{n}$. However there is no apparent lateral response associated with torsional resonances at $1 / 2 \omega_{t}$ and $\omega_{t}$. As indicated by Eq. III-6, increase in $q$ reduces $\omega_{n}$ - an effect more apparent in the 2D plot.

It can be seen in Figure 14 that torsional response peaks at $1 / 2 \omega_{n}, \omega_{n}, 1 / 2 \omega_{t}$ and $\omega_{t}$ grow with increasing asymmetry. For low values of $q$, the peak lateral responses at $\omega_{n}$ and $1 / 2 \omega_{n}$ do not correlate with significant torsional response, despite proximity in motor speed to $\omega_{t}$ and $1 / 2 \omega_{t}$. However Torsional response at $\omega_{t}$ and $1 / 2 \omega_{t}$ associated with the unbalance and side load respectively are significant even at low values of $q$. Torsional response at $1 / 2 \omega_{t}$ appears to grow faster with increasing $q$ than the response at $\omega_{t}$. For higher values of $q$, lateral vibration at $1 / 2 \omega_{n}$ and $\omega_{n}$ is reflected in peaks in torsional response.

The orbit response at $1 / 2 \omega_{n}$ is plotted on the same scale for increasing values of $q$ in Figure 15 through Figure 19. Overall response grows with a characteristic two-loop pattern developing with increasing asymmetry. This is consistent with the predicted response of the simplified model presented in Figure 5

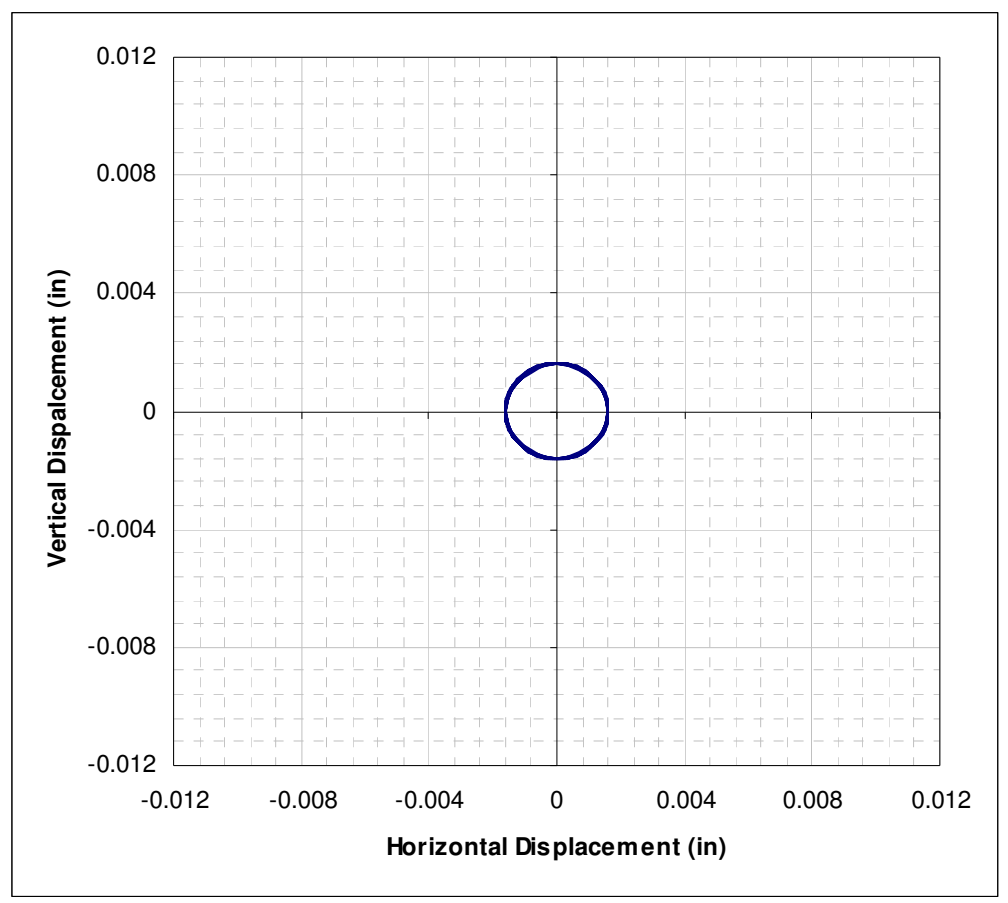

Figure 15: Orbit response at $1 / 2 \omega_{n}$ for $q=0$ 


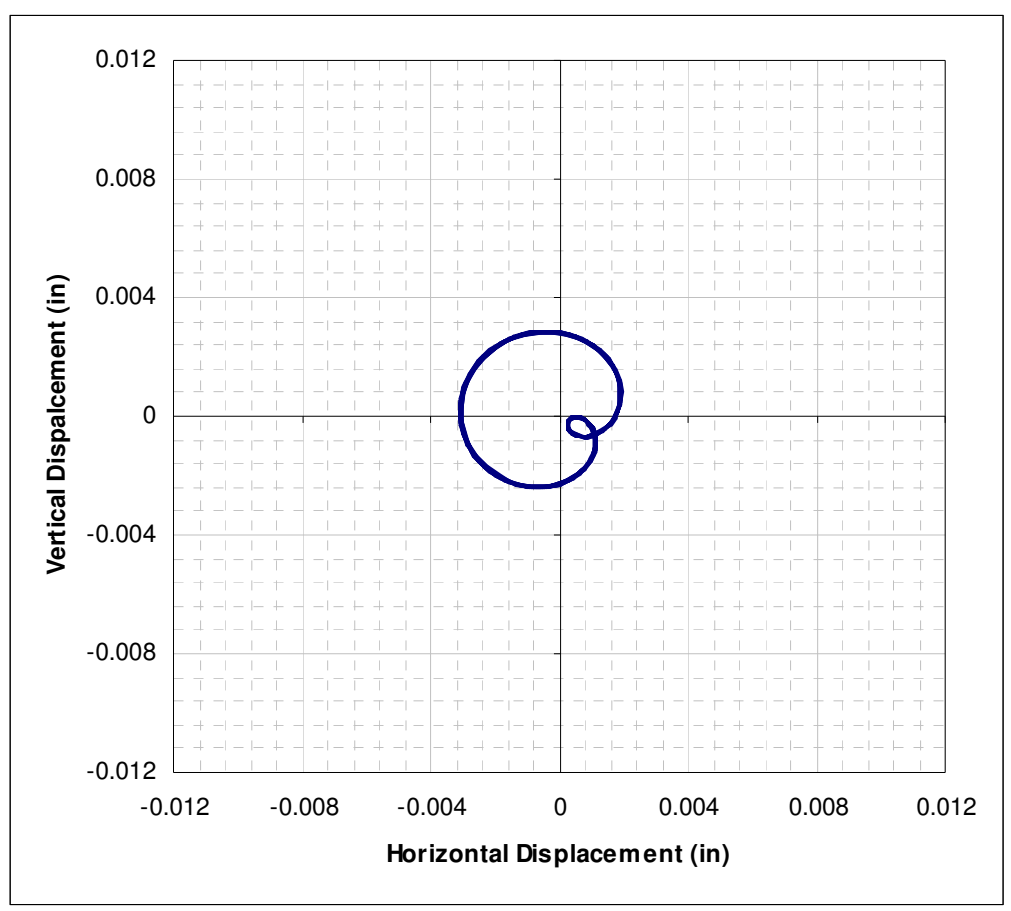

Figure 16: Orbit response at $1 / 2 \omega_{n}$ for $q=0.05$

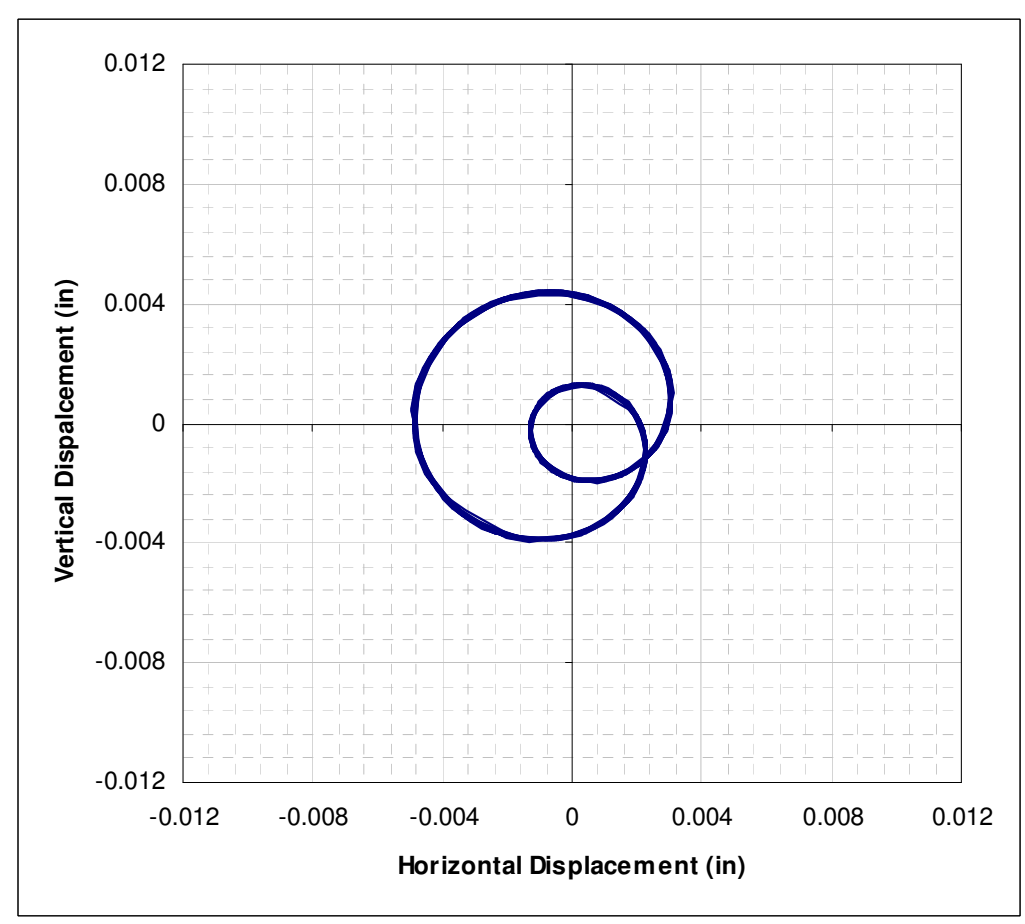

Figure 17: Orbit response at $1 / 2 \omega_{n}$ for $q=0.10$ 


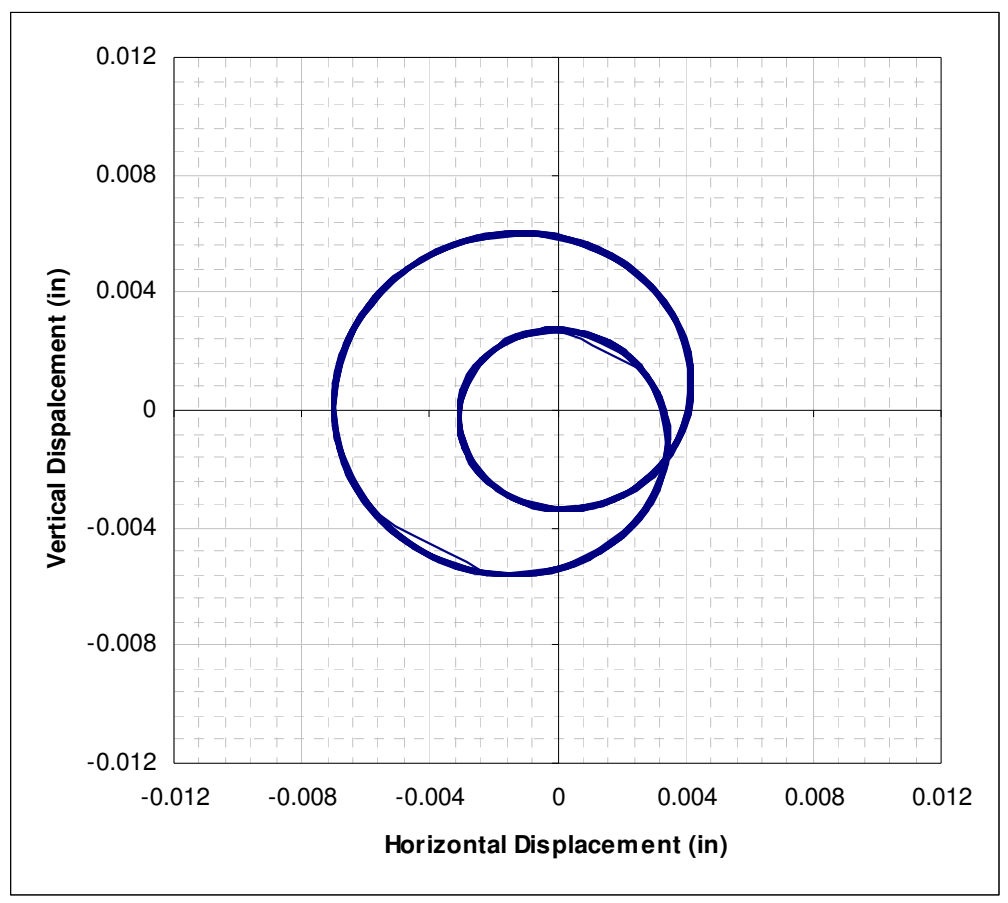

Figure 18: Orbit response at $1 / 2 \omega_{n}$ for $q=0.15$

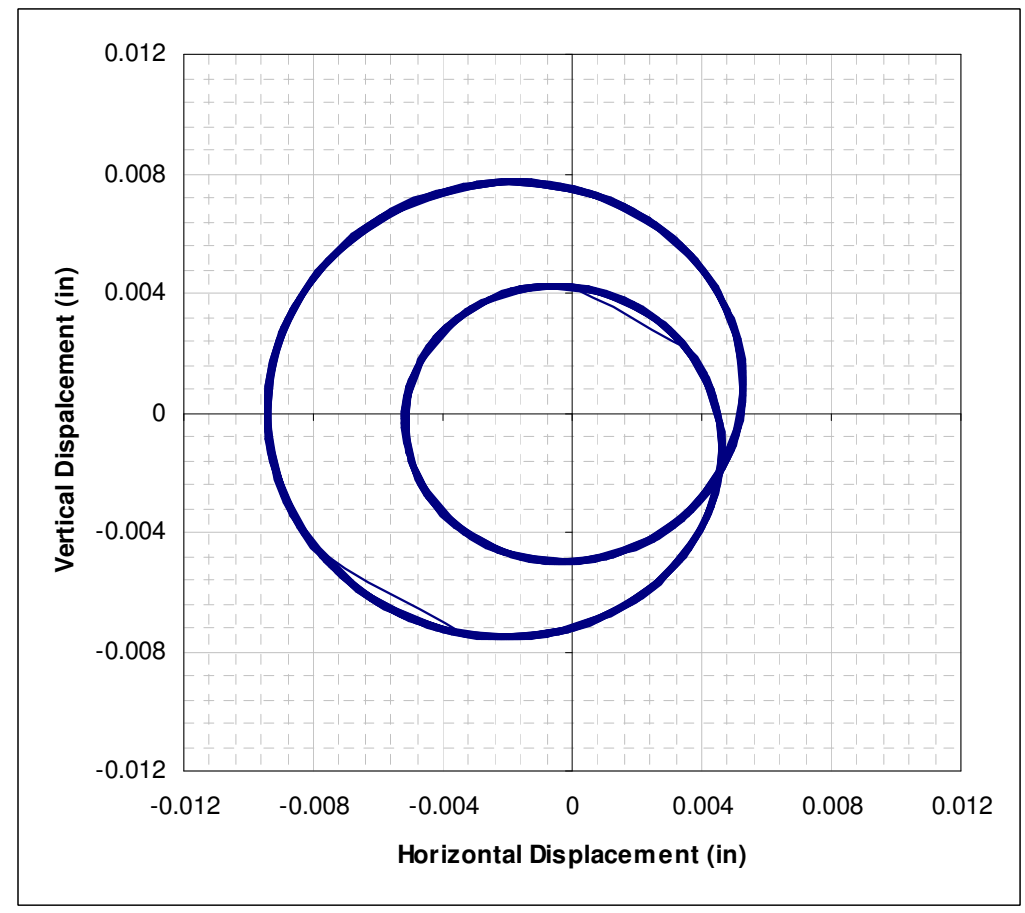

Figure 19: Orbit response at $1 / 2 \omega_{n}$ for $q=0.20$ 


\section{III.5 Effect on Response of the Proximity of $\omega_{t}$ to $\omega_{n}$}

To understand how torsional and lateral vibration interact, the model developed in III.3 is employed for varying ratios of $\omega_{t} / \omega_{n}$. Parameters from Table 5 are used, $\omega_{t}$ is varied and the asymmetry factor is held constant at $q=0.2$. Matlab programming found in Appendix C.6 is used to predict response, which is plotted in Figure 20, Figure 21 and Figure 22. Lateral response remains completely unchanged, as indicated by the overlapping plots in Figure 20. Torsional response at $\omega_{t}$ and $1 / 2 \omega_{t}$ both peak when $\omega_{t} / \omega_{n}=0.5$, when $\omega_{t}$ corresponds with $1 / 2 \omega_{n}$. Torsional response is less pronounced for $\omega_{t} / \omega_{n}=1$ and decreases as $\omega_{t} / \omega_{n}$ increases beyond $\omega_{t} / \omega_{n}=1$.

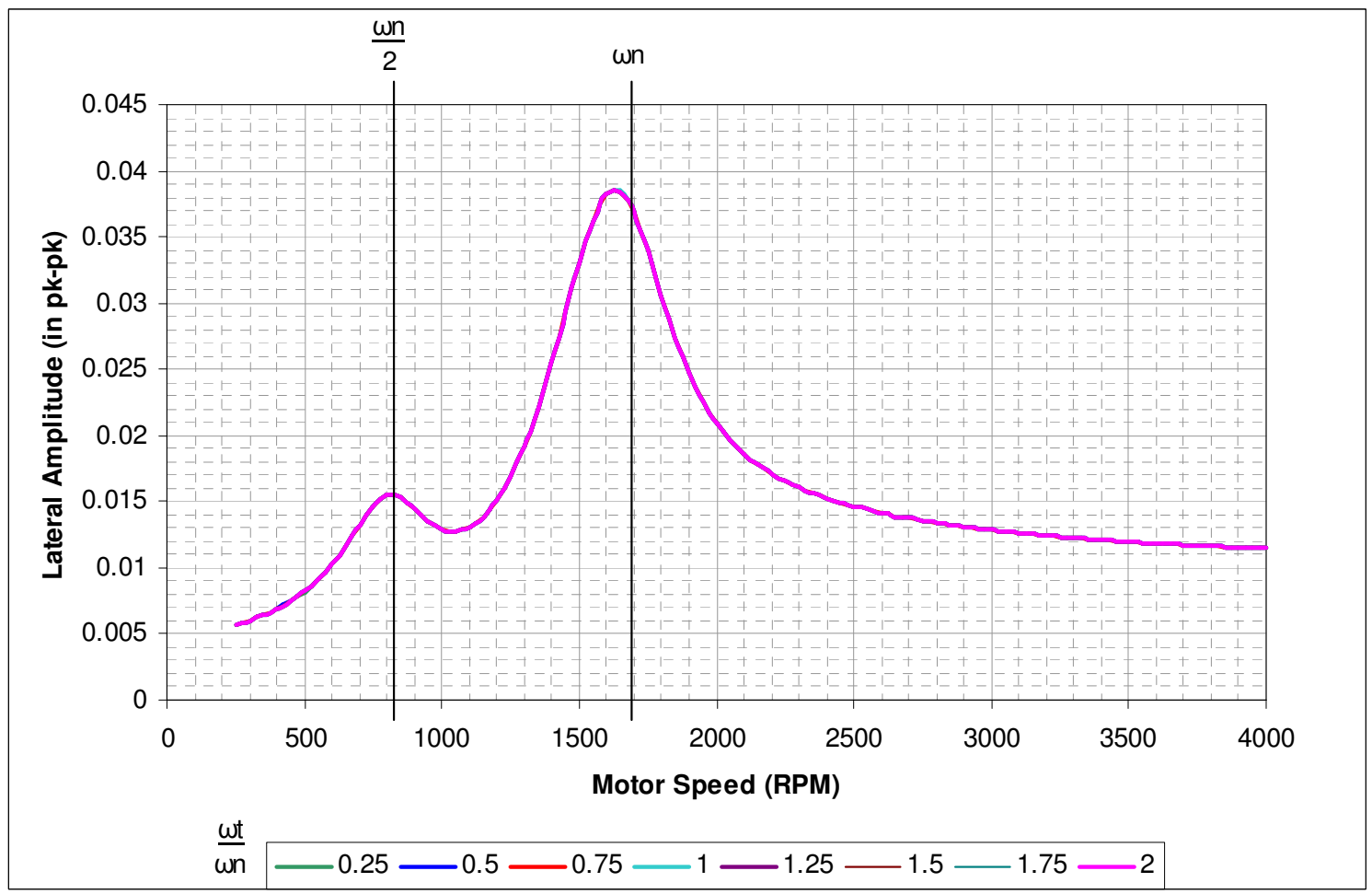

Figure 20: Vertical response for varying ratios of $\omega_{t} / \omega_{n}$ 


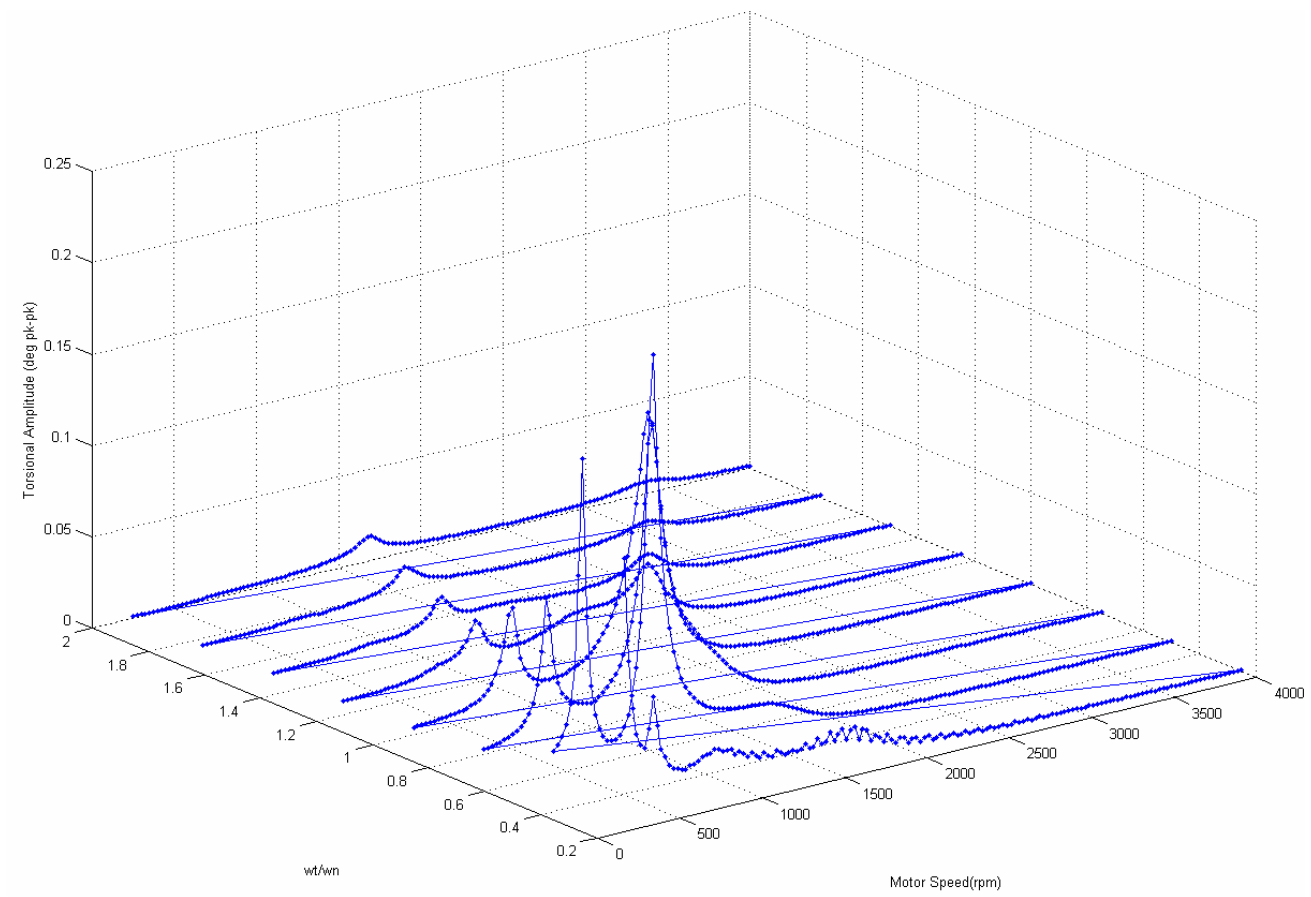

Figure 21: Torsional response for varying ratios of $\omega_{t} / \omega_{n}$ (3D plot)

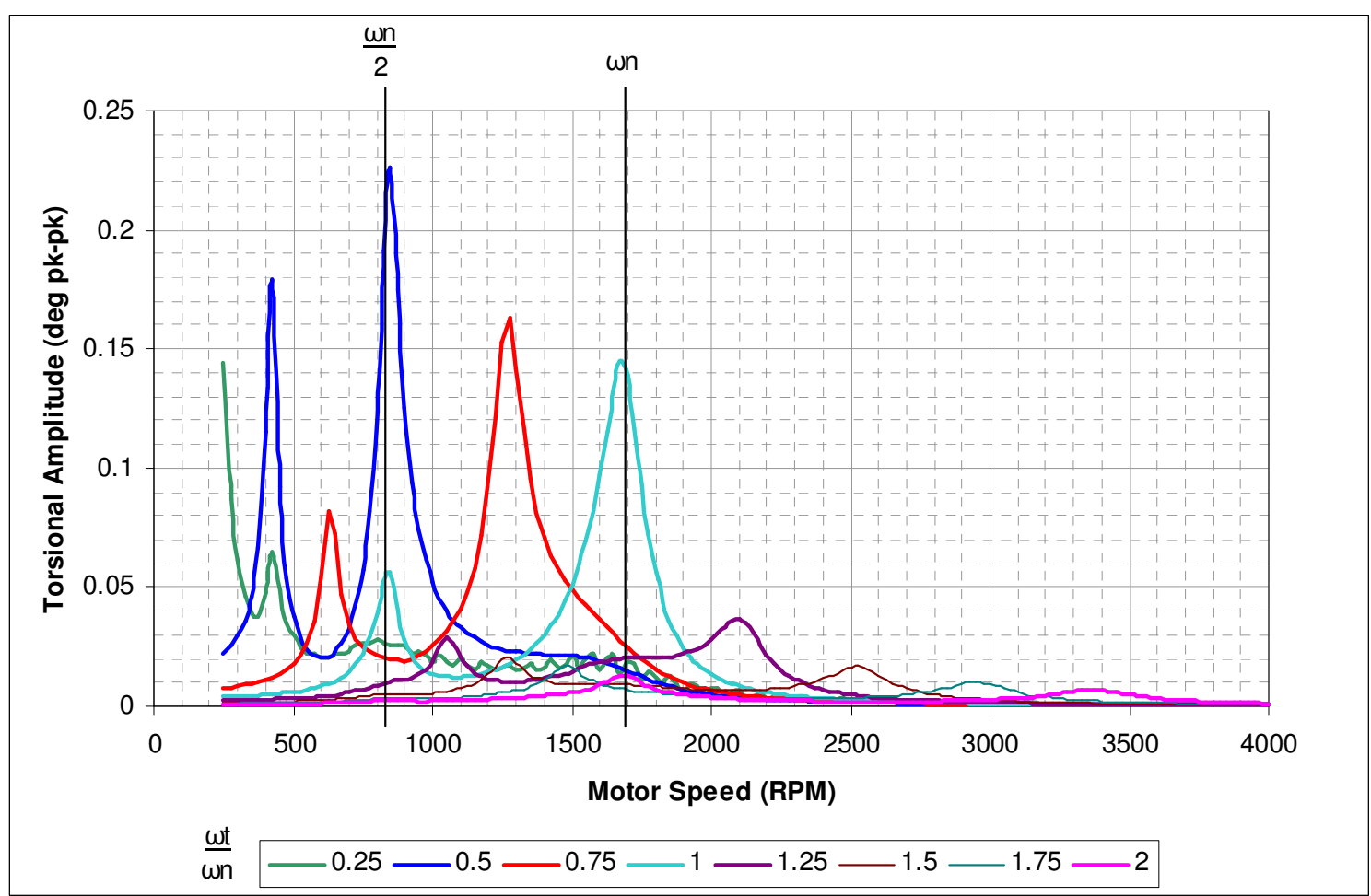

Figure 22: Torsional response for varying ratios of $\omega_{t} / \omega_{n}$ (2D plot) 


\section{III.6 Effect of Increasing Side Load}

The general vibration model developed in III.3 may be used to understand how a side load affects the vibration of an asymmetric shaft rotor. In real systems, side load may vary with aerodynamic or gearbox loads. Determining how changing loads are reflected in rotor response could help with diagnosis of an asymmetry. The Matlab model in Appendix C.7 is used to predict rotor response for varying loads and the response amplitudes are plotted in Figure 23 and Figure 24.

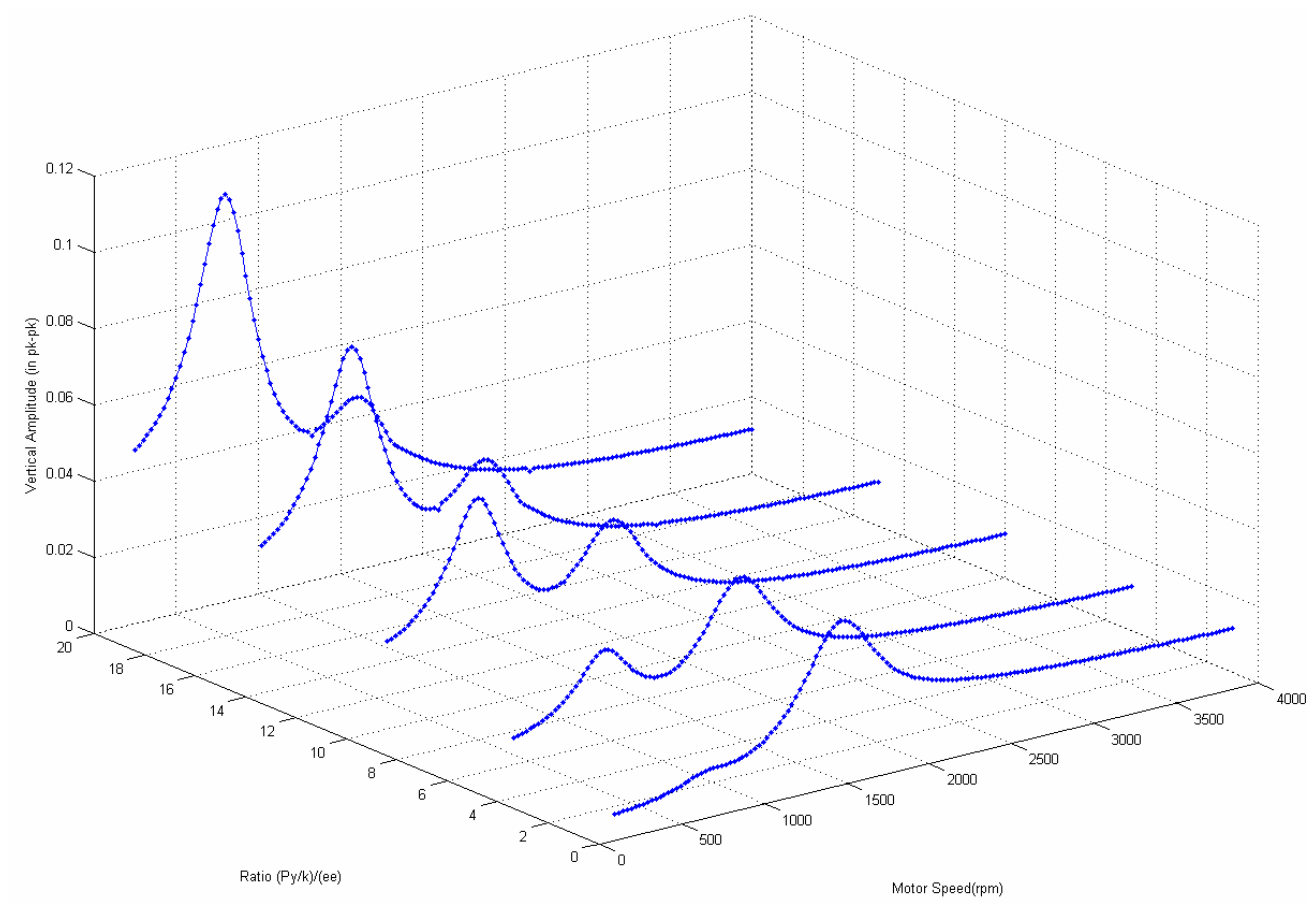

Figure 23: Lateral response for increasing side load (3D plot)

Lateral response is primarily affected at $1 / 2 \omega_{n}$ where overall response amplitude increases linearly with side load. Torsional response increases with side load at $1 / 2 \omega_{n}, 1 / 2 \omega_{t}$ and $\omega_{t}$, with the most notable sensitivity at $1 / 2 \omega_{t}$. Torsional resonances are not reflected in the lateral response. 


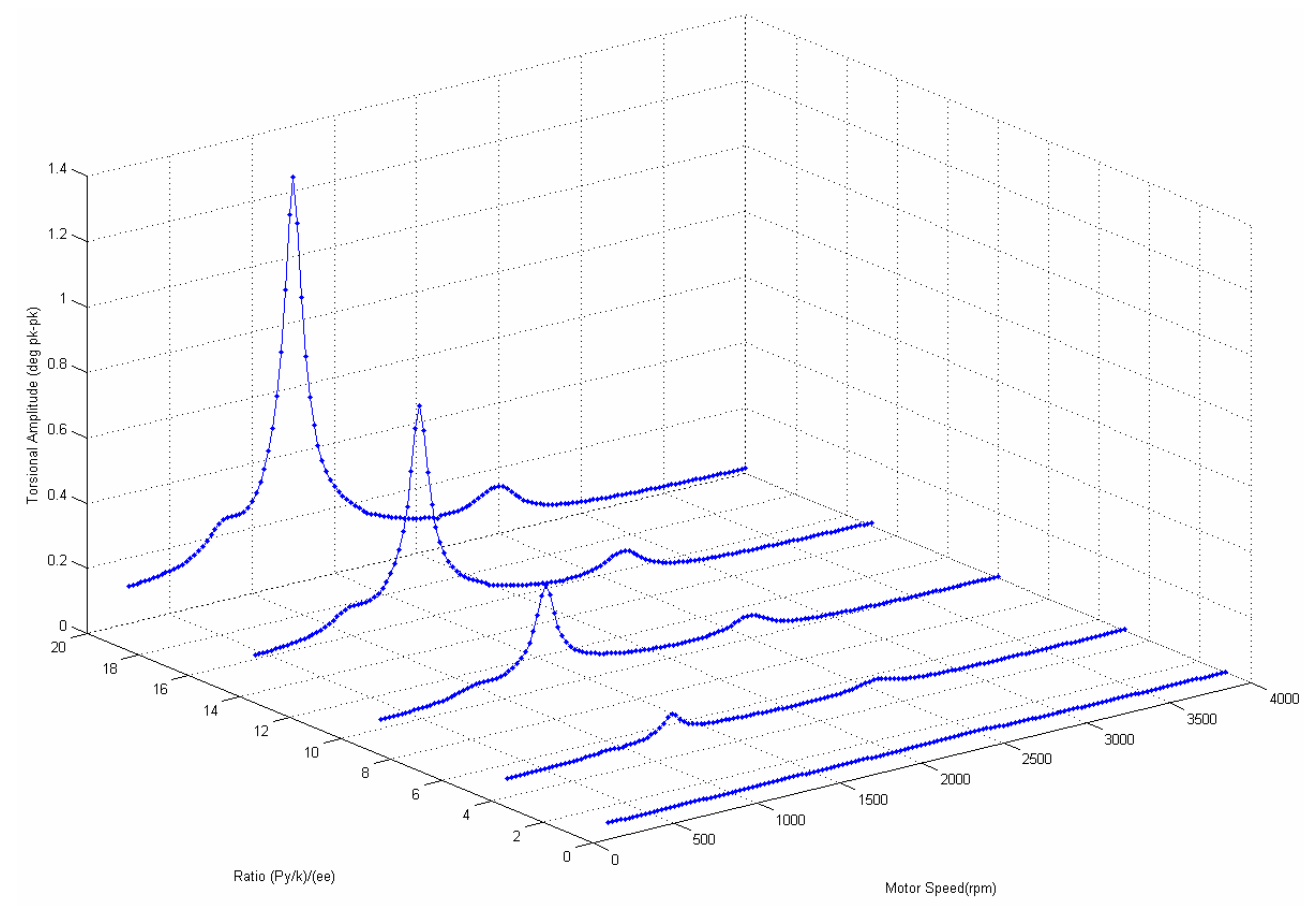

Figure 24: Torsional response for increasing side load (3D plot) 


\section{Chapter IV Experiment}

\section{IV.1 Overview of Experiment}

The objectives of the experiment are to provide information for verifying and improving the mathematical model, and to investigate the practical implications of lateral and torsional vibration measurement as diagnostic tools. To this aim, the experimental foundation is the Bently Nevada RK-4 Rotor Kit shown in Figure 25. This assembly allows for a wide range of configurations and is adaptable for the specific needs of this study. Two disks fixed to a common shaft and supported with plain rigid bearings are driven by an electric motor through a flexible coupling. A shaft-mounted bearing allows the application of a side load. The inboard disk is constrained by adjacent bearings to prevent lateral vibration. The outboard disk is supported by an adjustable bearing span to allow lateral flexibility. Torsional flexibility arises from the section of shaft connecting the two disks. Proximity probes are used to measure vibration and allow feedback control of rotor speed.

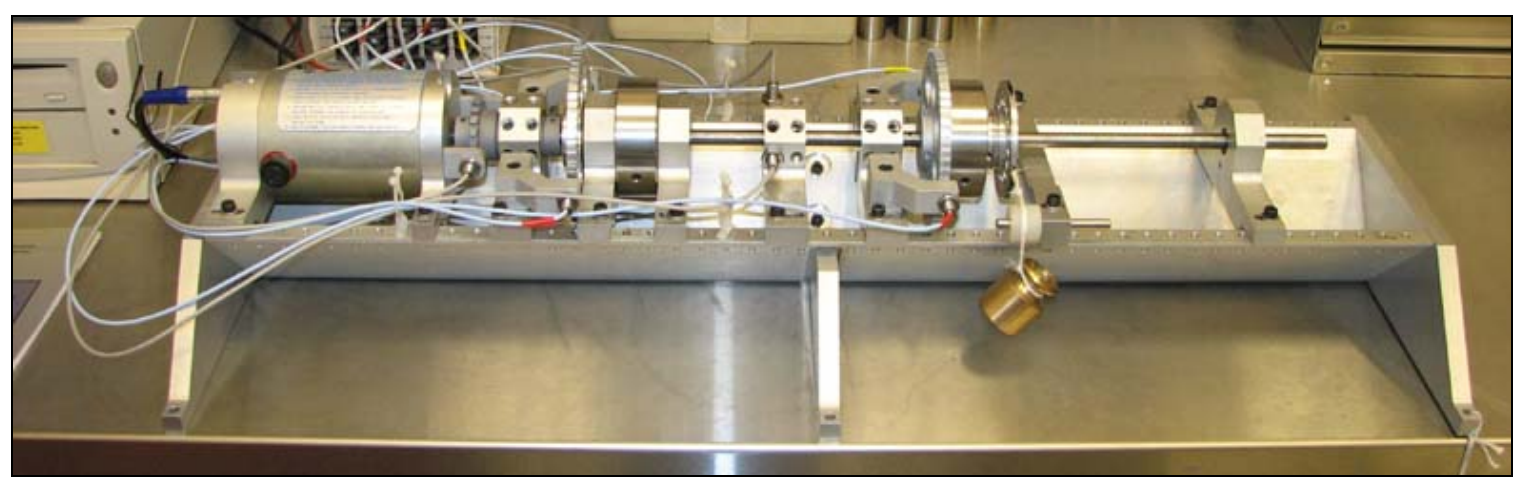

Figure 25: Bently Nevada RK-4 Rotor Kit configured for a side loaded rotor

Based on estimates from the sizes of disks and shafts used by the RK-4 rotor kit, the bearing span of the outboard mass can be configured to provide a lateral natural frequency as low as 2,500rpm or a torsional natural frequency as low as 5,700rpm. Additional disks can be added to further reduce both natural frequencies. Figure 25 shows the rotor configured for minimum lateral natural frequency while Figure 26 shows a configuration for minimizing the torsional natural frequency. 


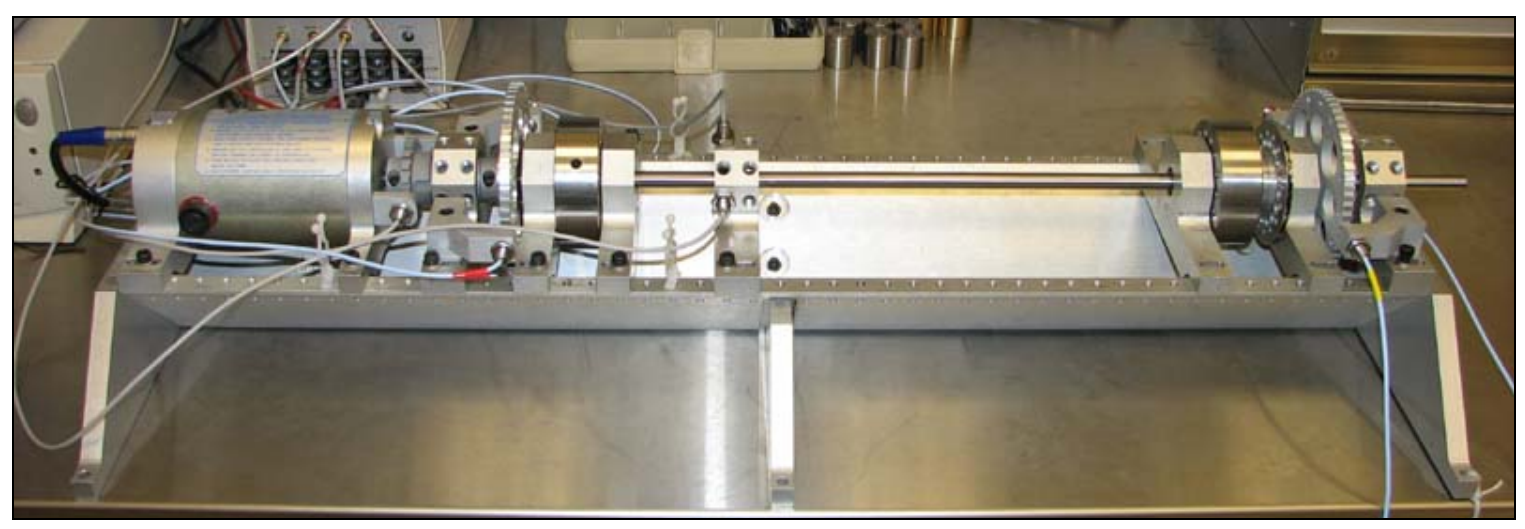

Figure 26: Rotor kit configured to minimize torsional natural frequency

The pulley system shown in Figure 27 is employed to provide an adjustable side load to the outboard rotor. Calibrated masses are hung from a string which draws over a pulley and loads a shaft-mounted bearing near the disk. Additional weights can be added to change loading conditions. Multiple pulleys can be used to load the shaft either the horizontal or vertical (gravitational) direction. The advantage of this system is flexibility to run a variety of side loads. However the masses hung have a potentially significant inertial influence on the rotor that is not modeled by this study. Use of a spring or magnetic load may be more appropriate to prevent inertial influence.

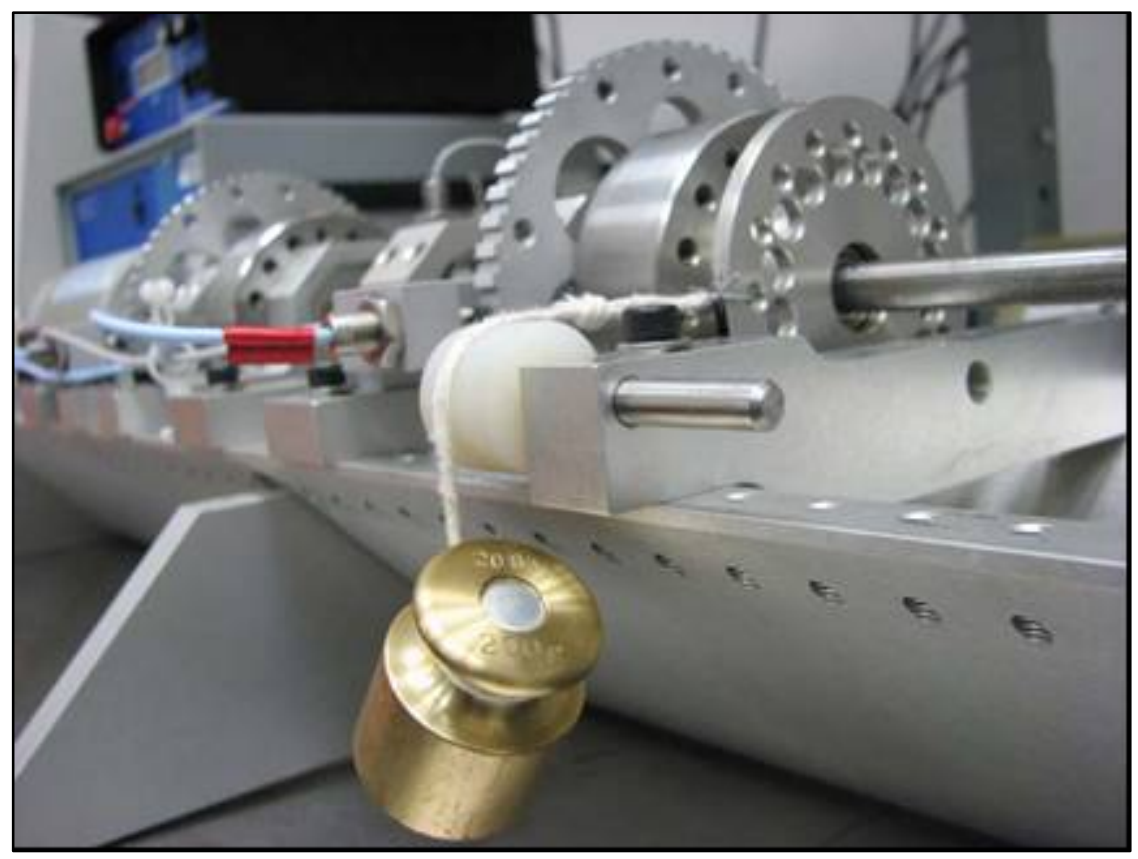

Figure 27: Side load provided by gravity through a pulley system 
To generate asymmetric lateral stiffness, four flats are carefully ground into the rotor shaft using a precision machine tool. These flats are oriented 180 degrees apart in two locations along the shaft. This provides symmetry about the center of the rotor's bearing span as well as axis-symmetric lateral stiffness. Varying flat depths result in varied magnitudes of stiffness asymmetry, as measured by load-deflection testing.

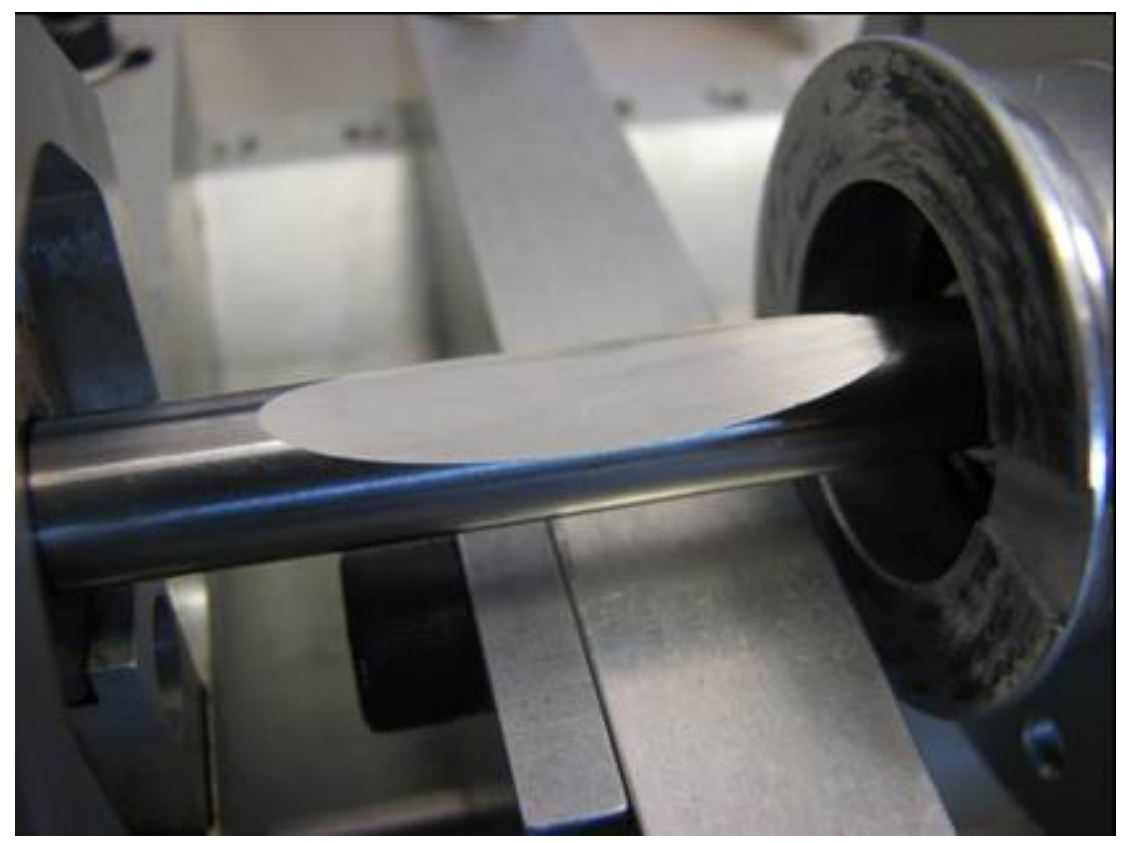

Figure 28: Precision ground flat in shaft to produce asymmetric stiffness

A direct current motor provides rotational power to the inboard end of the rotor system. A Bently Nevada RK4 Speed Controller uses feedback from a coupling-mounted reluctor wheel (shown in Figure 29) to control average motor rotational speed. Steady and ramped motor speeds can be achieved with this system. 


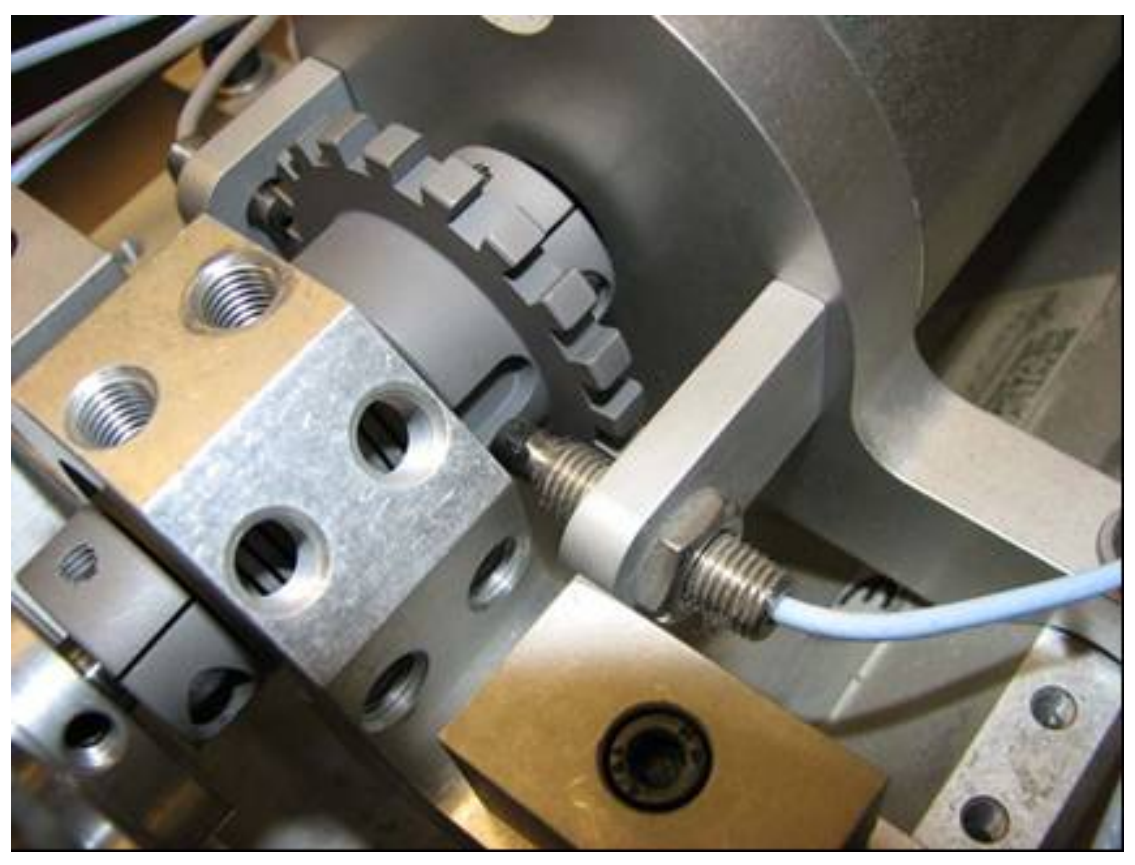

Figure 29: Reluctor wheel for motor speed control

Lateral vibration is measured directly at the shaft using eddy current proximity probes as shown in Figure 30. The probes are oriented mutually perpendicular to measure vertical and horizontal displacement respectively. Due to physical constraints and limitations in linear range, these probes may not always be positioned near a rotor. Instead they are placed along sections of shaft exhibiting acceptable levels of vibration. This vibration is assumed to be representative of the nearby rotor's vibration. Measurements made by these probes are assumed to be proportional to what would be measured at the rotor itself.

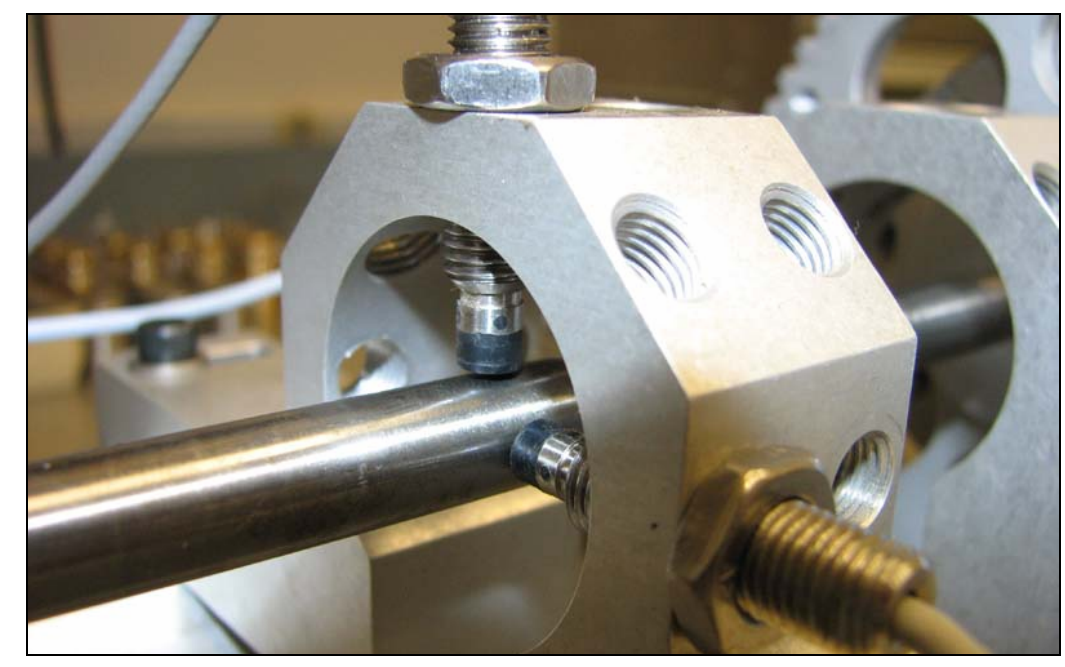

Figure 30: Proximity probes for lateral vibration measurement 
In addition to lateral probes, each disk is equipped with a transducer for measuring torsional vibration. The primary components are a custom machined gear wheel attached to the shaft and two proximity probes that are fixed 180 degrees apart in the horizontal plane, as shown in Figure 31.

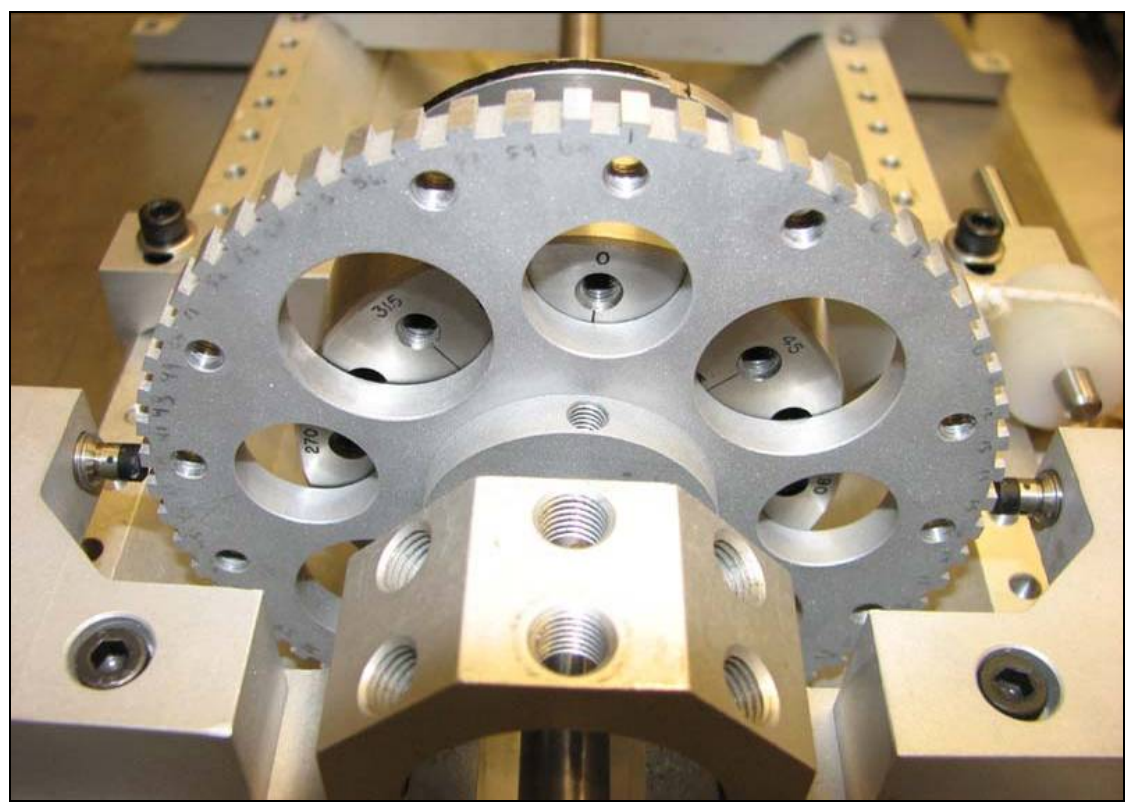

Figure 31: Torsional transducer consisting of a gear wheel and two proximity probes

The principle of operation of the torsional transducer can be described as follows. As the gear wheel rotates, proximity probes detect the passage of teeth. The time it takes for teeth to pass can be attributed to either lateral or torsional motion as follows:

$$
\Delta t_{L}=\frac{2 \pi R}{N\left(R \Omega_{i}+V_{y}\right)} \text { and } \Delta t_{R}=\frac{2 \pi R}{N\left(R \Omega_{i}-V_{y}\right)}
$$

where $\Omega_{i}$ is the instantaneous rotative speed, $V_{y}$ is the vertical velocity, $\mathrm{R}$ is the radius of the gear wheel, $\mathrm{N}$ is the number of teeth, and $\Delta t_{L}$ and $\Delta t_{R}$ are the times between pulses generated by the left and right probes respectively. The lateral component of the vibration signal $\left(V_{y}\right)$ can be eliminated from the above equations and the instantaneous rotative speed can be expressed as

$$
\Omega_{i}=\frac{\pi}{N}\left(\frac{\Delta t_{L}+\Delta t_{R}}{\Delta t_{L} \Delta t_{R}}\right)
$$


In general, the vibration of the disk can be express as

$$
\theta=\left(\Omega_{\text {motor }}-\Omega_{i}\right) t
$$

Eq. $I V-3$

where $\theta$ is the general vibration angle with respect to the motor and $\Omega_{\text {motor }}$ is the rotative speed of the motor.

\section{IV.2 Control, Signal Processing and Data Acquisition}

Bently Nevada equipment is widely used in industry for vibration monitoring and diagnostics. For this project, the RK-4 Rotor Kit is equipped with a motor speed controller to provide steady rotation at the inboard end of the rotor.

Two Bently Nevada TK17 Torsional Vibration Signal Conditions are employed to simplify measurement of torsional vibration and reduce post processing. Based on the relationships described in Eq. IV-1, Eq. IV-2 and Eq. IV-3, each signal conditioner processes the two proximity probe outputs of a torsional transducer into a single output proportional to torsional vibration amplitude.

For data acquisition, a Bently Nevada ADRE 208-P works in unison with a personal computer to store and process vibration signals in real time, allowing for a wide range of vibration monitoring and data storage. The 208-P performs many of the same functions of oscilloscopes, spectrum analyzers, filters, and recording instruments.

Seven signals are fed to the 208-P. Three signals come from both the inboard rotor and outboard rotor respectively. These include feedback from a vertical proximity probe, horizontal proximity probe, and torsional transducer (pre-processed by a TK17). A seventh signal from the rotor kit's Keyphasor provides a measurement of motor speed and vibration phase angle. 


\section{IV.3 Experimental Results}

In an attempt to excite torsional activity and lateral-torsional vibration interaction, the rotor kit is

configured as shown in Figure 32 (additional notes regarding this trial can be found in Appendix D.1). Two disks are used at both the inboard and outboard positions to reduce $\omega_{t}$ and $\omega_{n}$. The calculated values assuming rigid bearings and the dimensions of the rotor kit are $\omega_{t}=4,100 \mathrm{rpm}$ and $\omega_{n}=5,800 \mathrm{rpm}$. A very heavy side load is applied to achieve $\frac{P_{y}}{M}=2,600 \mathrm{in} / \mathrm{sec}^{2}$.

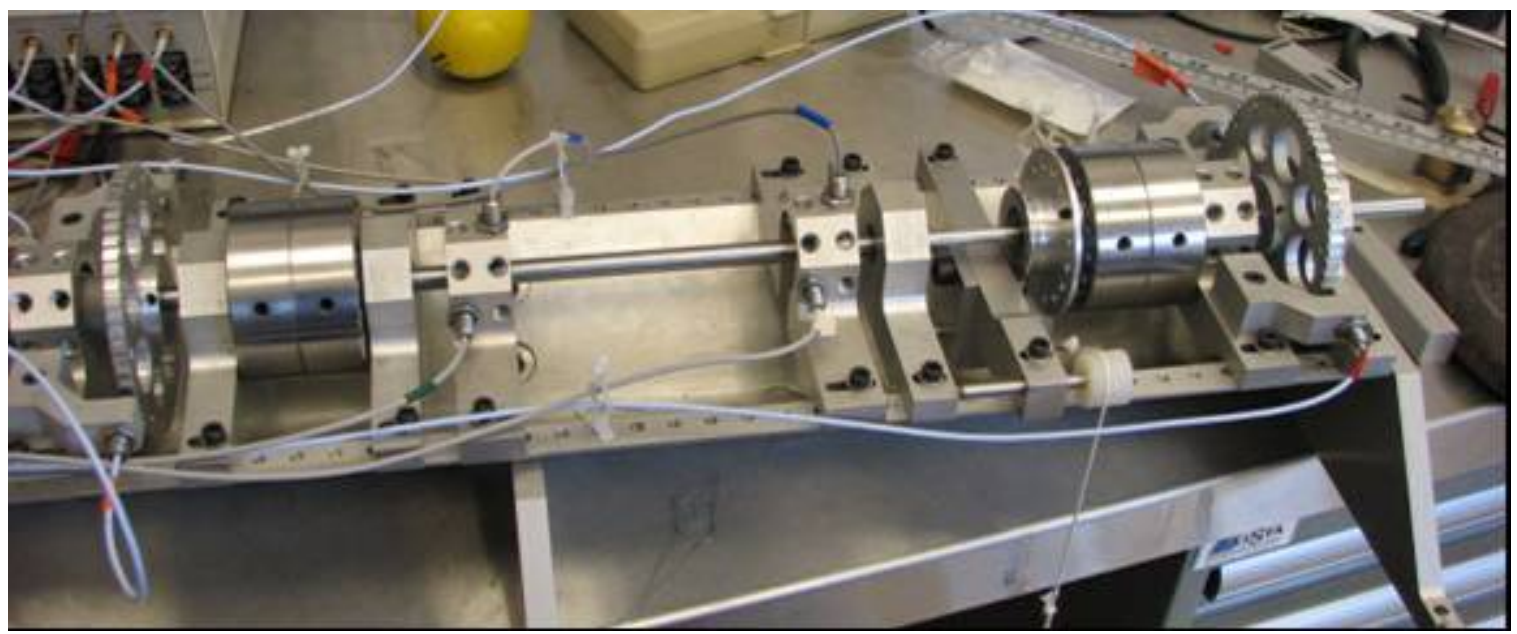

Figure 32: Rotor configuration for experimental results

The resulting vibration amplitudes as recorded by the ADRE 208-P are shown in the following figures. The direct and 1X lateral response of the outboard rotor is shown in Figure 33 and Figure 34. A broad 1X response peak is noted in the horizontal direction near 4,200rpm, but resonant peaks are not obvious in the vertical response. 


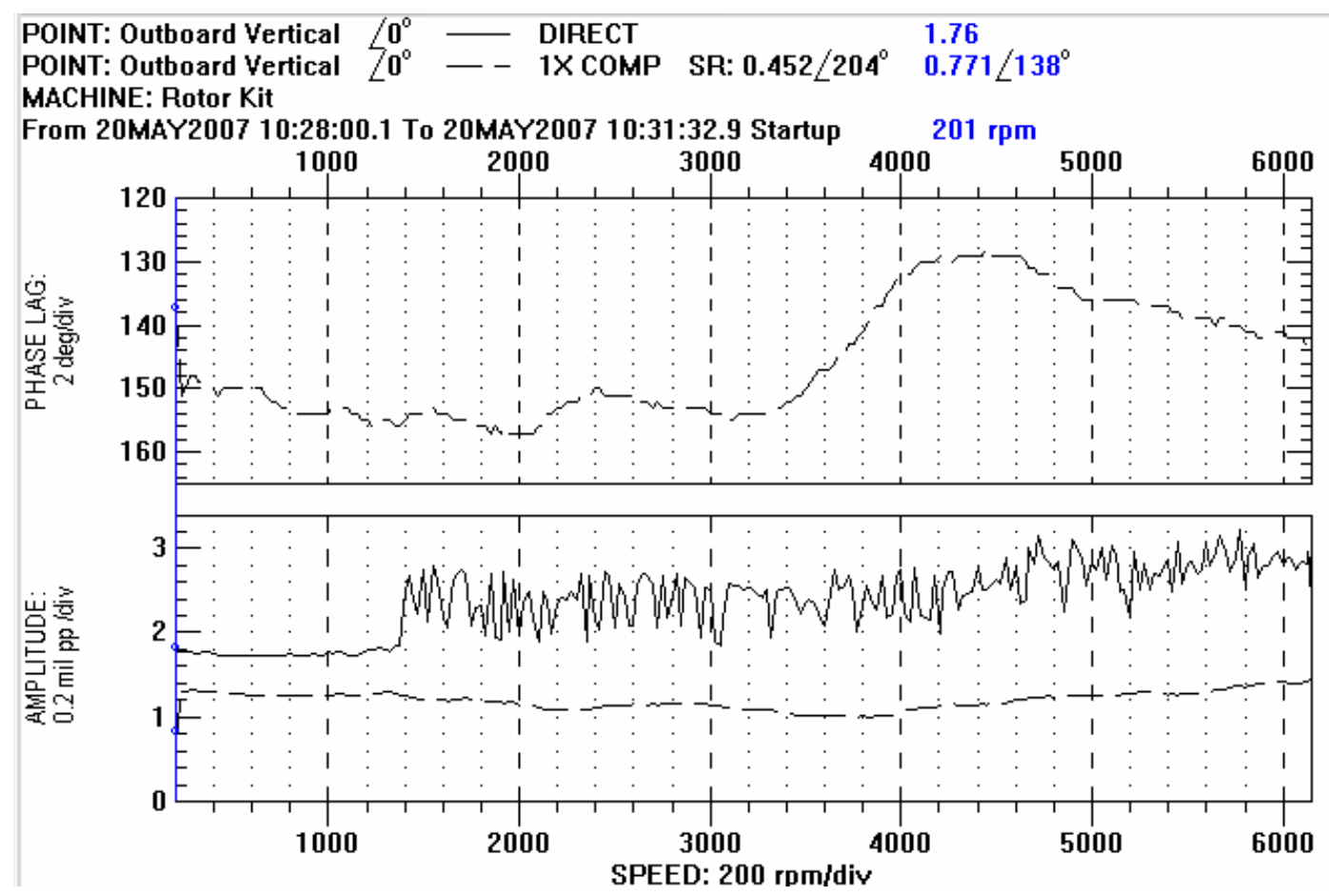

Figure 33: Vertical response, direct \& 1X

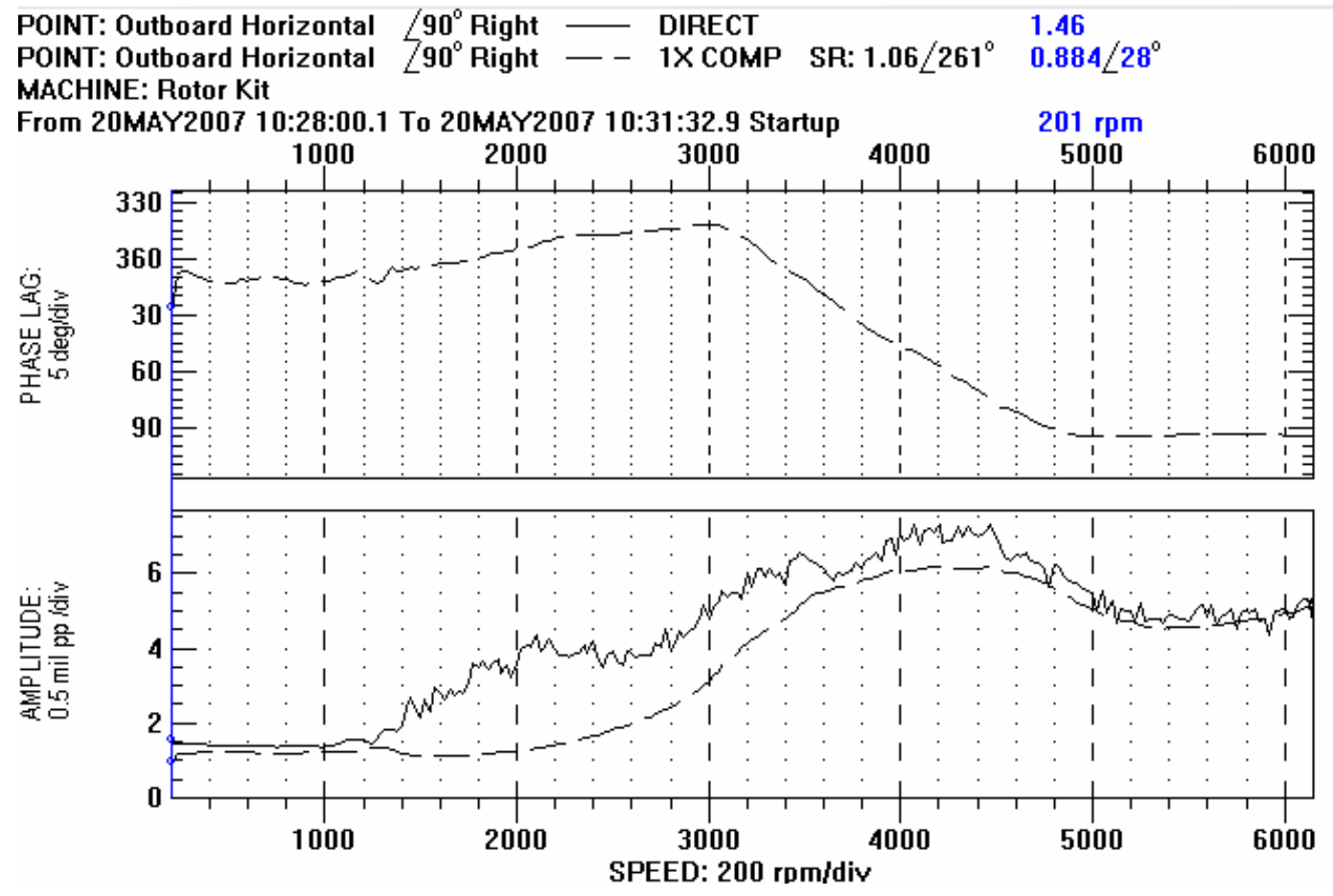

Figure 34: Horizontal response, direct \& 1X 
The $2 \mathrm{X}$ filtered lateral response shown in Figure 35 and Figure 36 shows two or more distinctive peaks, potentially related to the $1 \mathrm{X}$ and $2 \mathrm{X}$ excitations associated with the unbalance and side-load asymmetry interaction respectively. $2 \mathrm{X}$ response peaks appear to occur at different speeds for the horizontal and vertical directions. This may be an effect of the mass used for side-loading. It should be noted that this difference in response was not predicted in simulation.

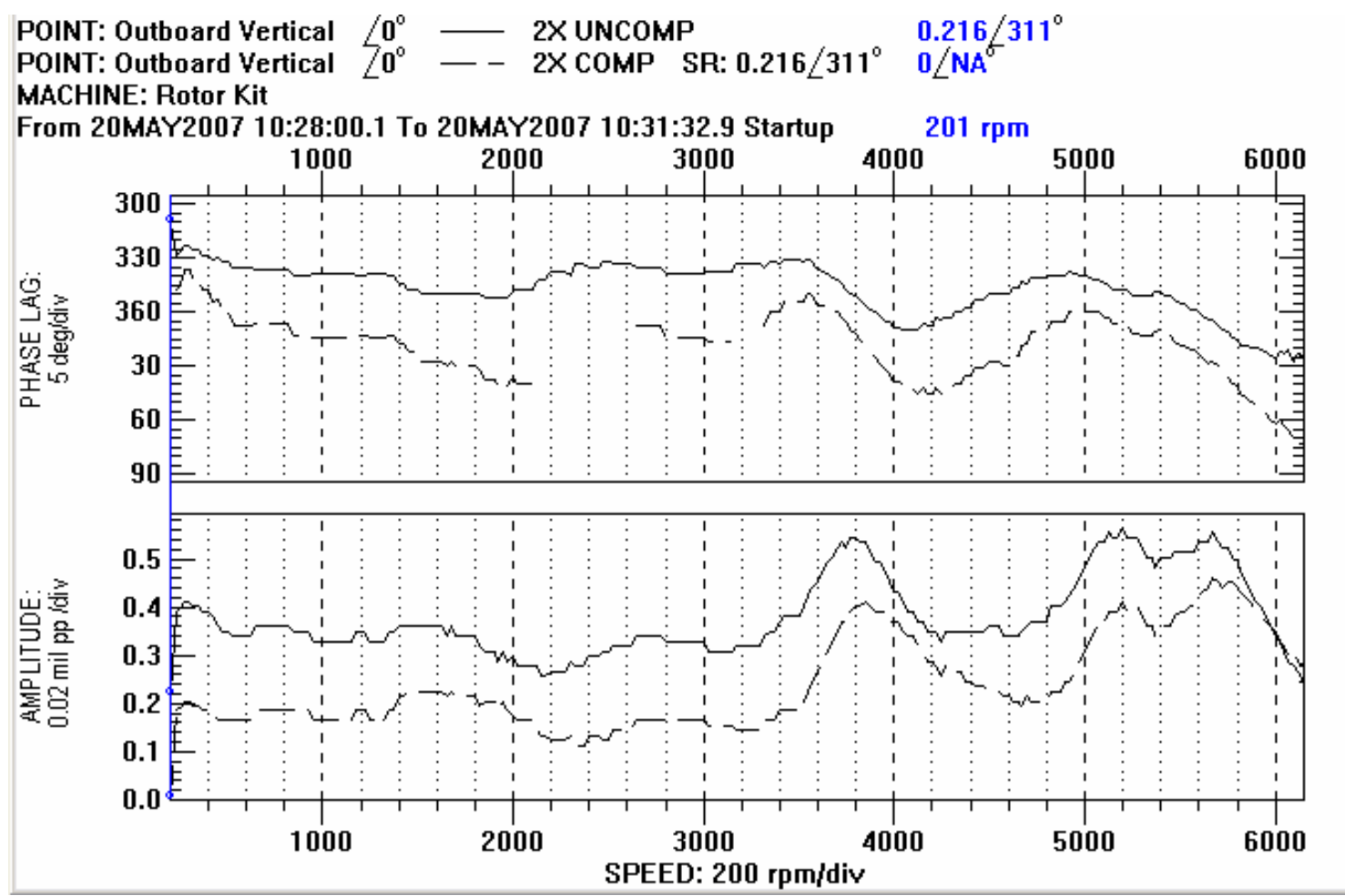

Figure 35: Vertical response, 2X only 


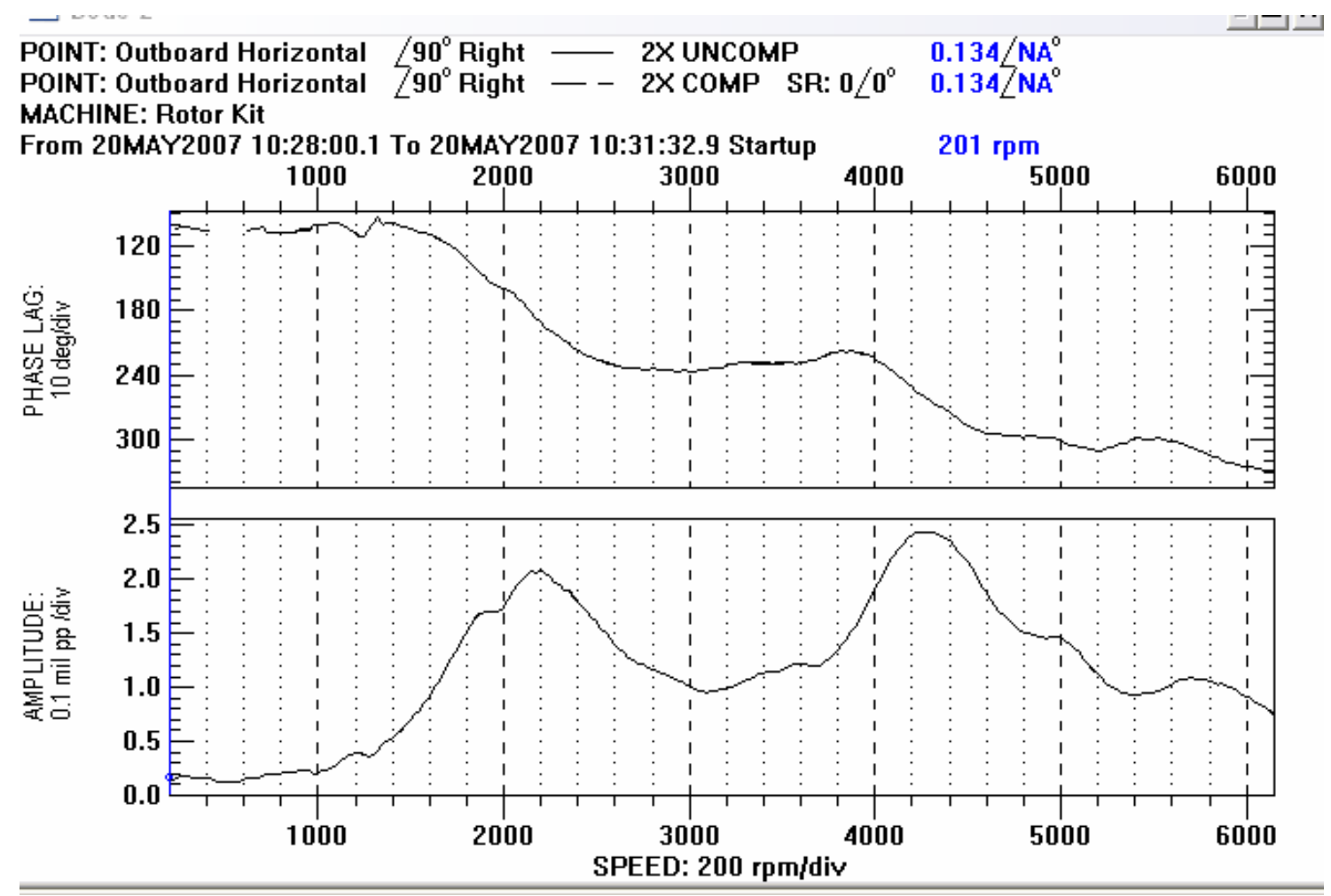

Figure 36: Horizontal response, $2 \mathrm{X}$ only

Torsional responses for the inboard and outboard disks separately are shown in Figure 37 and Figure 38.

The difference in the outboard and inboard response is shown in Figure 39. A 1X resonant peak is clearly shown at 5,400rpm, but no peak is obvious at half this speed, as might be expected from the simulation considering the high ratio of $\frac{P_{y}}{M}$. Observing the outboard rotor, some broad $1 \mathrm{X}$ response peaks also appear at about 900rpm and 3,600rpm, but these do not appear to correlate to either the natural frequency at 5,400 rpm or the peaks in lateral response. 


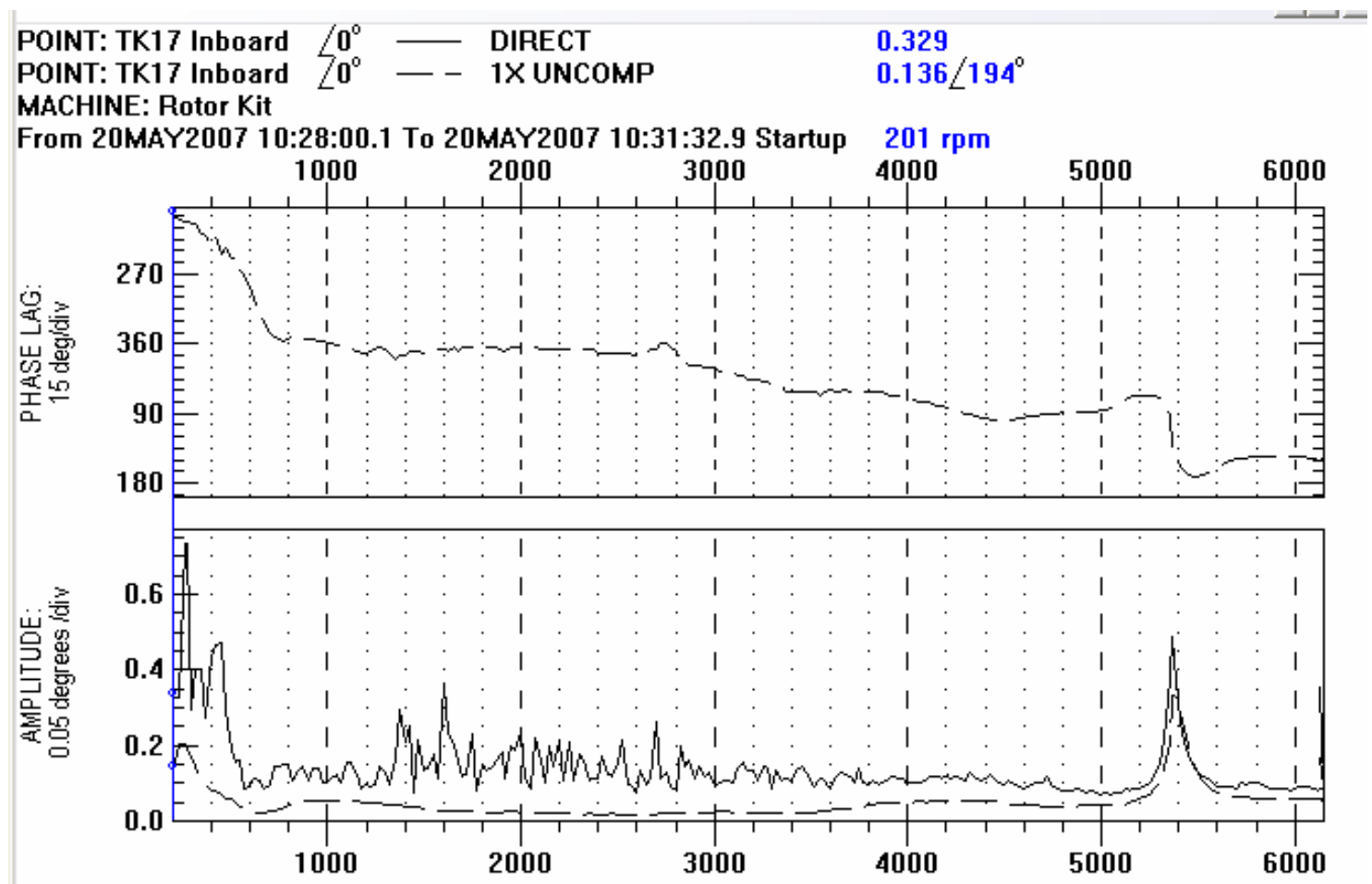

Figure 37: Torsional response of inboard disk, direct \& 1X

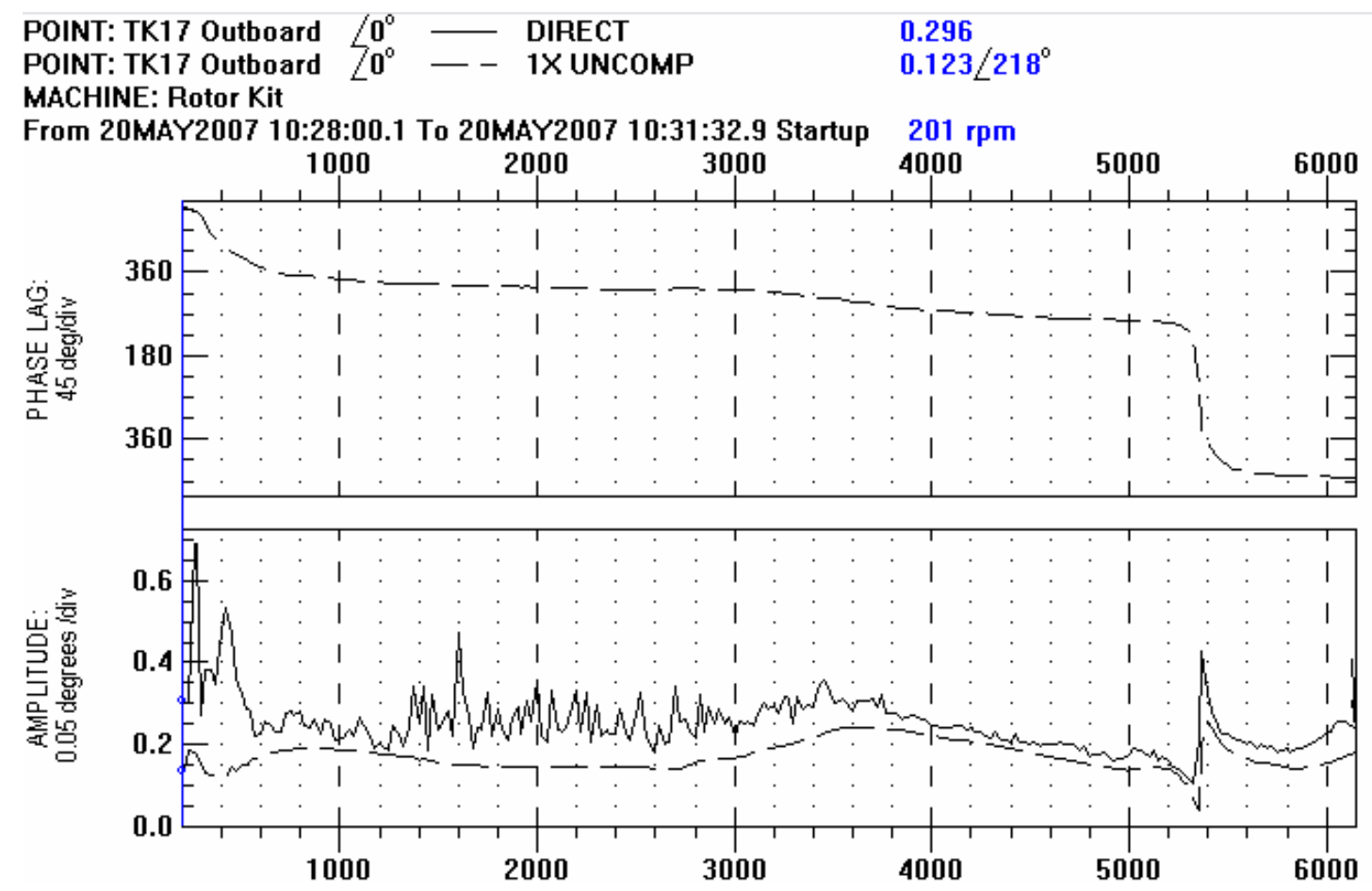

Figure 38: Torsional response of outboard disk, direct \& 1X 


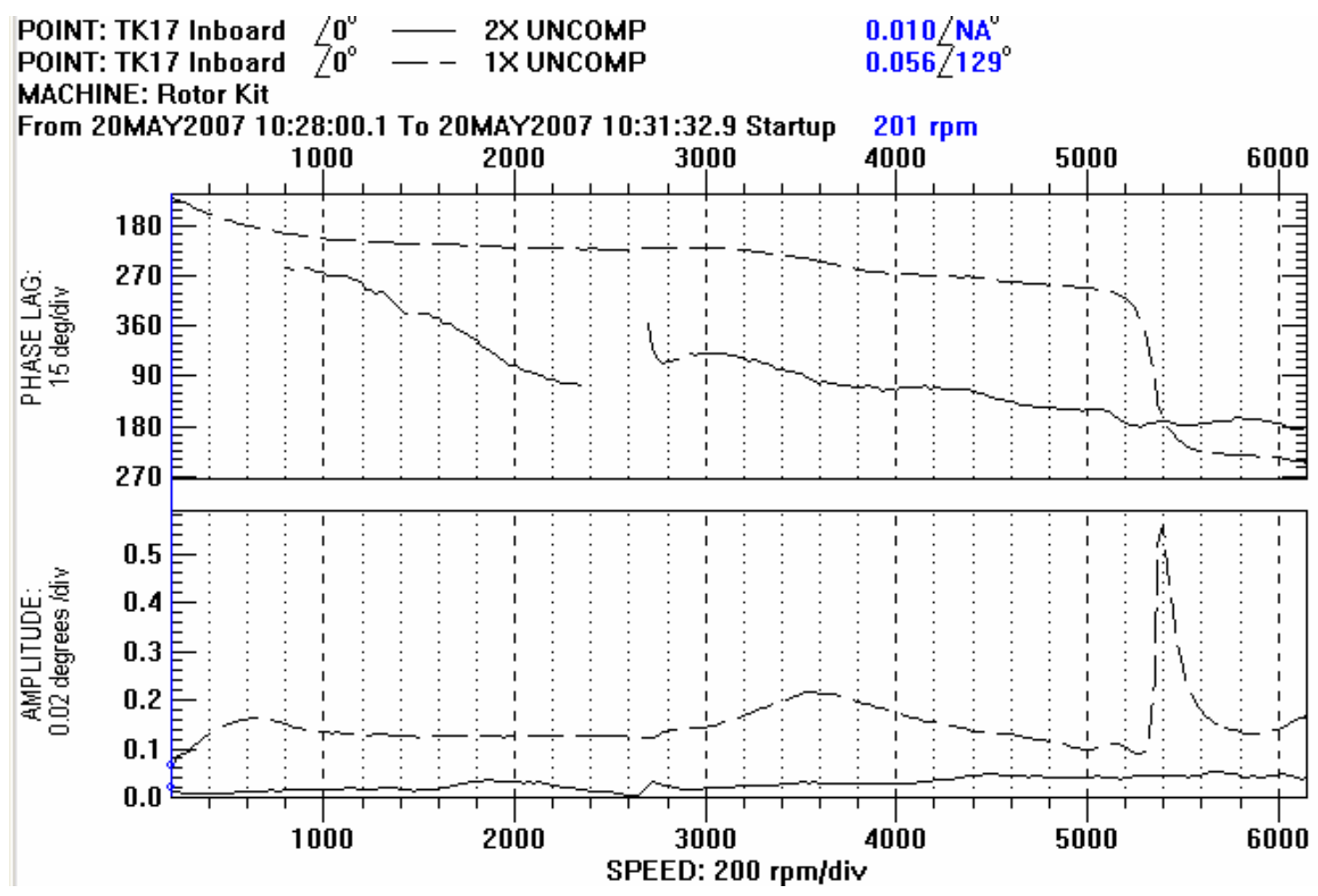

Figure 39: Overall rotor torsional response (difference between inboard \& outboard), 1X \& 2X

Additional trials were performed, but due to difficulties obtaining data from this apparatus, meaningful results were not obtained. Future experimentation should be performed to verify or refute the results of the model and simulation presented in this report. 


\section{Chapter V Conclusion}

The research presented in this paper aims to identify characteristics and trends associated with an asymmetric shaft rotor system. Understanding the behavior of a compromised rotor system is important for the early detection of failure. If trends can be identified through carefully correlated simulation, vibration experts might use those trends to prevent catastrophic, hazardous or costly failures of real machines.

This paper examines a rotor consisting of two disks connected by a torsionally flexible shaft. One disk is assumed to have rigid lateral support while the other is allowed lateral flexibility. A side load is applied to the lateral flexible disc and the shaft is considered to have asymmetric stiffness. The model mimics a real system that might consist of a turbine driving a compressor where gravitational or aerodynamic loading provides a side load and a transverse crack or similar defect results in shaft stiffness asymmetry.

Energy equations are used to derive the mathematical model proposed to describe the system. The model accounts for four degrees of freedom: rotation for both disks and translation for one. Parameters used in the mathematical model such as side loads, stiffness, damping ratios, eccentricity and natural frequencies are common to the discipline of vibration study.

The shaft stiffness model for this system assumes loads in orthogonal directions along specific axes of the shaft produce deflection only in those directions, without interaction. This model is a special case that allows exploration of the effects of reducing stiffness in one of these directions. The asymmetry factor defines the magnitude of this unidirectional reduction of stiffness. Real systems may exhibit a similar reduction of stiffness along one direction in the case of a transverse crack, for example. The asymmetry in stiffness effectively couples the torsional and lateral vibration of the rotor system.

Special cases are identified that allow simplified evaluation of the system. First, torsional vibration is assumed negligible and lateral vibration is evaluated independently. The equations of motions describing this case resemble a Jeffcott rotor with additional forcing terms related to the asymmetry. A $1 \mathrm{X}$ forcing 
term associated with imbalance is present along with $2 \mathrm{X}$ forcing terms associated with the asymmetry factor and side load. Computer simulation of this case reveals lateral response peaks at the lateral natural frequency and half the lateral natural frequency. Orbit response at half the natural frequency displays a characteristic loop-in-loop as a result of the asymmetry.

Also of interest is the case where lateral vibration is negligible and torsional vibration dominates. The equations of motion describing this case resemble a simple linear oscillator with two forcing terms. A $1 \mathrm{X}$ forcing term is associated with the unbalance, side load and asymmetry while a $2 \mathrm{X}$ forcing term is associated with the side load and asymmetry only. Computer simulation shows torsional response peaks at the torsional natural frequency and half the torsional natural frequency. An important characteristic identified in both the mathematical model and computer simulation is the response at half the torsional natural frequency grows approximately as the square of the side load while the response at the natural frequency grows approximately linearly with side load. The response amplitudes are approximately equal when the imbalance eccentricity and the displacement due to side load are equal.

When both lateral and torsional vibration and their interactions are considered, the equations of motion are more complex and the system must be characterized through parametric computer simulation. Specific studies are conducted to determine the effects of shaft asymmetry, side load, and proximity of the torsional and lateral natural frequencies. By observing changes in the response of the system resulting from changes in these parameters, conclusions can be made about their effects which may later be compared with real systems for correlation and eventually diagnosis of failure.

Shaft asymmetry in a side loaded rotor results in torsional response. As the asymmetry factor increases, torsional amplitude increases around half and one times the torsional natural frequency. Lateral vibration also begins to influence torsional vibration as the asymmetry factor increases. Simulation predicts additional torsional response at the lateral and half the lateral natural frequency as asymmetry increases. Torsional vibration should be monitored at these frequencies to alert the possibility of developing shaft asymmetry 
The effect of asymmetry on lateral response is primarily seen at half the lateral natural frequency. Here the orbit response changes distinctly from a circular to a loop-in-loop shape as asymmetry increases. Overall lateral vibration amplitude also increases with asymmetry at a higher rate at half the natural frequency than at the natural frequency. Without shaft asymmetry, no resonance is present at half the natural frequency. Torsional vibration does not appear to affect lateral vibration as in the opposite case, with no significant change in lateral response around torsional resonances.

The proximity of the torsional and lateral natural frequencies has a strong influence on the amplitude of torsional response at the torsional natural frequency. The amplitude of the torsional response at torsional resonance peaks when it corresponds to the lateral half resonance. Response at half the torsional natural frequency does not appear to be effected by proximity to lateral resonances.

Side loading impacts both torsional and lateral response. Lateral vibration is primarily affected by the $2 \mathrm{X}$ forcing term associated with side load, which causes increased lateral amplitude near half the resonant frequency. Side loading produces very little change in response at the resonant speed. Torsional response is strongly influenced by side load, especially at half the torsional natural frequency. As side load increases, lateral response at half the lateral natural frequency begins to cause torsional response at that speed as well.

In an attempt to correlate simulation with a real system, an experiment is conducted in which lateral and torsional vibration is measured for a two disc rotor with an asymmetric shaft and side load. Lateral vibration is measured with conventional proximity probes while torsional vibration is measured through gear tooth transducers and special signal processing. Some vibration characteristics are observed to correlate with simulation, notably the existence of torsional resonance and two peaks in the lateral amplitude plot. However, verification of trending is not obtained. Further experimentation should be performed. 


\section{Bibliography}

1. Wauer, J., 1990, "On the Dynamics of Cracked Rotors: A Literature Survey”, Applied Mechanics Reviews, Vol. 43(1), pp. 13-17.

2. Dimarogonas, A. D., 1996, "Vibration of Cracked Structures: A State of the Art Review," Engineering Fracture Mechanics, Vol. 55 (5), pp. 831 - 857.

3. Gasch, R., 1976, "Dynamic behavior of a simple rotor with a cross-sectional crack", Paper C178/76, I. Mech. E. Conference on Vibrations in Rotating Machinery, pp.123-128.

4. Gasch, R. A, 1993, "Survey of the Dynamic Behavior of a Simple Rotating Shaft with a Transverse Crack", Journal of Sound and Vibration Vol. 162, pp313-332.

5. Chan, R. K. C. and Lai, T. C., 1995, "Digital Simulation of a rotating shaft with a transverse Crack,” Appl. Math. Modelling Vol. 19, July pp. 411-420.

6. Mayes, I. W. and Davies, W. G. R., 1984, “Analysis of the Response of a Multi-Rotor-Bearing System Containing a Transverse Crack in a Rotor", ASME Journal of Vibration, Acoustics, Stress, and Reliability in Design, Vol. 106, pp 139-145.

7. Sawicki, J. T., Wu, X., Baaklini, G.Y. and Gyekenyesi, A., 2003, "Vibration-Based Crack Diagnosis in Rotating Shafts During Acceleration Through Resonance”, Proceedings of SPIE, Vol. 5046.

8. Sawicki, J. T., Bently, D. E., Wu, X., Baaklini, G.Y. and Friswell, M. I., 2003, "Dynamic Behavior of Cracked Flexible Rotor Subjected to Constant Driving Torque”, ISCORMA-2, Gdańsk, Poland, 4-8, August 2003, pp. 231-241.

9. Sawicki, J.T., Wu, X., Gyekenyesi, A. L. and Baaklini, G. Y., 2005 "Application of Nonlinear Dynamic Analysis for Diagnosis of Cracked Rotor Vibration Signatures", submitted to SPIE International Symposium, San Diego, California USA, March 7-10.

10. Muszynska, A., Goldman, P. and Bently, D. E., 1992, “Torsional/Lateral Cross-Coupled Responses Due to Shaft Anisotropy: A New Tool in Shaft Crack Detection”, I. Mech. E., C432090, Bath, United Kingdom, pp. 257-262. 
11. Bently, D. E., Goldman, P. and Muszynska, A., 1997, ““'Snapping” Torsional Response of an Anisotropic Radially Loaded Rotor,” Journal of Engineering for Gas Turbines and Power, Vol. 119 , pp. 397-403.

12. Wu, X., 2005, Doctoral dissertation, "Vibration-based Crack-induced Damage Detection of Shaftdisk System”, Cleveland State University.

13. Wu, X.; Meagher, J.; 2007, "Characterization of Shaft Cracks and Rotating Asymmetries using Full Spectrum and Orbit Plots”, ISCORMA-4, Calgary, Alberta, Canada, 27-31 August 2007.

14. Wu, X., Meagher, J., Judd, C.; 2007, "Investigation of coupled lateral and torsional vibrations of a cracked rotor under radial load”, IMAC XXV, Society for Experimental Mechanics, Orlando, Florida USA, February 19 -22, 2007.

15. Wu, X., Meagher, J., Volume 2008, “A Two-Disk Extended Jeffcott Rotor Model Distinguishing a Shaft Crack from Other Rotating Asymmetries", International Journal of Rotating Machinery, Article ID 846365. 


\section{Appendix A Nomenclature}

$\Theta=$ absolute angular displacement of inboard disk

$\theta=$ angular displacement of inboard disk with respect to input shaft (vibration angle)

$\Phi=$ absolute angular displacement of outboard disk

$\varphi=$ angular displacement of outboard disk with respect to input shaft (vibration angle)

$\delta=$ angle between weak axis and mass center

$\mathrm{X}=$ absolute horizontal displacement of outboard disk

$\mathrm{X}_{\mathrm{M}}=$ dynamic horizontal displacement of outboard disk

$\mathrm{Y}=$ absolute vertical displacement of outboard disk

$\mathrm{Y}_{\mathrm{M}}=$ dynamic vertical displacement of outboard disk

$\varepsilon=$ unbalance eccentricty

$\Omega=$ input shaft rotative speed

$\mathrm{P}_{\mathrm{X}}=$ horizontal load

$\mathrm{P}_{\mathrm{Y}}=$ vertical load

$\mathrm{k}_{\xi}=$ lateral shaft stiffness in weak direction

$\mathrm{k}_{\eta}=$ lateral shaft stiffness in strong direction

$\mathrm{k}=$ average shaft lateral stiffness

$q=$ stiffness asymetry factor

$\mathrm{k}_{\mathrm{t}}=$ shaft torsional stiffness

$\mathrm{k}_{\mathrm{c}}=$ coupling torsional stiffness

$\mathrm{I}_{0}=$ rotational inertia of inboard disk

$\mathrm{I}=$ rotational inertia of outboard disk

$\rho=$ radius of gyration

$\mathrm{M}=$ mass of outboard disk

$\omega_{n}=$ lateral natural frequency

$\omega_{t}=$ torsional natural frequency

$C=$ lateral damping coefficient

$C_{t}=$ shaft torsional damping coefficient

$C_{c}=$ coupling torsional damping coefficient

$\varsigma=$ lateral damping ratio

$\varsigma_{t}=$ torsional damping ratio 


\section{Appendix B Detailed Calculations}

\section{B.1 Lateral stiffness of axis-symmetric shaft}

Shaft stiffness is conveniently described in a rotating coordinate system

$$
\begin{aligned}
& {\left[\begin{array}{c}
F_{\xi} \\
F_{\eta}
\end{array}\right]=\left[\begin{array}{cc}
k_{\xi} & 0 \\
0 & k_{\eta}
\end{array}\right]\left[\begin{array}{l}
\xi \\
\eta
\end{array}\right]} \\
& K_{R}=\left[\begin{array}{cc}
k_{\xi} & 0 \\
0 & k_{\eta}
\end{array}\right]
\end{aligned}
$$

in an inertial coordinate system, the stiffness matrix would be

$$
\begin{aligned}
& {\left[\begin{array}{l}
F_{X} \\
F_{Y}
\end{array}\right]=\left[\begin{array}{ll}
k_{11} & k_{12} \\
k_{21} & k_{22}
\end{array}\right]\left[\begin{array}{l}
X \\
Y
\end{array}\right]} \\
& K_{I}=\left[\begin{array}{ll}
k_{11} & k_{12} \\
k_{21} & k_{22}
\end{array}\right]
\end{aligned}
$$

perform a coordinate transformation to determine the values of the inertial stiffness matrix

$$
\begin{aligned}
K_{I} & =T K_{R} T^{-1} \\
& =\left[\begin{array}{cc}
\cos \Phi & -\sin \Phi \\
\sin \Phi & \cos \Phi
\end{array}\right]\left[\begin{array}{cc}
k_{\xi} & 0 \\
0 & k_{\eta}
\end{array}\right]\left[\begin{array}{cc}
\cos \Phi & \sin \Phi \\
-\sin \Phi & \cos \Phi
\end{array}\right] \\
& =\left[\begin{array}{cc}
\cos \Phi & -\sin \Phi \\
\sin \Phi & \cos \Phi
\end{array}\right]\left[\begin{array}{cc}
k_{\xi} \cos \Phi & k_{\xi} \sin \Phi \\
-k_{\eta} \sin \Phi & k_{\eta} \cos \Phi
\end{array}\right] \\
& =\left[\begin{array}{ll}
k_{11} & k_{12} \\
k_{21} & k_{22}
\end{array}\right]
\end{aligned}
$$




$$
\begin{aligned}
k_{11} & =k_{\xi} \cos ^{2} \Phi+k_{\eta} \sin ^{2} \Phi \\
& =k_{\xi}\left(\frac{1+\cos 2 \Phi}{2}\right)+k_{\eta}\left(\frac{1-\cos 2 \Phi}{2}\right) \\
& =\left(\frac{k_{\xi}+k_{\eta}}{2}\right)-\left(\frac{k_{\eta}-k_{\xi}}{2}\right) \cos 2 \Phi \\
& =k-k q \cos 2 \Phi \\
& =k(1-q \cos 2 \Phi) \\
k_{12} & =k_{\xi} \cos \Phi \sin \Phi-k_{\eta} \cos \Phi \sin \Phi \\
& =-\left(\frac{k_{\eta}-k_{\xi}}{2}\right) \sin 2 \Phi \\
& =-k q \sin 2 \Phi \\
& =k+k q \cos 2 \Phi \\
= & k(1+q \cos 2 \Phi) \\
& =\left(\frac{k_{\xi}+k_{\eta}}{2}\right)+\left(\frac{k_{\eta}-k_{\xi}}{2}\right) \cos 2 \Phi \\
k_{22} & =k_{\xi} \sin { }^{2} \Phi+k_{\eta} \cos ^{2} \Phi \\
& =k_{21}
\end{aligned}
$$

thus, the stiffness matrix in inertial coordinates is given by

$$
K_{I}=k\left[\begin{array}{cc}
1-q \cos 2 \Phi & -q \sin 2 \Phi \\
-q \sin 2 \Phi & 1+q \cos 2 \Phi
\end{array}\right]
$$




\section{B.2 Geometric Relationships}

$\Phi=\Omega t+\varphi-\Phi_{0}$

$\dot{\Phi}=\Omega+\dot{\varphi}$

$\ddot{\Phi}=\ddot{\varphi}$

$\Theta=\Omega t+\theta-\Theta_{0}$

$\dot{\Theta}=\Omega+\dot{\theta}$

$\ddot{\Theta}=\ddot{\theta}$

$x_{c m}=X+\varepsilon \cos (\Phi+\delta)$

$\dot{x}_{c m}=\dot{X}-\varepsilon \dot{\Phi} \sin (\Phi+\delta)$

$y_{c m}=Y+\varepsilon \sin (\Phi+\delta)$

$\dot{y}_{c m}=\dot{Y}+\varepsilon \dot{\Phi} \cos (\Phi+\delta)$

assuming lateral vibration is about the static offset due to side loading

$X=X_{M}-\frac{P_{X}}{k}$

$X=X_{M}-\frac{1}{\omega_{n}^{2}}\left(\frac{P_{X}}{M}\right)$

$\dot{X}=\dot{X}_{M}$

$\ddot{X}=\ddot{X}_{M}$

$Y=Y_{M}-\frac{P_{Y}}{k}$

$Y=Y_{M}-\frac{1}{\omega_{n}^{2}}\left(\frac{P_{Y}}{M}\right)$

$\dot{Y}=\dot{Y}_{M}$

$\ddot{Y}=\ddot{Y}_{M}$

\section{B.3 Other Relationships \& Substitutions}




$$
k=\frac{k_{\xi}+k_{\eta}}{2}, q=\frac{k_{\eta}-k_{\xi}}{2 k}, \omega_{n}=\sqrt{\frac{k}{M}}, \omega_{t}=\sqrt{\frac{k_{t}}{I}}, \varsigma=\frac{C}{\sqrt{k M}}, \varsigma_{t}=\frac{C_{t}}{\sqrt{k_{t} I}}, I=\rho^{2} M, \tau=\omega_{n} t
$$

\section{B.4 Axis-symmetric Shaft Stiffness}

shaft stiffness is conveniently described in a rotating coordinate system

$$
\begin{aligned}
& {\left[\begin{array}{l}
F_{\xi} \\
F_{\eta}
\end{array}\right]=\left[\begin{array}{cc}
k_{\xi} & 0 \\
0 & k_{\eta}
\end{array}\right]\left[\begin{array}{l}
\xi \\
\eta
\end{array}\right]} \\
& K_{R}=\left[\begin{array}{cc}
k_{\xi} & 0 \\
0 & k_{\eta}
\end{array}\right]
\end{aligned}
$$

in an inertial coordinate system, the stiffness matrix would be

$$
\begin{aligned}
& {\left[\begin{array}{l}
F_{X} \\
F_{Y}
\end{array}\right]=\left[\begin{array}{ll}
k_{11} & k_{12} \\
k_{21} & k_{22}
\end{array}\right]\left[\begin{array}{l}
X \\
Y
\end{array}\right]} \\
& K_{I}=\left[\begin{array}{ll}
k_{11} & k_{12} \\
k_{21} & k_{22}
\end{array}\right]
\end{aligned}
$$

perform a coordinate transformation to determine the values of the inertial stiffness matrix

$$
\begin{aligned}
K_{I} & =T K_{R} T^{-1} \\
& =\left[\begin{array}{cc}
\cos \Phi & -\sin \Phi \\
\sin \Phi & \cos \Phi
\end{array}\right]\left[\begin{array}{cc}
k_{\xi} & 0 \\
0 & k_{\eta}
\end{array}\right]\left[\begin{array}{cc}
\cos \Phi & \sin \Phi \\
-\sin \Phi & \cos \Phi
\end{array}\right] \\
& =\left[\begin{array}{cc}
\cos \Phi & -\sin \Phi \\
\sin \Phi & \cos \Phi
\end{array}\right]\left[\begin{array}{cc}
k_{\xi} \cos \Phi & k_{\xi} \sin \Phi \\
-k_{\eta} \sin \Phi & k_{\eta} \cos \Phi
\end{array}\right] \\
& =\left[\begin{array}{ll}
k_{11} & k_{12} \\
k_{21} & k_{22}
\end{array}\right]
\end{aligned}
$$




$$
\begin{aligned}
k_{11} & =k_{\xi} \cos ^{2} \Phi+k_{\eta} \sin ^{2} \Phi \\
& =k_{\xi}\left(\frac{1+\cos 2 \Phi}{2}\right)+k_{\eta}\left(\frac{1-\cos 2 \Phi}{2}\right) \\
& =\left(\frac{k_{\xi}+k_{\eta}}{2}\right)-\left(\frac{k_{\eta}-k_{\xi}}{2}\right) \cos 2 \Phi \\
& =k-k q \cos 2 \Phi \\
& =k(1-q \cos 2 \Phi)
\end{aligned}
$$

$$
\begin{aligned}
k_{12} & =k_{\xi} \cos \Phi \sin \Phi-k_{\eta} \cos \Phi \sin \Phi \\
& =-\left(\frac{k_{\eta}-k_{\xi}}{2}\right) \sin 2 \Phi \\
& =-k q \sin 2 \Phi
\end{aligned}
$$

$$
\begin{aligned}
k_{12} & =k_{21} \\
k_{22} & =k_{\xi} \sin ^{2} \Phi+k_{\eta} \cos ^{2} \Phi \\
& =k_{\xi}\left(\frac{1-\cos 2 \Phi}{2}\right)+k_{\eta}\left(\frac{1+\cos 2 \Phi}{2}\right) \\
& =\left(\frac{k_{\xi}+k_{\eta}}{2}\right)+\left(\frac{k_{\eta}-k_{\xi}}{2}\right) \cos 2 \Phi \\
& =k+k q \cos 2 \Phi \\
& =k(1+q \cos 2 \Phi)
\end{aligned}
$$

thus, the stiffness matrix in inertial coordinates is given by

$$
K_{I}=k\left[\begin{array}{cc}
1-q \cos 2 \Phi & -q \sin 2 \Phi \\
-q \sin 2 \Phi & 1+q \cos 2 \Phi
\end{array}\right]
$$




\section{B.5 Detailed Derivation}

Kinetic Energy of the System

$$
\begin{aligned}
T & =\frac{1}{2} I \dot{\Phi}^{2}+\frac{1}{2} I_{0} \dot{\Theta}^{2}+\frac{1}{2} M \dot{x}_{c m}{ }^{2}+\frac{1}{2} M \dot{y}_{c m}{ }^{2} \\
& =\frac{1}{2} I \dot{\Phi}^{2}+\frac{1}{2} I_{0} \dot{\Theta}^{2}+\frac{1}{2} M\left\{[\dot{X}-\varepsilon \dot{\Phi} \sin (\Phi+\delta)]^{2}+[\dot{Y}+\varepsilon \dot{\Phi} \cos (\Phi+\delta)]^{2}\right\} \\
& =\frac{1}{2} I \dot{\Phi}^{2}+\frac{1}{2} I_{0} \dot{\Theta}^{2}+\frac{1}{2} M\left\{\dot{X}^{2}+\dot{Y}^{2}+2 \varepsilon \dot{\Phi}[\dot{Y} \cos (\Phi+\delta)-\dot{X} \sin (\Phi+\delta)]+\varepsilon^{2} \dot{\Phi}^{2}\right\}
\end{aligned}
$$

Prepare for Lagrange Equations for X, Y, $\Phi$ and $\Theta$

$$
\begin{aligned}
& \frac{\partial T}{\partial X}=0 \\
& \frac{\partial T}{\partial \dot{X}}=M \dot{X}-\varepsilon M \dot{\Phi} \sin (\Phi+\delta) \\
& \frac{\partial}{\partial t}\left(\frac{\partial T}{\partial \dot{X}}\right)=M \ddot{X}-\varepsilon M \ddot{\Phi} \sin (\Phi+\delta)-\varepsilon M \dot{\Phi}^{2} \cos (\Phi+\delta)
\end{aligned}
$$

$$
\begin{aligned}
& \frac{\partial T}{\partial Y}=0 \\
& \frac{\partial T}{\partial \dot{Y}}=M \dot{Y}+\varepsilon M \dot{\Phi} \cos (\Phi+\delta) \\
& \frac{\partial}{\partial t}\left(\frac{\partial T}{\partial \dot{Y}}\right)=M \ddot{Y}+\varepsilon M \ddot{\Phi} \cos (\Phi+\delta)-\varepsilon M \dot{\Phi}^{2} \sin (\Phi+\delta)
\end{aligned}
$$

$$
\begin{aligned}
& \frac{\partial T}{\partial \Theta}=0 \\
& \frac{\partial T}{\partial \dot{\Theta}}=I_{0} \dot{\Theta} \\
& \frac{\partial}{\partial t}\left(\frac{\partial T}{\partial \dot{\Theta}}\right)=I_{0} \ddot{\Theta}
\end{aligned}
$$




$$
\begin{aligned}
& \frac{\partial T}{\partial \Phi}=-\varepsilon M \dot{\Phi}[\dot{X} \cos (\Phi+\delta)+\dot{Y} \sin (\Phi+\delta)] \\
& \frac{\partial T}{\partial \dot{\Phi}}=\left(I+\varepsilon^{2} M\right) \dot{\Phi}+\varepsilon M[\dot{Y} \cos (\Phi+\delta)-\dot{X} \sin (\Phi+\delta)] \\
& \frac{\partial}{\partial t}\left(\frac{\partial T}{\partial \dot{\Phi}}\right)=\left(I+\varepsilon^{2} M\right) \ddot{\Phi}+\varepsilon M[(\ddot{Y}-\dot{X} \dot{\Phi}) \cos (\Phi+\delta)-(\ddot{X}+\dot{Y} \dot{\Phi}) \sin (\Phi+\delta)]
\end{aligned}
$$

Potential Energy of the System

$$
\begin{aligned}
& U=\frac{1}{2}\left[\begin{array}{ll}
X & Y
\end{array}\right]\left[\begin{array}{ll}
k_{11} & k_{12} \\
k_{21} & k_{22}
\end{array}\right]\left[\begin{array}{l}
X \\
Y
\end{array}\right]+\frac{1}{2} k_{t}(\Phi-\Theta)^{2} \\
& k_{12}=k_{21} \\
& \therefore U=\frac{1}{2}\left(k_{11} X^{2}+k_{22} Y^{2}\right)+k_{12} X Y+\frac{1}{2} k_{t}(\Phi-\Theta)^{2}
\end{aligned}
$$

for an axis-symmetric shaft

$$
U=\frac{1}{2} k\left[(1-q \cos 2 \Phi) X^{2}+(1+q \cos 2 \Phi) Y^{2}\right]-k q \sin 2 \Phi X Y+\frac{1}{2} k_{t}(\Phi-\Theta)^{2}
$$

Prepare for Lagrange Equations for X, Y, $\Phi$ and $\Theta$

$$
\begin{aligned}
& \frac{\partial U}{\partial X}=k(1-q \cos 2 \Phi) X-k q \sin 2 \Phi Y \\
& \frac{\partial U}{\partial Y}=k(1+q \cos 2 \Phi) Y-k q \sin 2 \Phi X \\
& \frac{\partial U}{\partial \Phi}=k q \sin 2 \Phi\left(X^{2}-Y^{2}\right)-2 k q \cos 2 \Phi X Y+k_{t}(\Phi-\Theta) \\
& \frac{\partial U}{\partial \Theta}=-k_{t}(\Phi-\Theta)
\end{aligned}
$$


Dissipation Function of the System

$$
D=\frac{1}{2} C \dot{X}^{2}+\frac{1}{2} C \dot{Y}^{2}+\frac{1}{2} C_{t}(\dot{\Phi}-\dot{\Theta})^{2}
$$

Prepare for Lagrange Equations for X, Y, $\Phi$ and $\Theta$

$$
\begin{aligned}
& \frac{\partial D}{\partial \dot{X}}=C \dot{X} \\
& \frac{\partial D}{\partial \dot{Y}}=C \dot{Y} \\
& \frac{\partial D}{\partial \dot{\Phi}}=C_{t}(\dot{\Phi}-\dot{\Theta}) \\
& \frac{\partial D}{\partial \dot{\Theta}}=-C_{t}(\dot{\Phi}-\dot{\Theta})
\end{aligned}
$$

Lagrange Equation for X 


$$
\begin{aligned}
& \frac{\partial}{\partial t}\left(\frac{\partial T}{\partial \dot{X}}\right)-\frac{\partial T}{\partial X}+\frac{\partial U}{\partial X}+\frac{\partial D}{\partial \dot{X}}=-P_{X} \\
& M \ddot{X}-\varepsilon M \ddot{\Phi} \sin (\Phi+\delta)-\varepsilon M \dot{\Phi}^{2} \cos (\Phi+\delta)+k(1-q \cos 2 \Phi) X-k q \sin 2 \Phi Y+C \dot{X}=-P_{X} \\
& \ddot{X}-\varepsilon \ddot{\Phi} \sin (\Phi+\delta)-\varepsilon \dot{\Phi}^{2} \cos (\Phi+\delta)+\omega_{n}^{2}(1-q \cos 2 \Phi) X-\omega_{n}^{2} q \sin 2 \Phi Y+2 \varsigma \omega_{n} \dot{X}=-\frac{P_{X}}{M} \\
& \ddot{X}+2 \varsigma \omega_{n} \dot{X}+\omega_{n}^{2}(1-q \cos 2 \Phi) X=\varepsilon \ddot{\Phi} \sin (\Phi+\delta)+\varepsilon \dot{\Phi}^{2} \cos (\Phi+\delta)+\omega_{n}^{2} q \sin 2 \Phi Y-\frac{P_{X}}{M}
\end{aligned}
$$

substituting

$$
\begin{aligned}
& X=X_{M}-\frac{1}{\omega_{n}^{2}}\left(\frac{P_{X}}{M}\right), \dot{X}=\dot{X}_{M}, \ddot{X}=\ddot{X}_{M} \quad Y=Y_{M}-\frac{1}{\omega_{n}^{2}}\left(\frac{P_{Y}}{M}\right), \dot{Y}=\dot{Y}_{M}, \ddot{Y}=\ddot{Y}_{M} \\
& \ddot{X}_{M}+2 \varsigma \omega_{n} \dot{X}_{M}+\omega_{n}^{2}(1-q \cos 2 \Phi)\left[X_{M}-\frac{1}{\omega_{n}^{2}}\left(\frac{P_{X}}{M}\right)\right] \\
& =\varepsilon \ddot{\Phi} \sin (\Phi+\delta)+\varepsilon \dot{\Phi}^{2} \cos (\Phi+\delta)+\omega_{n}^{2} q \sin 2 \Phi\left[Y_{M}-\frac{1}{\omega_{n}^{2}}\left(\frac{P_{Y}}{M}\right)\right]-\frac{P_{X}}{M} \\
& \ddot{X}_{M}+2 \varsigma \omega_{n} \dot{X}_{M}+\omega_{n}^{2}(1-q \cos 2 \Phi) X_{M}-\omega_{n}^{2} q \sin 2 \Phi Y_{M} \\
& =\varepsilon \ddot{\Phi} \sin (\Phi+\delta)+\varepsilon \dot{\Phi}^{2} \cos (\Phi+\delta)-\left(\frac{P_{Y}}{M}\right) q \sin 2 \Phi-\left(\frac{P_{X}}{M}\right) q \cos 2 \Phi
\end{aligned}
$$

substituting $\Phi=\Omega t+\varphi-\Phi_{0}, \dot{\Phi}=\Omega+\dot{\varphi}, \ddot{\Phi}=\ddot{\varphi}$

$$
\begin{aligned}
& \ddot{X}_{M}+2 \varsigma \omega_{n} \dot{X}_{M}+\omega_{n}^{2}\left(1-q \cos \left(2\left(\Omega t+\varphi-\Phi_{0}\right)\right)\right) X_{M}-\omega_{n}^{2} q \sin \left(2\left(\Omega t+\varphi-\Phi_{0}\right)\right) Y_{M}=\varepsilon \ddot{\varphi} \sin \left(\Omega t+\varphi-\Phi_{0}+\delta\right) \\
& +\varepsilon(\Omega+\dot{\varphi})^{2} \cos \left(\Omega t+\varphi-\Phi_{0}+\delta\right)-\left(\frac{P_{Y}}{M}\right) q \sin \left(2\left(\Omega t+\varphi-\Phi_{0}\right)\right)-\left(\frac{P_{X}}{M}\right) q \cos \left(2\left(\Omega t+\varphi-\Phi_{0}\right)\right) \\
& { }_{\text {let }} \tau=\omega_{n} t, \frac{d(\square)}{d t}=\omega_{n} \frac{d(\square)}{d \tau}=\omega_{n}(\square)^{\prime}, \frac{d^{2}(\square)}{d t^{2}}=\omega_{n}^{2} \frac{d^{2}(\square)}{d \tau^{2}}=\omega_{n}^{2}(\square)^{\prime \prime}
\end{aligned}
$$




$$
\begin{aligned}
& X_{M}^{\prime \prime}+2 \varsigma X_{M}^{\prime}+\left(1-q \cos \left(2\left(\frac{\Omega}{\omega_{n}} \tau+\varphi-\Phi_{0}\right)\right)\right) X_{M}-q \sin \left(2\left(\frac{\Omega}{\omega_{n}} \tau+\varphi-\Phi_{0}\right)\right) Y_{M}=\varepsilon \varphi^{\prime \prime} \sin \left(\frac{\Omega}{\omega_{n}} \tau+\varphi-\Phi_{0}+\delta\right) \\
& +\frac{\varepsilon}{\omega_{n}^{2}}\left(\Omega+\omega_{n} \varphi^{\prime}\right)^{2} \cos \left(\frac{\Omega}{\omega_{n}} \tau+\varphi-\Phi_{0}+\delta\right)-\left(\frac{P_{Y}}{M}\right) \frac{q}{\omega_{n}^{2}} \sin \left(2\left(\frac{\Omega}{\omega_{n}} \tau+\varphi-\Phi_{0}\right)\right)-\left(\frac{P_{X}}{M}\right) \frac{q}{\omega_{n}^{2}} \cos \left(2\left(\frac{\Omega}{\omega_{n}} \tau+\varphi-\Phi_{0}\right)\right)
\end{aligned}
$$

Lagrange Equation for $\mathrm{Y}$

$$
\begin{aligned}
& \frac{\partial}{\partial t}\left(\frac{\partial T}{\partial \dot{Y}}\right)-\frac{\partial T}{\partial Y}+\frac{\partial U}{\partial Y}+\frac{\partial D}{\partial \dot{Y}}=-P_{Y} \\
& M \ddot{Y}+\varepsilon M \ddot{\Phi} \cos (\Phi+\delta)-\varepsilon M \dot{\Phi}^{2} \sin (\Phi+\delta)+k(1+q \cos 2 \Phi) Y-k q \sin 2 \Phi X+C \dot{Y}=-P_{Y} \\
& \ddot{Y}+\varepsilon \ddot{\Phi} \cos (\Phi+\delta)-\varepsilon \dot{\Phi}^{2} \sin (\Phi+\delta)+\omega_{n}^{2}(1+q \cos 2 \Phi) Y-\omega_{n}^{2} q \sin 2 \Phi X+2 \varsigma \omega_{n} \dot{Y}=-\frac{P_{Y}}{M} \\
& \ddot{Y}+2 \varsigma \omega_{n} \dot{Y}+\omega_{n}^{2}(1+q \cos 2 \Phi) Y=-\varepsilon \ddot{\Phi} \cos (\Phi+\delta)+\varepsilon \dot{\Phi}^{2} \sin (\Phi+\delta)+\omega_{n}^{2} q \sin 2 \Phi X-\frac{P_{Y}}{M}
\end{aligned}
$$

substituting

$$
\begin{aligned}
& X=X_{M}-\frac{1}{\omega_{n}^{2}}\left(\frac{P_{X}}{M}\right), \dot{X}=\dot{X}_{M}, \ddot{X}=\ddot{X}_{M} Y=Y_{M}-\frac{1}{\omega_{n}^{2}}\left(\frac{P_{Y}}{M}\right), \dot{Y}=\dot{Y}_{M}, \ddot{Y}=\ddot{Y}_{M} \\
& \ddot{Y}_{M}+2 \varsigma \omega_{n} \dot{Y}_{M}+\omega_{n}^{2}(1+q \cos 2 \Phi)\left[Y_{M}-\frac{1}{\omega_{n}^{2}}\left(\frac{P_{Y}}{M}\right)\right] \\
& =-\varepsilon \ddot{\Phi} \cos (\Phi+\delta)+\varepsilon \dot{\Phi}^{2} \sin (\Phi+\delta)+\omega_{n}^{2} q \sin 2 \Phi\left[X_{M}-\frac{1}{\omega_{n}^{2}}\left(\frac{P_{X}}{M}\right)\right]-\frac{P_{Y}}{M} \\
& \ddot{Y}_{M}+2 \varsigma \omega_{n} \dot{Y}_{M}+\omega_{n}^{2}(1+q \cos 2 \Phi) Y_{M}-\omega_{n}^{2} q \sin 2 \Phi X_{M} \\
& =-\varepsilon \ddot{\Phi} \cos (\Phi+\delta)+\varepsilon \dot{\Phi}^{2} \sin (\Phi+\delta)-\left(\frac{P_{X}}{M}\right) q \sin 2 \Phi+\left(\frac{P_{Y}}{M}\right) q \cos 2 \Phi
\end{aligned}
$$

substituting $\Phi=\Omega t+\varphi-\Phi_{0}, \dot{\Phi}=\Omega+\dot{\varphi}, \ddot{\Phi}=\ddot{\varphi}$ 


$$
\begin{aligned}
& \ddot{Y}_{M}+2 \varsigma \omega_{n} \dot{Y}_{M}+\omega_{n}^{2}\left(1+q \cos \left(2\left(\Omega t+\varphi-\Phi_{0}\right)\right)\right) Y_{M}-\omega_{n}^{2} q \sin \left(2\left(\Omega t+\varphi-\Phi_{0}\right)\right) X_{M}=-\varepsilon \ddot{\varphi} \cos \left(\Omega t+\varphi-\Phi_{0}+\delta\right) \\
& +\varepsilon(\Omega+\dot{\varphi})^{2} \sin \left(\Omega t+\varphi-\Phi_{0}+\delta\right)-\left(\frac{P_{X}}{M}\right) q \sin \left(2\left(\Omega t+\varphi-\Phi_{0}\right)\right)+\left(\frac{P_{Y}}{M}\right) q \cos \left(2\left(\Omega t+\varphi-\Phi_{0}\right)\right) \\
& \text { let } \tau=\omega_{n} t, \frac{d(\square)}{d t}=\omega_{n} \frac{d(\square)}{d \tau}=\omega_{n}(\square)^{\prime} \frac{d^{2}(\square)}{d t^{2}}=\omega_{n}^{2} \frac{d^{2}(\square)}{d \tau^{2}}=\omega_{n}^{2}(\square)^{\prime \prime} \\
& Y_{M}^{\prime \prime}+2 \varsigma Y_{M}^{\prime}+\left(1+q \cos \left(2\left(\frac{\Omega}{\omega_{n}} \tau+\varphi-\Phi_{0}\right)\right)\right) Y_{M}-q \sin \left(2\left(\frac{\Omega}{\omega_{n}} \tau+\varphi-\Phi_{0}\right)\right) X_{M}=-\varepsilon \varphi^{\prime \prime} \cos \left(\frac{\Omega}{\omega_{n}} \tau+\varphi-\Phi_{0}+\delta\right) \\
& +\frac{\varepsilon}{\omega_{n}^{2}}\left(\Omega+\omega_{n} \varphi^{\prime}\right)^{2} \sin \left(\frac{\Omega}{\omega_{n}} \tau+\varphi-\Phi_{0}+\delta\right)-\left(\frac{P_{X}}{M}\right) \frac{q}{\omega_{n}^{2}} \sin \left(2\left(\frac{\Omega}{\omega_{n}} \tau+\varphi-\Phi_{0}\right)\right)+\left(\frac{P_{Y}}{M}\right) \frac{q}{\omega_{n}^{2}} \cos \left(2\left(\frac{\Omega}{\omega_{n}} \tau+\varphi-\Phi_{0}\right)\right)
\end{aligned}
$$


Lagrange Equation for $\Phi$

$\frac{\partial}{\partial t}\left(\frac{\partial T}{\partial \dot{\Phi}}\right)-\frac{\partial T}{\partial \Phi}+\frac{\partial U}{\partial \Phi}+\frac{\partial D}{\partial \dot{\Phi}}=0$

$\left(I+\varepsilon^{2} M\right) \ddot{\Phi}+\varepsilon M[(\ddot{Y}-\dot{X} \dot{\Phi}) \cos (\Phi+\delta)-(\ddot{X}+\dot{Y} \dot{\Phi}) \sin (\Phi+\delta)]$

$+\varepsilon M \dot{\Phi}[\dot{X} \cos (\Phi+\delta)+\dot{Y} \sin (\Phi+\delta)]+k q \sin 2 \Phi\left(X^{2}-Y^{2}\right)$

$-2 k q \cos 2 \Phi X Y+k_{t}(\Phi-\Theta)+C_{t}(\dot{\Phi}-\dot{\Theta})=0$

$\left(\frac{I}{M}+\varepsilon^{2}\right) \ddot{\Phi}+\varepsilon[\ddot{Y} \cos (\Phi+\delta)-\ddot{X} \sin (\Phi+\delta)]+\omega_{n}^{2} q \sin 2 \Phi\left(X^{2}-Y^{2}\right)$

$-2 \omega_{n}^{2} q \cos 2 \Phi X Y+\frac{k_{t}}{M}(\Phi-\Theta)+\frac{C_{t}}{M}(\dot{\Phi}-\dot{\Theta})=0$

$\left(\rho^{2}+\varepsilon^{2}\right) \ddot{\Phi}+\varepsilon[\ddot{Y} \cos (\Phi+\delta)-\ddot{X} \sin (\Phi+\delta)]+\omega_{n}^{2} q \sin 2 \Phi\left(X^{2}-Y^{2}\right)$

$-2 \omega_{n}^{2} q \cos 2 \Phi X Y+\frac{\rho^{2} k_{t}}{I}(\Phi-\Theta)+\frac{\rho^{2} C_{t}}{I}(\dot{\Phi}-\dot{\Theta})=0$

$\left(\rho^{2}+\varepsilon^{2}\right) \ddot{\Phi}+\varepsilon[\ddot{Y} \cos (\Phi+\delta)-\ddot{X} \sin (\Phi+\delta)]+\omega_{n}^{2} q \sin 2 \Phi\left(X^{2}-Y^{2}\right)$

$-2 \omega_{n}^{2} q \cos 2 \Phi X Y+\rho^{2} \omega_{t}^{2}(\Phi-\Theta)+2 \rho^{2} \varsigma_{t} \omega_{t}(\dot{\Phi}-\dot{\Theta})=0$ 
from Lagrange equation for Y,

$\ddot{Y} \cos (\Phi+\delta)=-2 \varsigma \omega_{n} \cos (\Phi+\delta) \dot{Y}-\omega_{n}^{2}(1+q \cos 2 \Phi) \cos (\Phi+\delta) Y$

$-\varepsilon \ddot{\Phi} \cos ^{2}(\Phi+\delta)+\varepsilon \dot{\Phi}^{2} \cos (\Phi+\delta) \sin (\Phi+\delta)+\omega_{n}^{2} q \sin 2 \Phi \cos (\Phi+\delta) X-\frac{P_{Y}}{M} \cos (\Phi+\delta)$

from Lagrange equation for X,

$\ddot{X} \sin (\Phi+\delta)=-2 \varsigma \omega_{n} \sin (\Phi+\delta) \dot{X}-\omega_{n}^{2}(1-q \cos 2 \Phi) \sin (\Phi+\delta) X$

$+\varepsilon \ddot{\Phi} \sin ^{2}(\Phi+\delta)+\varepsilon \dot{\Phi}^{2} \cos (\Phi+\delta) \sin (\Phi+\delta)+\omega_{n}^{2} q \sin 2 \Phi \sin (\Phi+\delta) Y-\frac{P_{X}}{M} \sin (\Phi+\delta)$

it follows that 


$$
\begin{aligned}
& \ddot{Y} \cos (\Phi+\delta)-\ddot{X} \sin (\Phi+\delta)=2 \varsigma \omega_{n}[\sin (\Phi+\delta) \dot{X}-\cos (\Phi+\delta) \dot{Y}] \\
& +\omega_{n}^{2}(1-q \cos 2 \Phi) \sin (\Phi+\delta) X-\omega_{n}^{2}(1+q \cos 2 \Phi) \cos (\Phi+\delta) Y \\
& -\varepsilon \ddot{\Phi}+\omega_{n}^{2} q \sin 2 \Phi[\cos (\Phi+\delta) X-\sin (\Phi+\delta) Y]+\frac{P_{X}}{M} \sin (\Phi+\delta)-\frac{P_{Y}}{M} \cos (\Phi+\delta) \\
& \ddot{Y} \cos (\Phi+\delta)-\ddot{X} \sin (\Phi+\delta)=2 \varsigma \omega_{n}[\sin (\Phi+\delta) \dot{X}-\cos (\Phi+\delta) \dot{Y}] \\
& +\omega_{n}^{2}[\sin (\Phi+\delta) X-\cos (\Phi+\delta) Y] \\
& +\omega_{n}^{2} q[\sin 2 \Phi \cos (\Phi+\delta)-\cos 2 \Phi \sin (\Phi+\delta)] X \\
& -\omega_{n}^{2} q[\cos 2 \Phi \cos (\Phi+\delta)+\sin 2 \Phi \sin (\Phi+\delta)] Y \\
& +\frac{P_{X}}{M} \sin (\Phi+\delta)-\frac{P_{Y}}{M} \cos (\Phi+\delta)-\varepsilon \ddot{\Phi} \\
& \ddot{Y} \cos (\Phi+\delta)-\ddot{X} \sin (\Phi+\delta)=2 \varsigma \omega_{n}[\sin (\Phi+\delta) \dot{X}-\cos (\Phi+\delta) \dot{Y}] \\
& +\omega_{n}^{2}[\sin (\Phi+\delta) X-\cos (\Phi+\delta) Y]+\omega_{n}^{2} q[\sin (\Phi-\delta) X-\cos (\Phi-\delta) Y] \\
& +\frac{P_{X}}{M} \sin (\Phi+\delta)-\frac{P_{Y}}{M} \cos (\Phi+\delta)-\varepsilon \ddot{\Phi}
\end{aligned}
$$


substituting into Lagrange equation for $\Phi$

$$
\begin{aligned}
& \left(\rho^{2}+\varepsilon^{2}\right) \ddot{\Phi}+\varepsilon 2 \varsigma \omega_{n}[\sin (\Phi+\delta) \dot{X}-\cos (\Phi+\delta) \dot{Y}] \\
& +\varepsilon \omega_{n}^{2}[\sin (\Phi+\delta) X-\cos (\Phi+\delta) Y]+\varepsilon \omega_{n}^{2} q[\sin (\Phi-\delta) X-\cos (\Phi-\delta) Y] \\
& +\varepsilon \frac{P_{X}}{M} \sin (\Phi+\delta)-\varepsilon \frac{P_{Y}}{M} \cos (\Phi+\delta)-\varepsilon^{2} \ddot{\Phi}+\omega_{n}^{2} q \sin 2 \Phi\left(X^{2}-Y^{2}\right) \\
& -2 \omega_{n}^{2} q \cos 2 \Phi X Y+\rho^{2} \omega_{t}^{2}(\Phi-\Theta)+2 \rho^{2} \varsigma_{t} \omega_{t}(\dot{\Phi}-\dot{\Theta})=0 \\
& \rho^{2} \ddot{\Phi}+\varepsilon 2 \varsigma \omega_{n}[\sin (\Phi+\delta) \dot{X}-\cos (\Phi+\delta) \dot{Y}] \\
& +\varepsilon \omega_{n}^{2}[\sin (\Phi+\delta) X-\cos (\Phi+\delta) Y]+\varepsilon \omega_{n}^{2} q[\sin (\Phi-\delta) X-\cos (\Phi-\delta) Y] \\
& +\varepsilon \frac{P_{X}}{M} \sin (\Phi+\delta)-\varepsilon \frac{P_{Y}}{M} \cos (\Phi+\delta)+\omega_{n}^{2} q\left[\sin 2 \Phi\left(X^{2}-Y^{2}\right)-2 \cos 2 \Phi X Y\right] \\
& +\rho^{2} \omega_{t}^{2}(\Phi-\Theta)+2 \rho^{2} \varsigma_{t} \omega_{t}(\dot{\Phi}-\dot{\Theta})=0 \\
& \ddot{\Phi}+\omega_{t}^{2}(\Phi-\Theta)+2 \varsigma_{t} \omega_{t}(\dot{\Phi}-\dot{\Theta})=\frac{\varepsilon 2 \varsigma \omega_{n}}{\rho^{2}}[\cos (\Phi+\delta) \dot{Y}-\sin (\Phi+\delta) \dot{X}] \\
& +\frac{\varepsilon \omega_{n}^{2}}{\rho^{2}}[\cos (\Phi+\delta) Y-\sin (\Phi+\delta) X]+\frac{\varepsilon \omega_{n}^{2} q}{\rho^{2}}[\cos (\Phi-\delta) Y-\sin (\Phi-\delta) X] \\
& +\frac{\varepsilon}{\rho^{2}}\left[\left(\frac{P_{Y}}{M}\right) \cos (\Phi+\delta)-\left(\frac{P_{X}}{M}\right) \sin (\Phi+\delta)\right]+\frac{\omega_{n}^{2} q}{\rho^{2}}\left[2 \cos 2 \Phi X Y-\sin 2 \Phi\left(X^{2}-Y^{2}\right)\right]
\end{aligned}
$$


substituting

$$
X=X_{M}-\frac{1}{\omega_{n}^{2}}\left(\frac{P_{X}}{M}\right), \dot{X}=\dot{X}_{M}, \quad \ddot{X}=\ddot{X}_{M}, \quad Y=Y_{M}-\frac{1}{\omega_{n}^{2}}\left(\frac{P_{Y}}{M}\right), \dot{Y}=\dot{Y}_{M}, \ddot{Y}=\ddot{Y}_{M}
$$

$\ddot{\Phi}+\omega_{t}^{2}(\Phi-\Theta)+2 \varsigma_{t} \omega_{t}(\dot{\Phi}-\dot{\Theta})=\frac{\varepsilon 2 \varsigma \omega_{n}}{\rho^{2}}\left[\cos (\Phi+\delta) \dot{Y}_{M}-\sin (\Phi+\delta) \dot{X}_{M}\right]$

$+\frac{\varepsilon \omega_{n}^{2}}{\rho^{2}}\left[\cos (\Phi+\delta) Y_{M}-\sin (\Phi+\delta) X_{M}\right]+\frac{\varepsilon \omega_{n}^{2} q}{\rho^{2}}\left[\cos (\Phi-\delta) Y_{M}-\sin (\Phi-\delta) X_{M}\right]$

$-\frac{\varepsilon}{\rho^{2}}\left[\left(\frac{P_{Y}}{M}\right) \cos (\Phi+\delta)-\left(\frac{P_{X}}{M}\right) \sin (\Phi+\delta)\right]-\frac{\varepsilon q}{\rho^{2}}\left[\left(\frac{P_{Y}}{M}\right) \cos (\Phi-\delta)-\left(\frac{P_{X}}{M}\right) \sin (\Phi-\delta)\right]$

$+\frac{\varepsilon}{\rho^{2}}\left[\left(\frac{P_{Y}}{M}\right) \cos (\Phi+\delta)-\left(\frac{P_{X}}{M}\right) \sin (\Phi+\delta)\right]$

$+\frac{\omega_{n}^{2} q}{\rho^{2}}\left[2 \cos 2 \Phi\left(X_{M}-\frac{1}{\omega_{n}^{2}}\left(\frac{P_{X}}{M}\right)\right)\left(Y_{M}-\frac{1}{\omega_{n}^{2}}\left(\frac{P_{Y}}{M}\right)\right)-\sin 2 \Phi\left(\left(X_{M}-\frac{1}{\omega_{n}^{2}}\left(\frac{P_{X}}{M}\right)\right)^{2}-\left(Y_{M}-\frac{1}{\omega_{n}^{2}}\left(\frac{P_{Y}}{M}\right)\right)^{2}\right)\right]$

$\ddot{\Phi}+\omega_{t}^{2}(\Phi-\Theta)+2 \varsigma_{t} \omega_{t}(\dot{\Phi}-\dot{\Theta})=\frac{\varepsilon 2 \varsigma \omega_{n}}{\rho^{2}}\left[\cos (\Phi+\delta) \dot{Y}_{M}-\sin (\Phi+\delta) \dot{X}_{M}\right]$

$+\frac{\varepsilon \omega_{n}^{2}}{\rho^{2}}\left[\cos (\Phi+\delta) Y_{M}-\sin (\Phi+\delta) X_{M}\right]+\frac{\varepsilon \omega_{n}^{2} q}{\rho^{2}}\left[\cos (\Phi-\delta) Y_{M}-\sin (\Phi-\delta) X_{M}\right]$

$-\frac{\varepsilon q}{\rho^{2}}\left[\left(\frac{P_{Y}}{M}\right) \cos (\Phi-\delta)-\left(\frac{P_{X}}{M}\right) \sin (\Phi-\delta)\right]$

$+\frac{\omega_{n}^{2} q}{\rho^{2}}\left[2 \cos 2 \Phi\left(X_{M}-\frac{1}{\omega_{n}^{2}}\left(\frac{P_{X}}{M}\right)\right)\left(Y_{M}-\frac{1}{\omega_{n}^{2}}\left(\frac{P_{Y}}{M}\right)\right)-\sin 2 \Phi\left(\left(X_{M}-\frac{1}{\omega_{n}^{2}}\left(\frac{P_{X}}{M}\right)\right)^{2}-\left(Y_{M}-\frac{1}{\omega_{n}^{2}}\left(\frac{P_{Y}}{M}\right)\right)^{2}\right)\right]$ 
aside

$$
\begin{aligned}
& 2 \cos 2 \Phi\left(X_{M}-\frac{1}{\omega_{n}^{2}}\left(\frac{P_{X}}{M}\right)\right)\left(Y_{M}-\frac{1}{\omega_{n}^{2}}\left(\frac{P_{Y}}{M}\right)\right)-\sin 2 \Phi\left(\left(X_{M}-\frac{1}{\omega_{n}^{2}}\left(\frac{P_{X}}{M}\right)\right)^{2}-\left(Y_{M}-\frac{1}{\omega_{n}^{2}}\left(\frac{P_{Y}}{M}\right)\right)^{2}\right) \\
& =2 \cos 2 \Phi\left(X_{M} Y_{M}-\frac{1}{\omega_{n}^{2}}\left(\frac{P_{X}}{M}\right) Y_{M}-\frac{1}{\omega_{n}^{2}}\left(\frac{P_{Y}}{M}\right) X_{M}+\frac{1}{\omega_{n}^{4}}\left(\frac{P_{Y}}{M}\right)\left(\frac{P_{X}}{M}\right)\right) \\
& -\sin 2 \Phi\left(X_{M}^{2}-\frac{2}{\omega_{n}^{2}}\left(\frac{P_{X}}{M}\right) X_{M}+\frac{1}{\omega_{n}^{4}}\left(\frac{P_{X}}{M}\right)^{2}\right)+\sin 2 \Phi\left(Y_{M}^{2}-\frac{2}{\omega_{n}^{2}}\left(\frac{P_{Y}}{M}\right) Y_{M}+\frac{1}{\omega_{n}^{4}}\left(\frac{P_{Y}}{M}\right)^{2}\right)
\end{aligned}
$$$$
2 \cos 2 \Phi\left(X_{M}-\frac{1}{\omega_{n}^{2}}\left(\frac{P_{X}}{M}\right)\right)\left(Y_{M}-\frac{1}{\omega_{n}^{2}}\left(\frac{P_{Y}}{M}\right)\right)-\sin 2 \Phi\left(\left(X_{M}-\frac{1}{\omega_{n}^{2}}\left(\frac{P_{X}}{M}\right)\right)^{2}-\left(Y_{M}-\frac{1}{\omega_{n}^{2}}\left(\frac{P_{Y}}{M}\right)\right)^{2}\right)
$$$$
=\frac{2}{\omega_{n}^{2}}\left[\cos 2 \Phi\left(-\left(\frac{P_{Y}}{M}\right) X_{M}-\left(\frac{P_{X}}{M}\right) Y_{M}\right)-\sin 2 \Phi\left(\left(\frac{P_{Y}}{M}\right) Y_{M}-\left(\frac{P_{X}}{M}\right) X_{M}\right)\right]
$$$$
+\frac{1}{\omega_{n}^{4}}\left[2 \cos 2 \Phi\left(\frac{P_{Y}}{M}\right)\left(\frac{P_{X}}{M}\right)-\sin 2 \Phi\left(\frac{P_{X}}{M}\right)^{2}+\sin 2 \Phi\left(\frac{P_{Y}}{M}\right)^{2}\right]
$$

$+2 \cos 2 \Phi X_{M} Y_{M}-\sin 2 \Phi X_{M}^{2}+\sin 2 \Phi Y_{M}^{2}$ 
combining

$$
\begin{aligned}
& \ddot{\Phi}+\omega_{t}^{2}(\Phi-\Theta)+2 \varsigma_{t} \omega_{t}(\dot{\Phi}-\dot{\Theta})=-\frac{\varepsilon q}{\rho^{2}}\left[\left(\frac{P_{Y}}{M}\right) \cos (\Phi-\delta)-\left(\frac{P_{X}}{M}\right) \sin (\Phi-\delta)\right] \\
& +\frac{q}{\omega_{n}^{2} \rho^{2}}\left[2 \cos 2 \Phi\left(\frac{P_{Y}}{M}\right)\left(\frac{P_{X}}{M}\right)-\sin 2 \Phi\left(\frac{P_{X}}{M}\right)^{2}+\sin 2 \Phi\left(\frac{P_{Y}}{M}\right)^{2}\right]+\Gamma
\end{aligned}
$$

where

$$
\begin{aligned}
& \Gamma=\frac{\varepsilon 2 \varsigma \omega_{n}}{\rho^{2}}\left[\cos (\Phi+\delta) \dot{Y}_{M}-\sin (\Phi+\delta) \dot{X}_{M}\right] \\
& +\frac{\varepsilon \omega_{n}^{2}}{\rho^{2}}\left[\cos (\Phi+\delta) Y_{M}-\sin (\Phi+\delta) X_{M}\right]+\frac{\varepsilon \omega_{n}^{2} q}{\rho^{2}}\left[\cos (\Phi-\delta) Y_{M}-\sin (\Phi-\delta) X_{M}\right] \\
& +\frac{2 q}{\rho^{2}}\left[\cos 2 \Phi\left(-\left(\frac{P_{Y}}{M}\right) X_{M}-\left(\frac{P_{X}}{M}\right) Y_{M}\right)-\sin 2 \Phi\left(\left(\frac{P_{Y}}{M}\right) Y_{M}-\left(\frac{P_{X}}{M}\right) X_{M}\right)\right] \\
& +\frac{\omega_{n}^{2} q}{\rho^{2}}\left[2 \cos 2 \Phi X_{M} Y_{M}-\sin 2 \Phi X_{M}^{2}+\sin 2 \Phi Y_{M}^{2}\right]
\end{aligned}
$$

substituting $\Phi=\Omega t+\varphi-\Phi_{0}, \dot{\Phi}=\Omega+\dot{\varphi}, \ddot{\Phi}=\ddot{\varphi}, \Theta=\Omega t+\theta-\Theta_{0}, \dot{\Theta}=\Omega+\dot{\theta}, \ddot{\Theta}=\ddot{\theta}$

$$
\begin{aligned}
& \ddot{\varphi}+\omega_{t}^{2}\left(\varphi-\theta-\Phi_{0}+\Theta_{0}\right)+2 \varsigma_{t} \omega_{t}(\dot{\varphi}-\dot{\theta})=-\frac{\varepsilon q}{\rho^{2}}\left[\left(\frac{P_{Y}}{M}\right) \cos \left(\Omega t+\varphi-\Phi_{0}-\delta\right)-\left(\frac{P_{X}}{M}\right) \sin \left(\Omega t+\varphi-\Phi_{0}-\delta\right)\right] \\
& +\frac{q}{\omega_{n}^{2} \rho^{2}}\left[2 \cos \left(2\left(\Omega t+\varphi-\Phi_{0}\right)\right)\left(\frac{P_{Y}}{M}\right)\left(\frac{P_{X}}{M}\right)-\sin \left(2\left(\Omega t+\varphi-\Phi_{0}\right)\right)\left(\frac{P_{X}}{M}\right)^{2}+\sin \left(2\left(\Omega t+\varphi-\Phi_{0}\right)\right)\left(\frac{P_{Y}}{M}\right)^{2}\right]+\Gamma
\end{aligned}
$$

where 


$$
\begin{aligned}
& \Gamma=\frac{\varepsilon 2 \varsigma \omega_{n}}{\rho^{2}}\left[\cos \left(\Omega t+\varphi-\Phi_{0}+\delta\right) \dot{Y}_{M}-\sin \left(\Omega t+\varphi-\Phi_{0}+\delta\right) \dot{X}_{M}\right] \\
& +\frac{\varepsilon \omega_{n}^{2}}{\rho^{2}}\left[\cos \left(\Omega t+\varphi-\Phi_{0}+\delta\right) Y_{M}-\sin \left(\Omega t+\varphi-\Phi_{0}+\delta\right) X_{M}\right] \\
& +\frac{\varepsilon \omega_{n}^{2} q}{\rho^{2}}\left[\cos \left(\Omega t+\varphi-\Phi_{0}-\delta\right) Y_{M}-\sin \left(\Omega t+\varphi-\Phi_{0}-\delta\right) X_{M}\right] \\
& +\frac{2 q}{\rho^{2}}\left[\cos \left(2\left(\Omega t+\varphi-\Phi_{0}\right)\right)\left(-\left(\frac{P_{Y}}{M}\right) X_{M}-\left(\frac{P_{X}}{M}\right) Y_{M}\right)-\sin \left(2\left(\Omega t+\varphi-\Phi_{0}\right)\right)\left(\left(\frac{P_{Y}}{M}\right) Y_{M}-\left(\frac{P_{X}}{M}\right) X_{M}\right)\right] \\
& +\frac{\omega_{n}^{2} q}{\rho^{2}}\left[2 \cos \left(2\left(\Omega t+\varphi-\Phi_{0}\right)\right) X_{M} Y_{M}-\sin \left(2\left(\Omega t+\varphi-\Phi_{0}\right)\right) X_{M}^{2}+\sin \left(2\left(\Omega t+\varphi-\Phi_{0}\right)\right) Y_{M}^{2}\right]
\end{aligned}
$$


let $\tau=\omega_{n} t, \frac{d(\square)}{d t}=\omega_{n} \frac{d(\square)}{d \tau}=\omega_{n}(\square)^{\prime} \frac{d^{2}(\square)}{d t^{2}}=\omega_{n}^{2} \frac{d^{2}(\square)}{d \tau^{2}}=\omega_{n}^{2}(\square)^{\prime \prime}$

$$
\begin{aligned}
& \varphi^{\prime \prime}+\left(\frac{\omega_{t}}{\omega_{n}}\right)^{2}\left(\varphi-\theta-\Phi_{0}+\Theta_{0}\right)+\frac{2 \varsigma_{t} \omega_{t}}{\omega_{n}}\left(\varphi^{\prime}-\theta^{\prime}\right)=-\frac{\varepsilon q}{\omega_{n}^{2} \rho^{2}}\left[\left(\frac{P_{Y}}{M}\right) \cos \left(\frac{\Omega}{\omega_{n}} \tau+\varphi-\Phi_{0}-\delta\right)-\left(\frac{P_{X}}{M}\right) \sin \left(\frac{\Omega}{\omega_{n}} \tau+\varphi-\Phi_{0}-\delta\right)\right] \\
& +\frac{q}{\omega_{n}^{4} \rho^{2}}\left[2 \cos \left(2\left(\frac{\Omega}{\omega_{n}} \tau+\varphi-\Phi_{0}\right)\right)\left(\frac{P_{Y}}{M}\right)\left(\frac{P_{X}}{M}\right)-\sin \left(2\left(\frac{\Omega}{\omega_{n}} \tau+\varphi-\Phi_{0}\right)\right)\left(\frac{P_{X}}{M}\right)^{2}+\sin \left(2\left(\frac{\Omega}{\omega_{n}} \tau+\varphi-\Phi_{0}\right)\right)\left(\frac{P_{Y}}{M}\right)^{2}\right]+\Gamma_{n} \\
& \Gamma_{n}=\frac{\varepsilon 2 \varsigma}{\rho^{2}}\left[\cos \left(\frac{\Omega}{\omega_{n}} \tau+\varphi-\Phi_{0}+\delta\right) Y_{M}^{\prime}-\sin \left(\frac{\Omega}{\omega_{n}} \tau+\varphi-\Phi_{0}+\delta\right) X_{M}^{\prime}\right] \\
& +\frac{\varepsilon}{\rho^{2}}\left[\cos \left(\frac{\Omega}{\omega_{n}} \tau+\varphi-\Phi_{0}+\delta\right) Y_{M}-\sin \left(\frac{\Omega}{\omega_{n}} \tau+\varphi-\Phi_{0}+\delta\right) X_{M}\right] \\
& +\frac{\varepsilon q}{\rho^{2}}\left[\cos \left(\frac{\Omega}{\omega_{n}} \tau+\varphi-\Phi_{0}-\delta\right) Y_{M}-\sin \left(\frac{\Omega}{\omega_{n}} \tau+\varphi-\Phi_{0}-\delta\right) X_{M}\right] \\
& +\frac{2 q}{\omega_{n}^{2} \rho^{2}}\left[\cos \left(2\left(\frac{\Omega}{\omega_{n}} \tau+\varphi-\Phi_{0}\right)\right)\left(-\left(\frac{P_{Y}}{M}\right) X_{M}-\left(\frac{P_{X}}{M}\right) Y_{M}\right)-\sin \left(2\left(\frac{\Omega}{\omega_{n}} \tau+\varphi-\Phi_{0}\right)\right)\left(\left(\frac{P_{Y}}{M}\right) Y_{M}-\left(\frac{P_{X}}{M}\right) X_{M}\right)\right] \\
& +\frac{q}{\rho^{2}}\left[2 \cos \left(2\left(\frac{\Omega}{\omega_{n}} \tau+\varphi-\Phi_{0}\right)\right) X_{M} Y_{M}-\sin \left(2\left(\frac{\Omega}{\omega_{n}} \tau+\varphi-\Phi_{0}\right)\right) X_{M}^{2}+\sin \left(2\left(\frac{\Omega}{\omega_{n}} \tau+\varphi-\Phi_{0}\right)\right) Y_{M}^{2}\right]
\end{aligned}
$$


Lagrange Equation for $\Theta$

$$
\begin{aligned}
& \frac{\partial}{\partial t}\left(\frac{\partial T}{\partial \dot{\Theta}}\right)-\frac{\partial T}{\partial \Theta}+\frac{\partial U}{\partial \Theta}+\frac{\partial D}{\partial \dot{\Theta}}=-T_{D R} \\
& I_{0} \ddot{\Theta}-k_{t}(\Phi-\Theta)-C_{t}(\dot{\Phi}-\dot{\Theta})=-T_{D R} \\
& \ddot{\Theta}-\frac{k_{t}}{I_{0}}(\Phi-\Theta)-\frac{C_{t}}{I_{0}}(\dot{\Phi}-\dot{\Theta})=-\frac{T_{D R}}{I_{0}} \\
& \ddot{\Theta}-\frac{k_{t}}{I_{0}}(\Phi-\Theta)-\frac{C_{t}}{I_{0}}(\dot{\Phi}-\dot{\Theta})=-\frac{k_{c}}{I_{0}} \theta-\frac{C_{c}}{I_{0}} \dot{\theta}
\end{aligned}
$$

substituting $\Phi=\Omega t+\varphi-\Phi_{0}, \dot{\Phi}=\Omega+\dot{\varphi}, \ddot{\Phi}=\ddot{\varphi}, \Theta=\Omega t+\theta-\Theta_{0}, \dot{\Theta}=\Omega+\dot{\theta}, \ddot{\Theta}=\ddot{\theta}$

$$
\begin{aligned}
& I_{0} \ddot{\theta}-k_{t}\left(\varphi-\Phi_{0}-\theta+\Theta_{0}\right)-C_{t}(\dot{\varphi}-\dot{\theta})=-k_{c} \theta-C_{c} \dot{\theta} \\
& \ddot{\theta}+\frac{C_{c}+C_{t}}{I_{0}} \dot{\theta}+\frac{k_{c}+k_{t}}{I_{0}} \theta=\frac{k_{t}}{I_{0}}\left(\varphi+\Theta_{0}-\Phi_{0}\right)+\frac{C_{t}}{I_{0}} \dot{\varphi}
\end{aligned}
$$

$\ddot{\theta}+\left(1+C_{R}\right) \frac{C_{t}}{I} \dot{\theta}+\left(1+K_{R}\right) \frac{k_{t}}{I} \theta=\frac{k_{t}}{I}\left(\varphi+\Theta_{0}-\Phi_{0}\right)+\frac{C_{t}}{I} \dot{\varphi}$

$\ddot{\theta}+\left(1+C_{R}\right) 2 \varsigma_{t} \omega_{t} \dot{\theta}+\left(1+K_{R}\right) \omega_{t}^{2} \theta=\omega_{t}^{2}\left(\varphi+\Theta_{0}-\Phi_{0}\right)+2 \varsigma_{t} \omega_{t} \dot{\varphi}$

let $\tau=\omega_{n} t, \frac{d(\square)}{d t}=\omega_{n} \frac{d(\square)}{d \tau}=\omega_{n}(\square)^{\prime}, \frac{d^{2}(\square)}{d t^{2}}=\omega_{n}^{2} \frac{d^{2}(\square)}{d \tau^{2}}=\omega_{n}^{2}(\square)^{\prime \prime}$

$$
\theta^{\prime \prime}+\left(1+C_{R}\right) 2 \varsigma_{t} \frac{\omega_{t}}{\omega_{n}} \theta^{\prime}+\left(1+K_{R}\right)\left(\frac{\omega_{t}}{\omega_{n}}\right)^{2} \theta=\left(\frac{\omega_{t}}{\omega_{n}}\right)^{2} \varphi+2 \varsigma_{t} \frac{\omega_{t}}{\omega_{n}} \varphi^{\prime}
$$


Special Case, Torsional Vibration Only

Assume $X_{M}=0, Y_{M}=0, \theta=0$

Let $P_{X}=0, \Phi_{0}=0, \Theta_{0}=0, \delta=0$

The torsional equation of motion becomes

$\varphi^{\prime \prime}+\left(\frac{\omega_{t}}{\omega_{n}}\right)^{2} \varphi+\frac{2 \varsigma_{t} \omega_{t}}{\omega_{n}} \varphi^{\prime}=-\frac{\varepsilon q}{\omega_{n}^{2} \rho^{2}}\left(\frac{P_{Y}}{M}\right) \cos \left(\frac{\Omega}{\omega_{n}} \tau+\varphi\right)+\frac{q}{\omega_{n}^{4} \rho^{2}} \sin \left(2\left(\frac{\Omega}{\omega_{n}} \tau+\varphi\right)\right)\left(\frac{P_{Y}}{M}\right)^{2}$

Let the states $u_{1}=\varphi$ and $u_{2}=\varphi^{\prime}$

$u_{1}^{\prime}=u_{2}$

$u_{2}^{\prime}=-\left(\frac{\omega_{t}}{\omega_{n}}\right)^{2} u_{1}-\frac{2 \varsigma_{t} \omega_{t}}{\omega_{n}} u_{2}-\frac{\varepsilon q}{\omega_{n}^{2} \rho^{2}}\left(\frac{P_{Y}}{M}\right) \cos \left(\frac{\Omega}{\omega_{n}} \tau+u_{1}\right)+\frac{q}{\omega_{n}^{4} \rho^{2}} \sin \left(2\left(\frac{\Omega}{\omega_{n}} \tau+u_{1}\right)\right)\left(\frac{P_{Y}}{M}\right)^{2}$ 
Special Case, Lateral Vibration Only

Assume $\varphi=0, \theta=0$

Let $P_{X}=0, \Phi_{0}=0, \Theta_{0}=0, \delta=0$

$X_{M}^{\prime \prime}+2 \varsigma X_{M}^{\prime}+\left(1-q \cos \left(2 \frac{\Omega}{\omega_{n}} \tau\right)\right) X_{M}-q \sin \left(2 \frac{\Omega}{\omega_{n}} \tau\right) Y_{M}=\frac{\varepsilon}{\omega_{n}^{2}} \Omega^{2} \cos \left(\frac{\Omega}{\omega_{n}} \tau\right)-\left(\frac{P_{Y}}{M}\right) \frac{q}{\omega_{n}^{2}} \sin \left(2 \frac{\Omega}{\omega_{n}} \tau\right)$

$Y_{M}^{\prime \prime}+2 \varsigma Y_{M}^{\prime}+\left(1+q \cos \left(2 \frac{\Omega}{\omega_{n}} \tau\right)\right) Y_{M}-q \sin \left(2 \frac{\Omega}{\omega_{n}} \tau\right) X_{M}=\frac{\varepsilon}{\omega_{n}^{2}} \Omega^{2} \sin \left(\frac{\Omega}{\omega_{n}} \tau\right)+\left(\frac{P_{Y}}{M}\right) \frac{q}{\omega_{n}^{2}} \cos \left(2 \frac{\Omega}{\omega_{n}} \tau\right)$

Let the states $u_{3}=X_{M}, u_{4}=X_{M}^{\prime}, u_{5}=Y_{M}, u_{6}=Y_{M}^{\prime}$

$u_{3}^{\prime}=u_{4}$

$u_{4}^{\prime}=-2 \varsigma u_{4}-\left(1-q \cos \left(2 \frac{\Omega}{\omega_{n}} \tau\right)\right) u_{3}+q \sin \left(2 \frac{\Omega}{\omega_{n}} \tau\right) u_{5}+\frac{\varepsilon}{\omega_{n}^{2}} \Omega^{2} \cos \left(\frac{\Omega}{\omega_{n}} \tau\right)-\left(\frac{P_{Y}}{M}\right) \frac{q}{\omega_{n}^{2}} \sin \left(2 \frac{\Omega}{\omega_{n}} \tau\right)$

$u_{5}^{\prime}=u_{6}$

$u_{6}^{\prime}=-2 \varsigma u_{6}-\left(1+q \cos \left(2 \frac{\Omega}{\omega_{n}} \tau\right)\right) u_{5}+q \sin \left(2 \frac{\Omega}{\omega_{n}} \tau\right) u_{3}+\frac{\varepsilon}{\omega_{n}^{2}} \Omega^{2} \sin \left(\frac{\Omega}{\omega_{n}} \tau\right)+\left(\frac{P_{Y}}{M}\right) \frac{q}{\omega_{n}^{2}} \cos \left(2 \frac{\Omega}{\omega_{n}} \tau\right)$ 
General Vibration

Let $P_{X}=0, \Phi_{0}=0, \Theta_{0}=0$

$$
\begin{aligned}
& \varphi^{\prime \prime}=\left(\frac{\omega_{t}}{\omega_{n}}\right)^{2}(\theta-\varphi)+\frac{2 \varsigma_{t} \omega_{t}}{\omega_{n}}\left(\theta^{\prime}-\varphi^{\prime}\right)-\frac{\varepsilon q}{\omega_{n}^{2} \rho^{2}}\left(\frac{P_{Y}}{M}\right) \cos \left(\frac{\Omega}{\omega_{n}} \tau+\varphi\right) \\
& +\frac{q}{\omega_{n}^{4} \rho^{2}} \sin \left(2\left(\frac{\Omega}{\omega_{n}} \tau+\varphi\right)\right)\left(\frac{P_{Y}}{M}\right)^{2}+\Gamma_{n} \\
& \Gamma_{n}=\frac{\varepsilon 2 \varsigma}{\rho^{2}}\left[\cos \left(\frac{\Omega}{\omega_{n}} \tau+\varphi\right) Y_{M}^{\prime}-\sin \left(\frac{\Omega}{\omega_{n}} \tau+\varphi\right) X_{M}^{\prime}\right] \\
& +\frac{\varepsilon}{\rho^{2}}\left[\cos \left(\frac{\Omega}{\omega_{n}} \tau+\varphi\right) Y_{M}-\sin \left(\frac{\Omega}{\omega_{n}} \tau+\varphi\right) X_{M}\right] \\
& +\frac{\varepsilon q}{\rho^{2}}\left[\cos \left(\frac{\Omega}{\omega_{n}} \tau+\varphi\right) Y_{M}-\sin \left(\frac{\Omega}{\omega_{n}} \tau+\varphi\right) X_{M}\right] \\
& +\frac{2 q}{\omega_{n}^{2} \rho^{2}}\left(\frac{P_{Y}}{M}\right)\left[-\cos \left(2\left(\frac{\Omega}{\omega_{n}} \tau+\varphi\right)\right) X_{M}-\sin \left(2\left(\frac{\Omega}{\omega_{n}} \tau+\varphi\right)\right) Y_{M}\right] \\
& +\frac{q}{\rho^{2}}\left[2 \cos \left(2\left(\frac{\Omega}{\omega_{n}} \tau+\varphi\right)\right) X_{M} Y_{M}-\sin \left(2\left(\frac{\Omega}{\omega_{n}} \tau+\varphi\right)\right) X_{M}^{2}+\sin \left(2\left(\frac{\Omega}{\omega_{n}} \tau+\varphi\right)\right) Y_{M}^{2}\right] \\
& \theta^{\prime \prime}=-\left(1+C_{R}\right) 2 \varsigma_{t} \frac{\omega_{t}}{\omega_{n}} \theta^{\prime}-\left(1+K_{R}\right)\left(\frac{\omega_{t}}{\omega_{n}}\right)^{2} \theta+\left(\frac{\omega_{t}}{\omega_{n}}\right)^{2} \varphi+2 \varsigma_{t} \frac{\omega_{t}}{\omega_{n}} \varphi^{\prime} \\
& X_{M}^{\prime \prime}=-2 \varsigma X_{M}^{\prime}-\left(1-q \cos \left(2\left(\frac{\Omega}{\omega_{n}} \tau+\varphi\right)\right)\right) X_{M}+q \sin \left(2\left(\frac{\Omega}{\omega_{n}} \tau+\varphi\right)\right) Y_{M}+\varepsilon \varphi^{\prime \prime} \sin \left(\frac{\Omega}{\omega_{n}} \tau+\varphi\right) \\
& +\frac{\varepsilon}{\omega_{n}^{2}}\left(\Omega+\omega_{n} \varphi^{\prime}\right)^{2} \cos \left(\frac{\Omega}{\omega_{n}} \tau+\varphi\right)-\left(\frac{P_{Y}}{M}\right) \frac{q}{\omega_{n}^{2}} \sin \left(2\left(\frac{\Omega}{\omega_{n}} \tau+\varphi\right)\right)
\end{aligned}
$$




$$
\begin{aligned}
& Y_{M}^{\prime \prime}=-2 \varsigma Y_{M}^{\prime}-\left(1+q \cos \left(2\left(\frac{\Omega}{\omega_{n}} \tau+\varphi\right)\right)\right) Y_{M}+q \sin \left(2\left(\frac{\Omega}{\omega_{n}} \tau+\varphi\right)\right) X_{M}-\varepsilon \varphi^{\prime \prime} \cos \left(\frac{\Omega}{\omega_{n}} \tau+\varphi\right) \\
& +\frac{\varepsilon}{\omega_{n}^{2}}\left(\Omega+\omega_{n} \varphi^{\prime}\right)^{2} \sin \left(\frac{\Omega}{\omega_{n}} \tau+\varphi\right)+\left(\frac{P_{Y}}{M}\right) \frac{q}{\omega_{n}^{2}} \cos \left(2\left(\frac{\Omega}{\omega_{n}} \tau+\varphi-\Phi_{0}\right)\right) \\
& \text { Let the states } u_{1}=\varphi, u_{2}=\varphi^{\prime}, u_{3}=X_{M}, u_{4}=X_{M}^{\prime}, u_{5}=Y_{M}, u_{6}=Y_{M}^{\prime}, u_{7}=\theta \text {, and } u_{8}=\theta^{\prime} \\
& u_{1}^{\prime}=u_{2} \\
& u_{2}^{\prime}=\left(\frac{\omega_{t}}{\omega_{n}}\right)^{2}\left(u_{7}-u_{1}\right)+\frac{2 \varsigma_{t} \omega_{t}}{\omega_{n}}\left(u_{8}-u_{2}\right)-\frac{\varepsilon q}{\omega_{n}^{2} \rho^{2}}\left(\frac{P_{Y}}{M}\right) \cos \left(\frac{\Omega}{\omega_{n}} \tau+u_{1}\right) \\
& +\frac{q}{\omega_{n}^{4} \rho^{2}} \sin \left(2\left(\frac{\Omega}{\omega_{n}} \tau+u_{1}\right)\right)\left(\frac{P_{Y}}{M}\right)^{2}+\Gamma_{n} \\
& \Gamma_{n}=\frac{\varepsilon 2 \varsigma}{\rho^{2}}\left[\cos \left(\frac{\Omega}{\omega_{n}} \tau+u_{1}\right) u_{6}-\sin \left(\frac{\Omega}{\omega_{n}} \tau+u_{1}\right) u_{4}\right] \\
& +\frac{\varepsilon}{\rho^{2}}\left[\cos \left(\frac{\Omega}{\omega_{n}} \tau+u_{1}\right) u_{5}-\sin \left(\frac{\Omega}{\omega_{n}} \tau+u_{1}\right) u_{3}\right] \\
& +\frac{\varepsilon q}{\rho^{2}}\left[\cos \left(\frac{\Omega}{\omega_{n}} \tau+u_{1}\right) u_{5}-\sin \left(\frac{\Omega}{\omega_{n}} \tau+u_{1}\right) u_{3}\right] \\
& +\frac{2 q}{\omega_{n}^{2} \rho^{2}}\left(\frac{P_{Y}}{M}\right)\left[-\cos \left(2\left(\frac{\Omega}{\omega_{n}} \tau+u_{1}\right)\right) u_{3}-\sin \left(2\left(\frac{\Omega}{\omega_{n}} \tau+u_{1}\right)\right) u_{5}\right] \\
& +\frac{q}{\rho^{2}}\left[2 \cos \left(2\left(\frac{\Omega}{\omega_{n}} \tau+u_{1}\right)\right) u_{3} u_{5}-\sin \left(2\left(\frac{\Omega}{\omega_{n}} \tau+u_{1}\right)\right) u_{3}^{2}+\sin \left(2\left(\frac{\Omega}{\omega_{n}} \tau+u_{1}\right)\right) u_{5}^{2}\right] \\
& u_{8}^{\prime}=-\left(1+C_{R}\right) 2 \varsigma_{t} \frac{\omega_{t}}{\omega_{n}} u_{8}-\left(1+K_{R}\right)\left(\frac{\omega_{t}}{\omega_{n}}\right)^{2} u_{7}+\left(\frac{\omega_{t}}{\omega_{n}}\right)^{2} u_{1}+2 \varsigma_{t} \frac{\omega_{t}}{\omega_{n}} u_{2} \\
& u_{4}^{\prime}=-2 \varsigma u_{4}-\left(1-q \cos \left(2\left(\frac{\Omega}{\omega_{n}} \tau+u_{1}\right)\right)\right) u_{3}+q \sin \left(2\left(\frac{\Omega}{\omega_{n}} \tau+u_{1}\right)\right) u_{5}+\varepsilon u_{2}^{\prime} \sin \left(\frac{\Omega}{\omega_{n}} \tau+u_{1}\right) \\
& +\frac{\varepsilon}{\omega_{n}^{2}}\left(\Omega+\omega_{n} u_{2}\right)^{2} \cos \left(\frac{\Omega}{\omega_{n}} \tau+u_{1}\right)-\left(\frac{P_{Y}}{M}\right) \frac{q}{\omega_{n}^{2}} \sin \left(2\left(\frac{\Omega}{\omega_{n}} \tau+u_{1}\right)\right)
\end{aligned}
$$




$$
\begin{aligned}
& Y_{M}^{\prime \prime}=-2 \varsigma Y_{M}^{\prime}-\left(1+q \cos \left(2\left(\frac{\Omega}{\omega_{n}} \tau+\varphi\right)\right)\right) Y_{M}+q \sin \left(2\left(\frac{\Omega}{\omega_{n}} \tau+\varphi\right)\right) X_{M}-\varepsilon \varphi^{\prime \prime} \cos \left(\frac{\Omega}{\omega_{n}} \tau+\varphi\right) \\
& +\frac{\varepsilon}{\omega_{n}^{2}}\left(\Omega+\omega_{n} \varphi^{\prime}\right)^{2} \sin \left(\frac{\Omega}{\omega_{n}} \tau+\varphi\right)+\left(\frac{P_{Y}}{M}\right) \frac{q}{\omega_{n}^{2}} \cos \left(2\left(\frac{\Omega}{\omega_{n}} \tau+\varphi-\Phi_{0}\right)\right)
\end{aligned}
$$




\section{Appendix C Matlab Models}

\section{C.1 Special Case: Lateral Vibration Matlab Model}

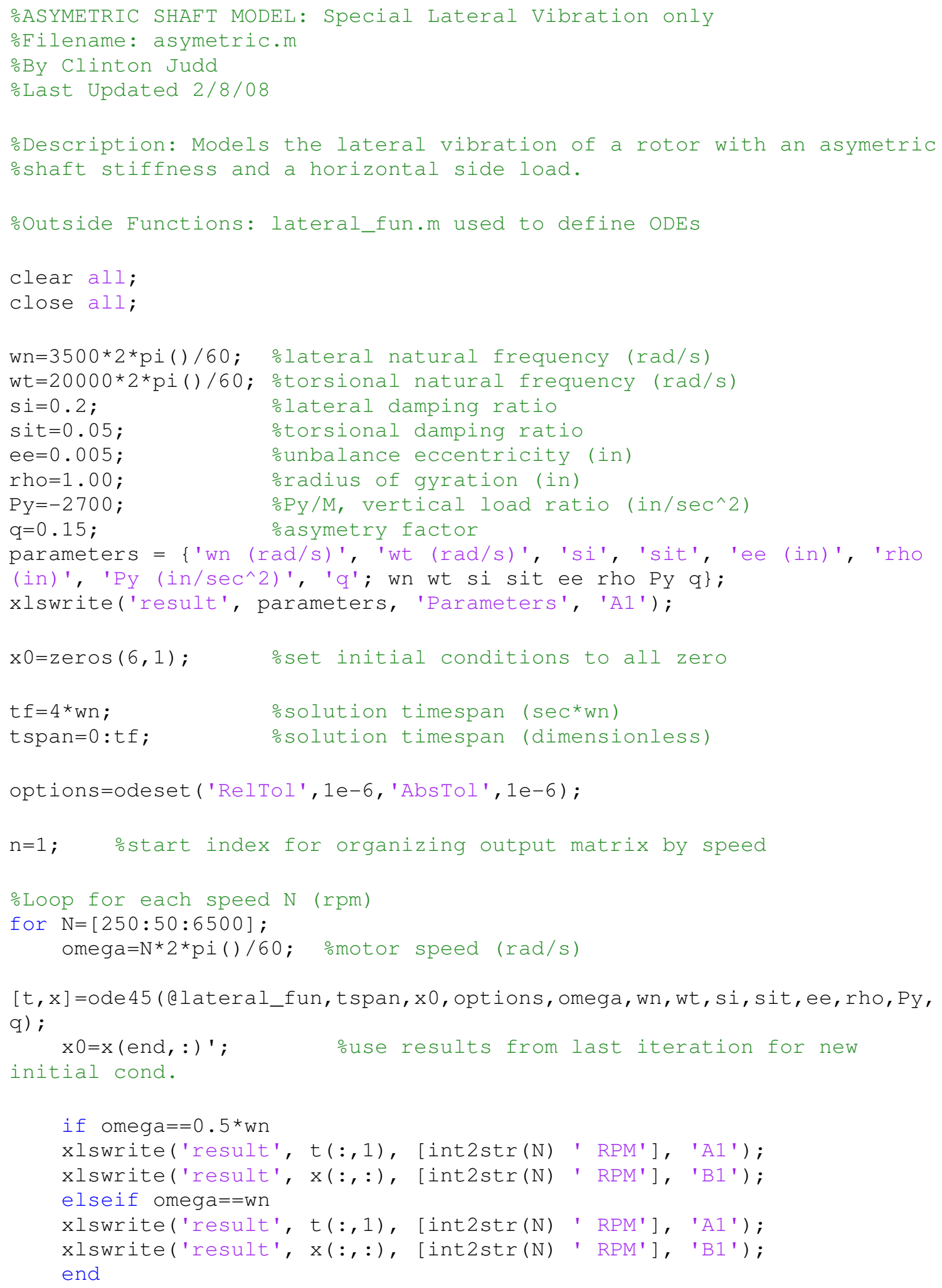




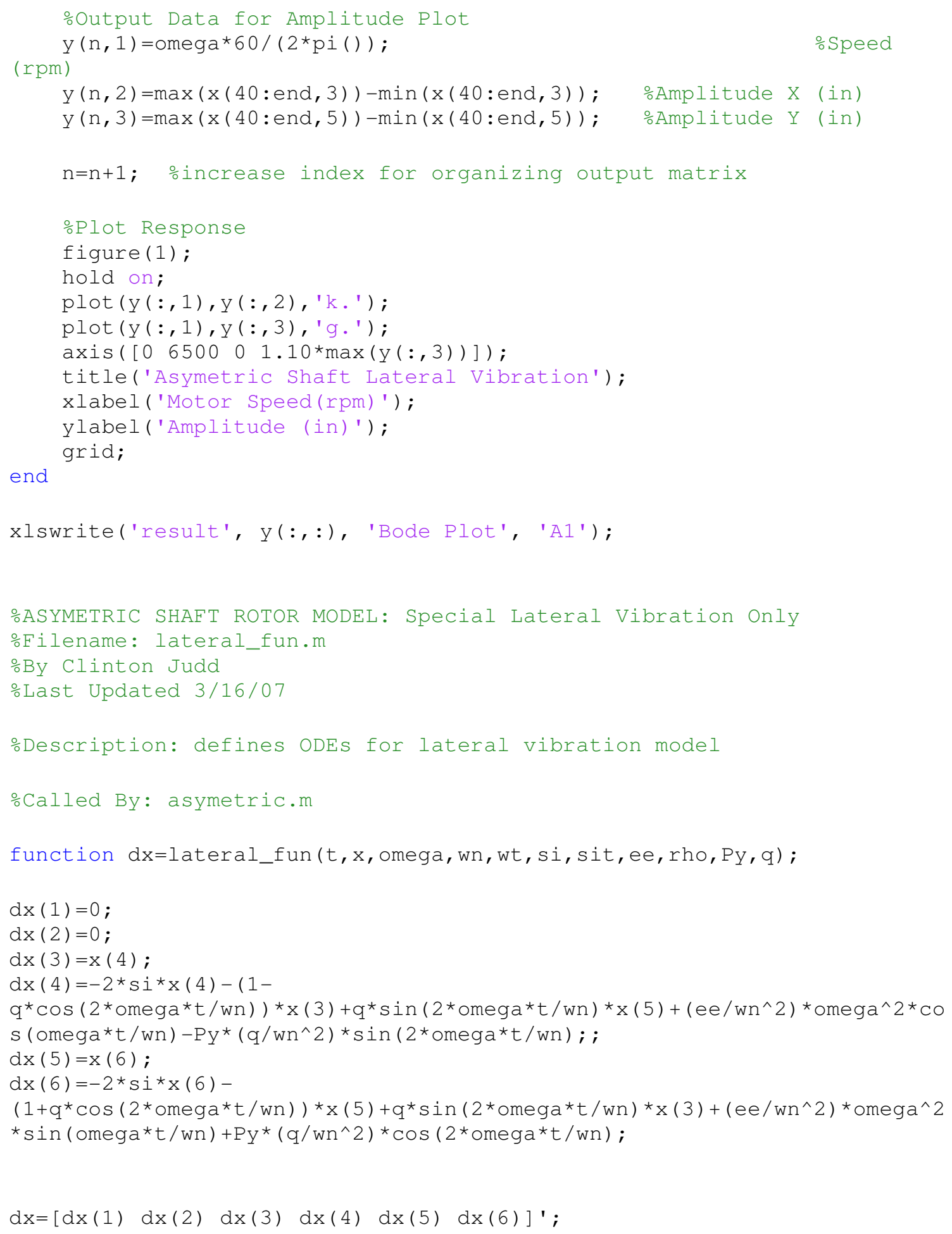




\section{C.2 Special Case: Torsional Vibration Matlab Model}

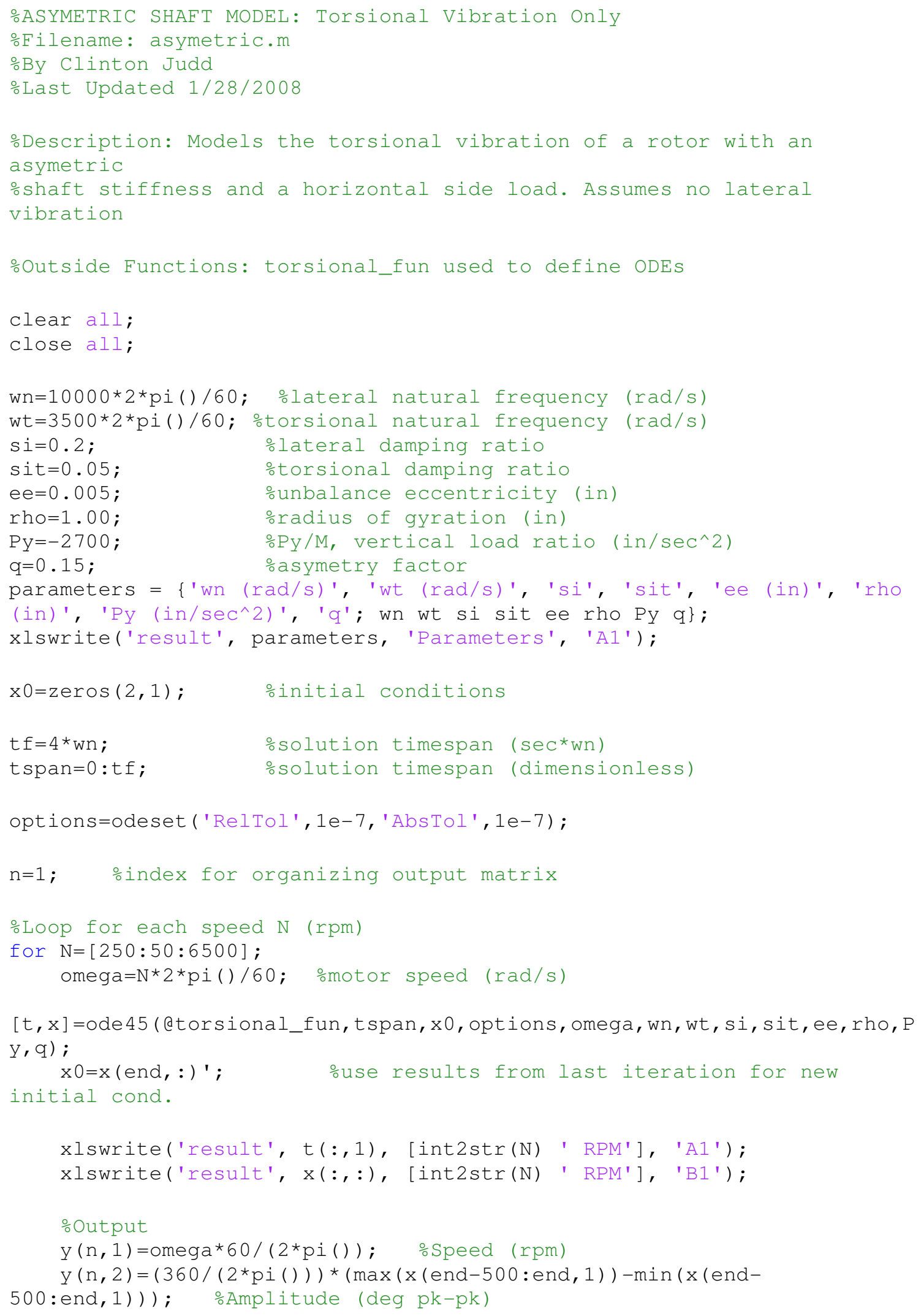




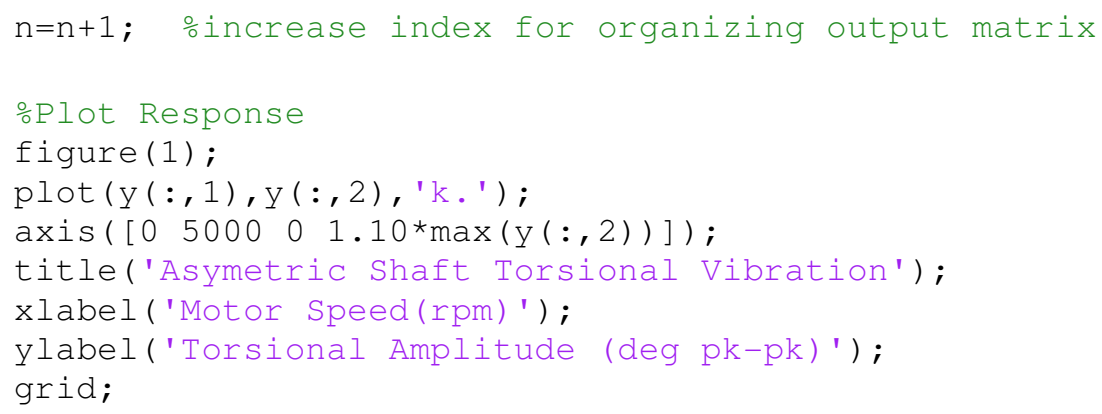

end

xlswrite('result', y(:,:), 'Bode Plot', 'Al');

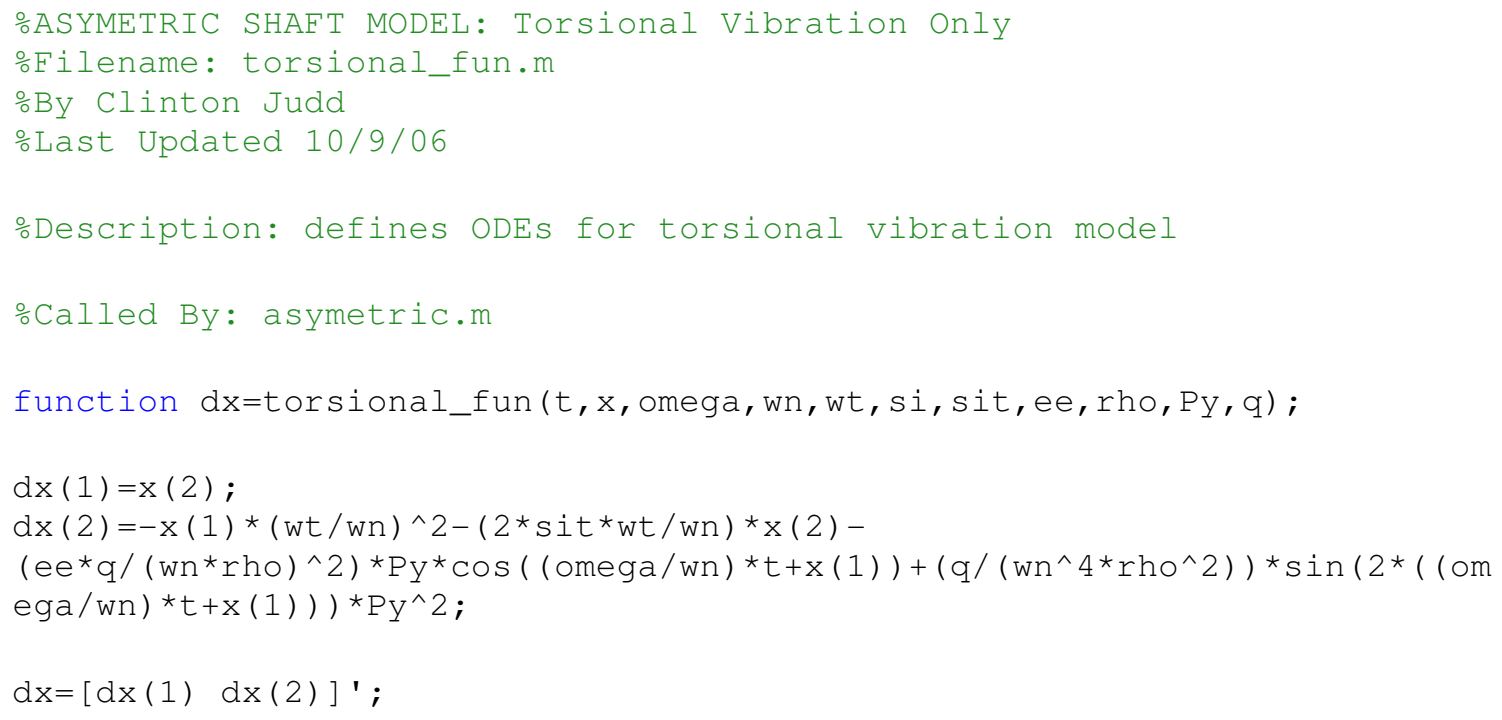




\section{C.3 Special Case: Torsional Vibration Matlab Model For Parametric Study Of $\frac{P_{Y}}{k} / \varepsilon$}

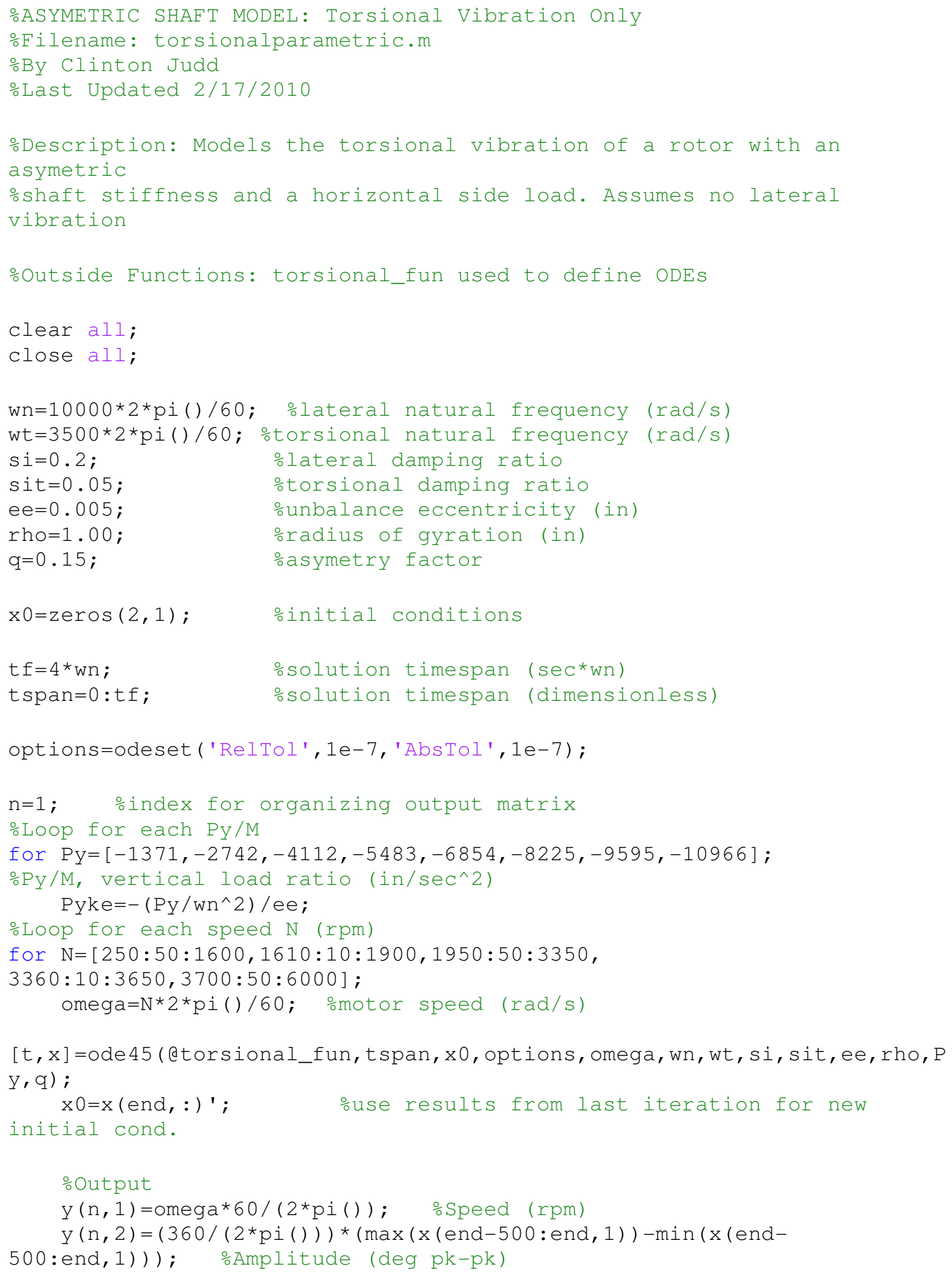




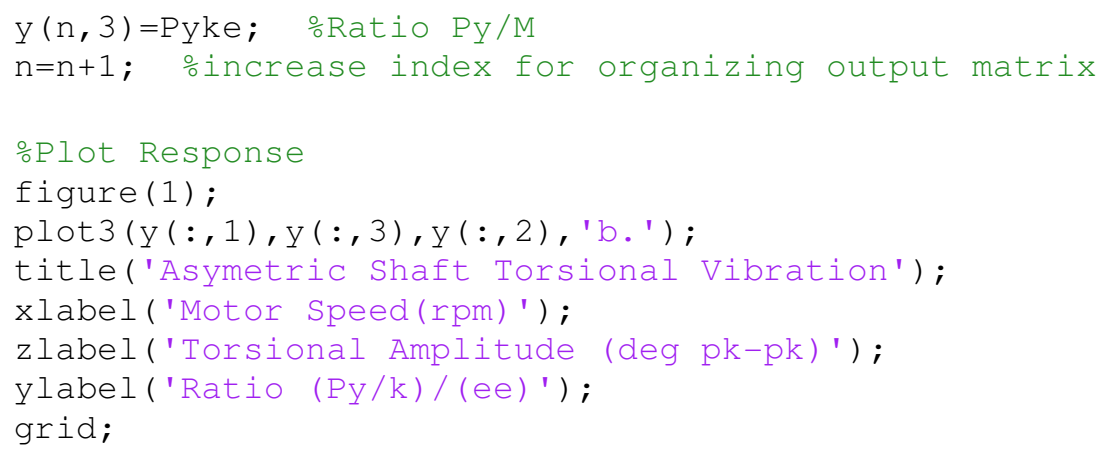

end

end

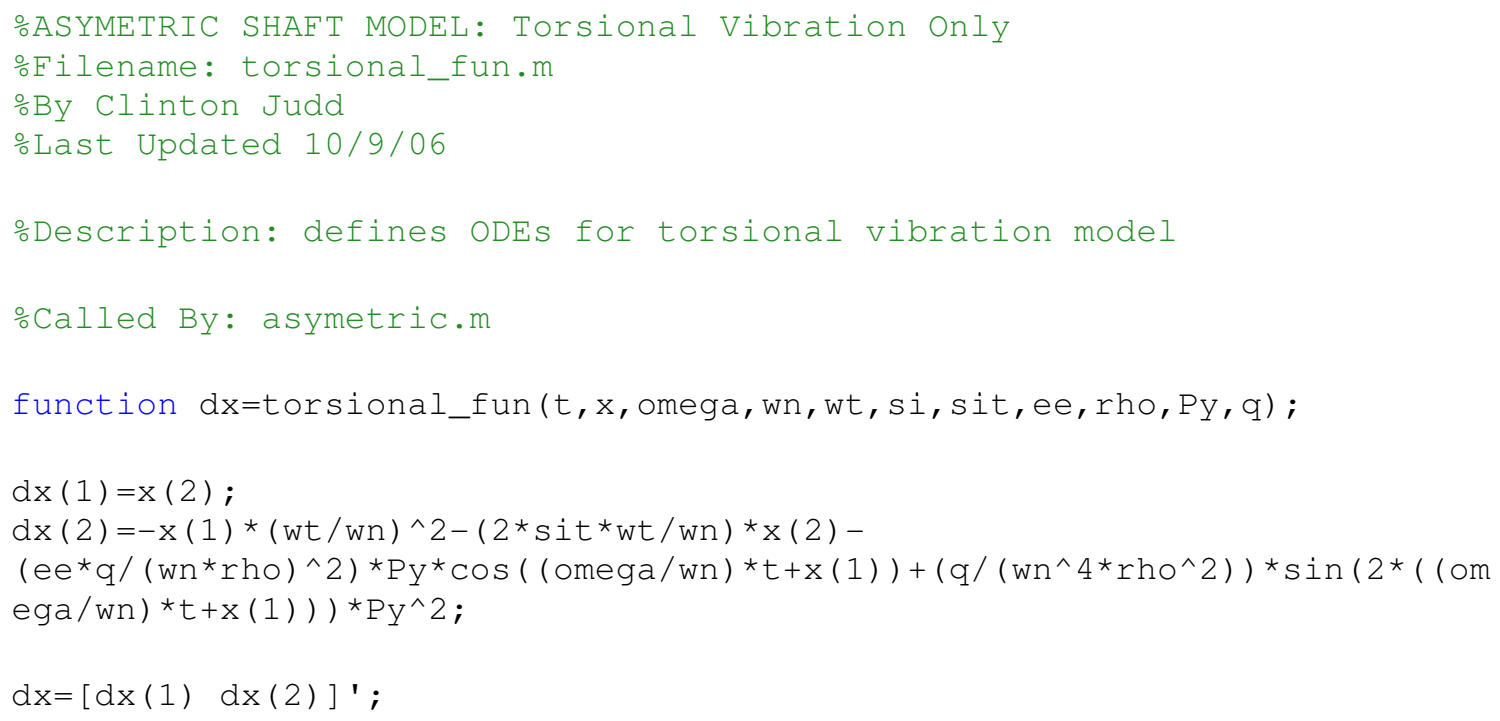




\section{C.4 General Vibration Model}

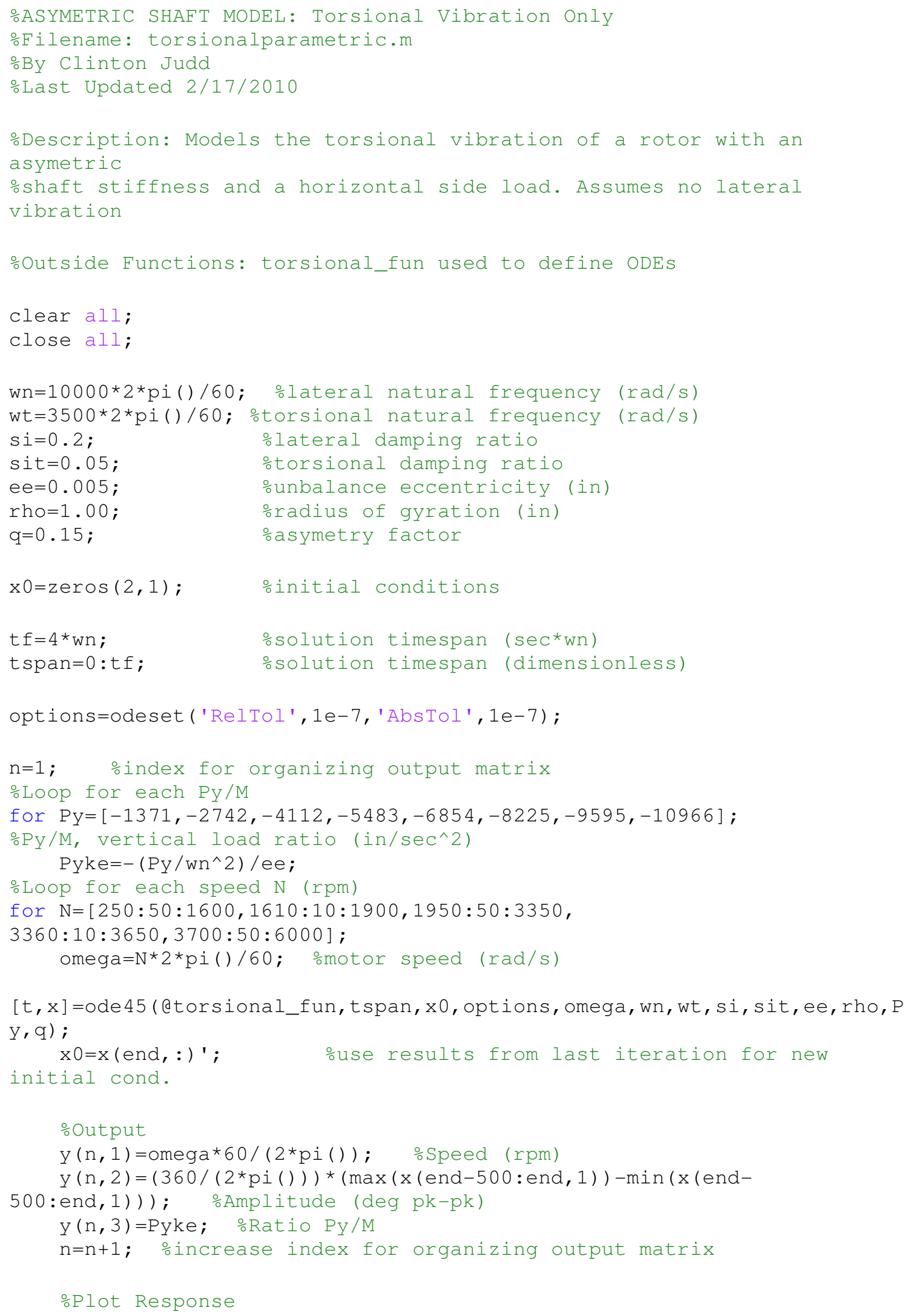


figure (1);

plot3 $(y(:, 1), y(:, 3), y(:, 2), ' b . ')$;

title('Asymetric Shaft Torsional Vibration');

xlabel ('Motor Speed(rpm)');

zlabel('Torsional Amplitude (deg pk-pk)');

ylabel ('Ratio (Py/k)/(ee)') ;

grid;

end

end

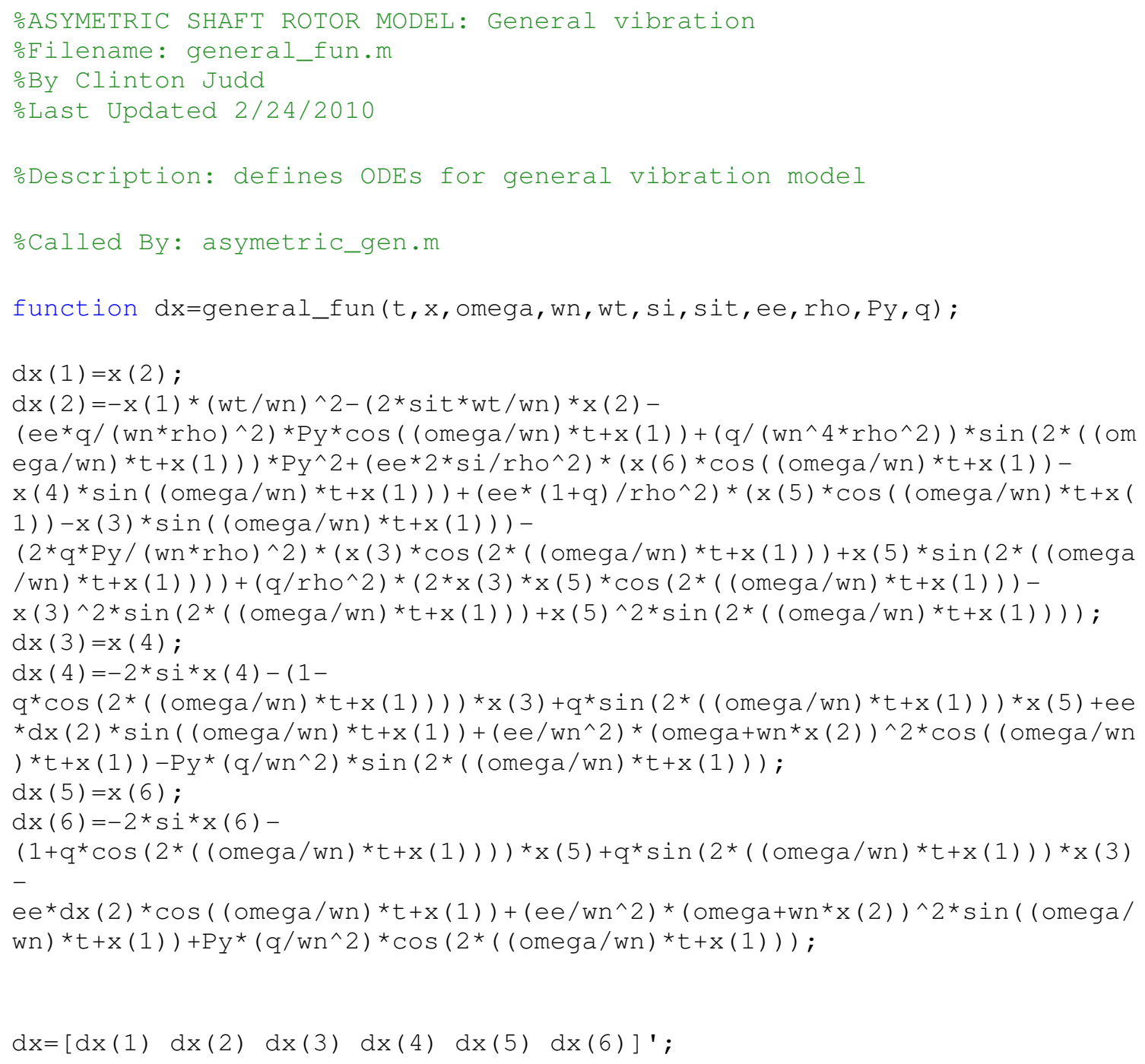




\section{C.5 Vibration Model for Increasing Shaft Asymmetry}

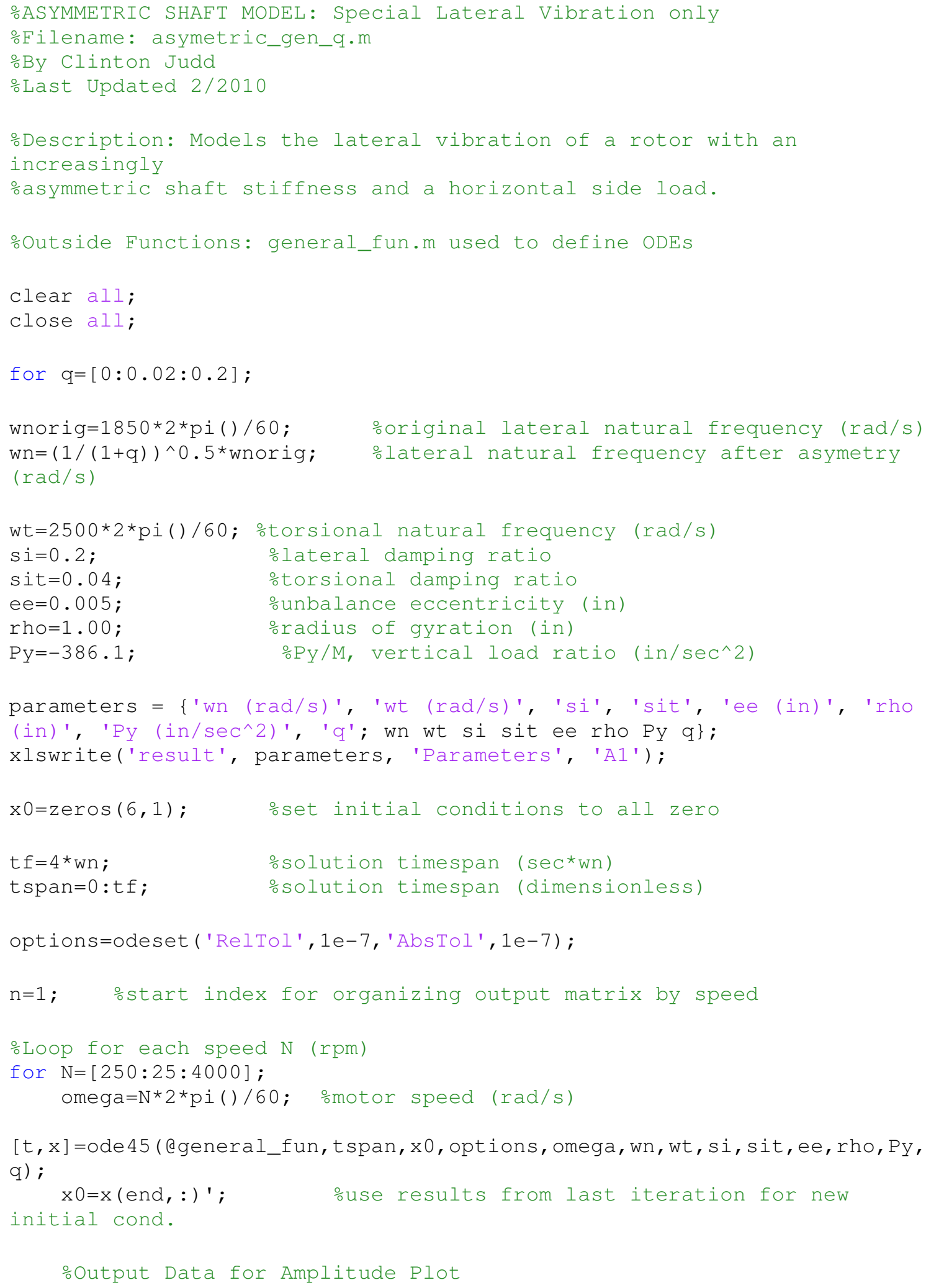




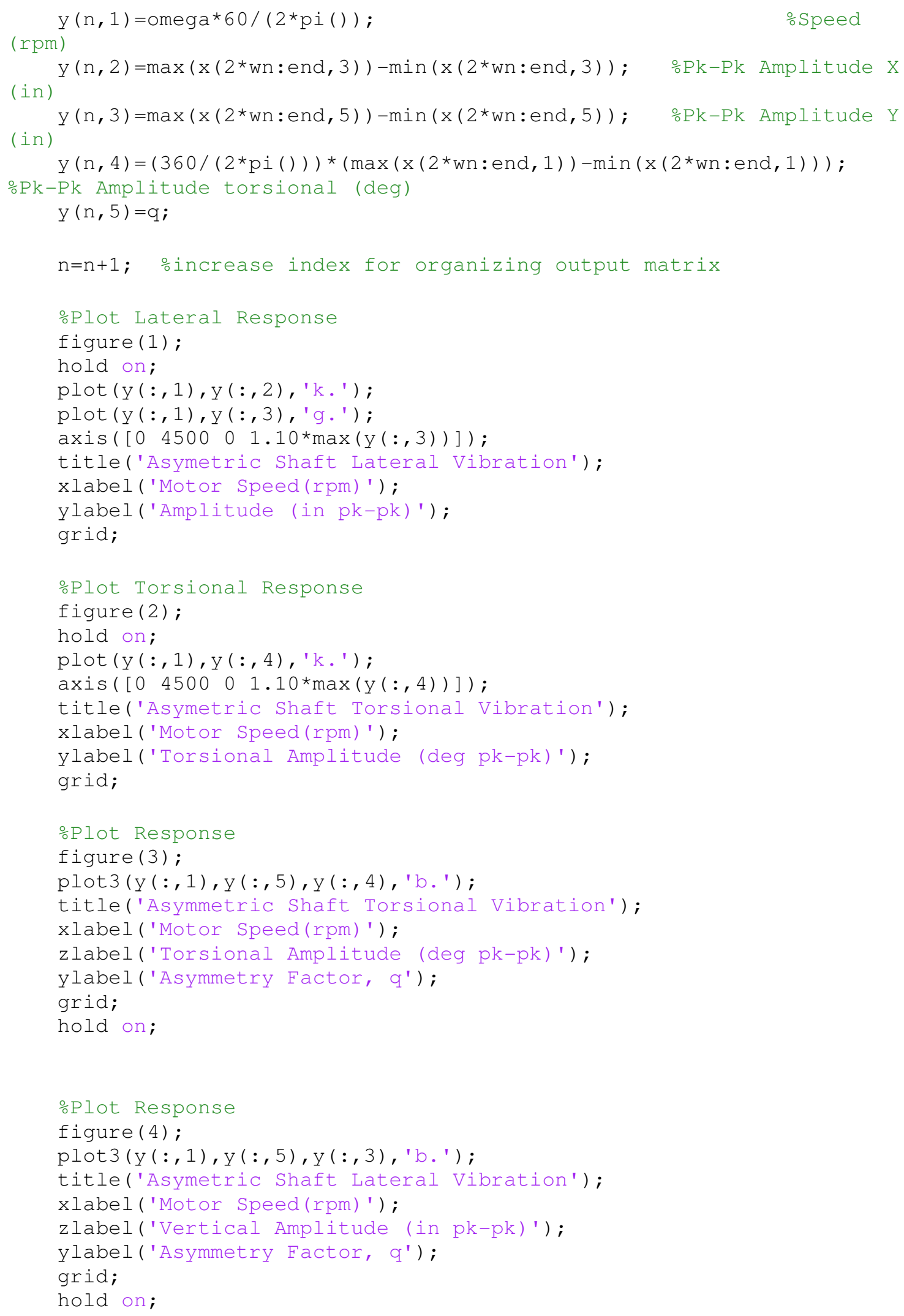


xlswrite('result', $y(:,:), \quad$ ['Bode Plot $q=$ ' int2str(q)], 'Al'); end

end 


\section{C.6 Vibration Model for Varying Ratios of $\omega_{t} / \omega_{n}$}

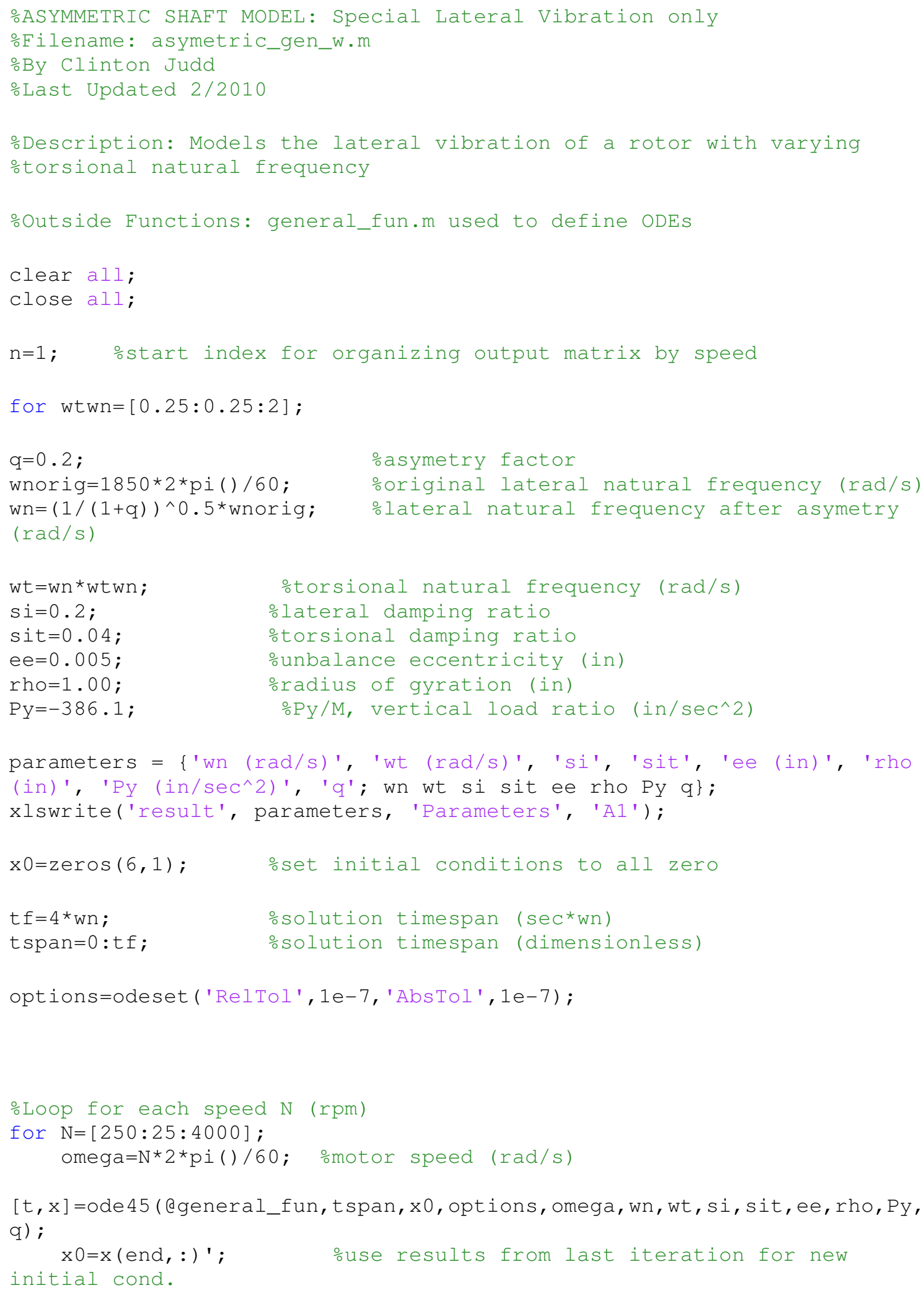




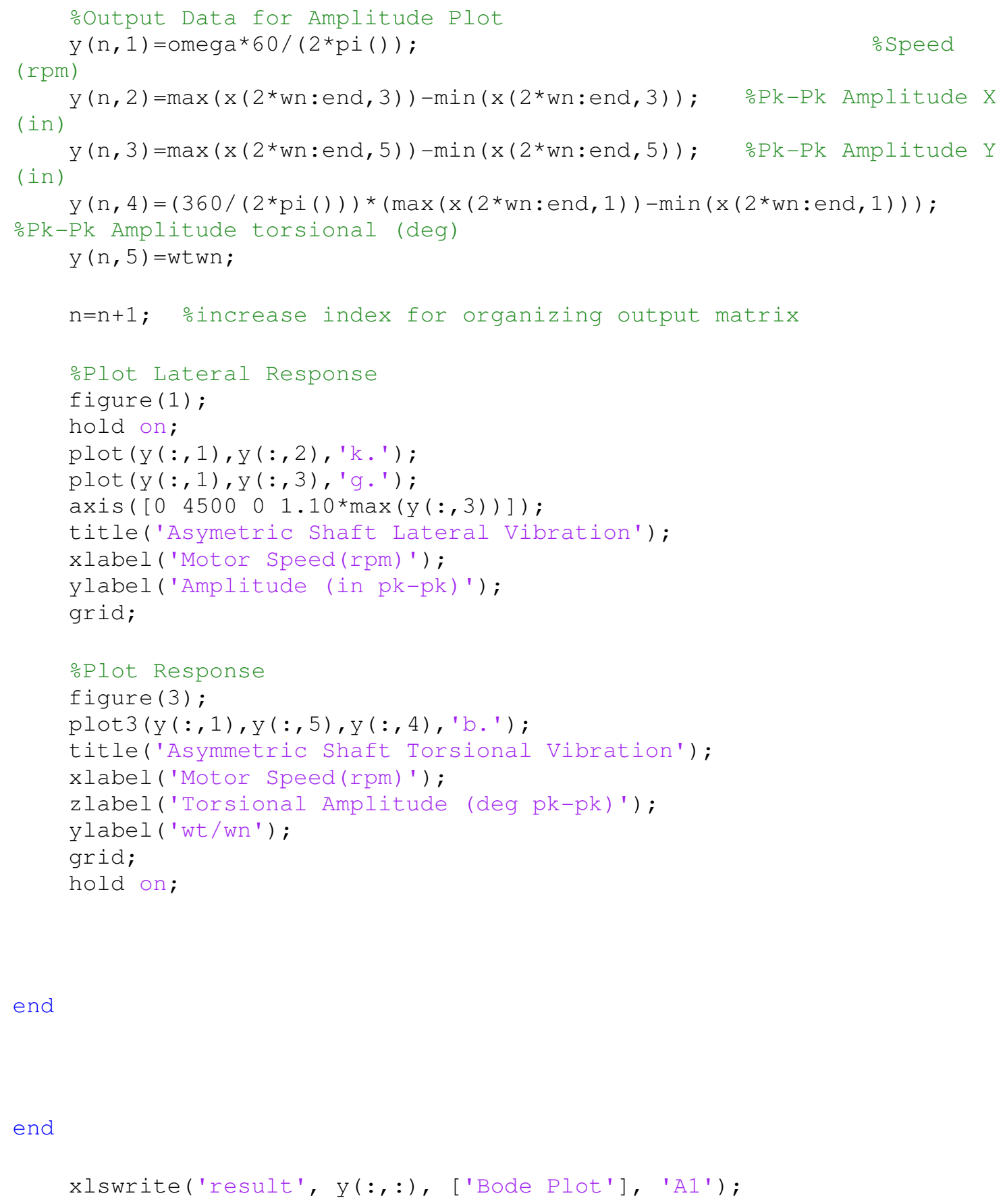

end

end

xlswrite('result', y(:,:), ['Bode Plot'], 'Al'); 


\section{C.7 Vibration Model for Increasing Side Load}

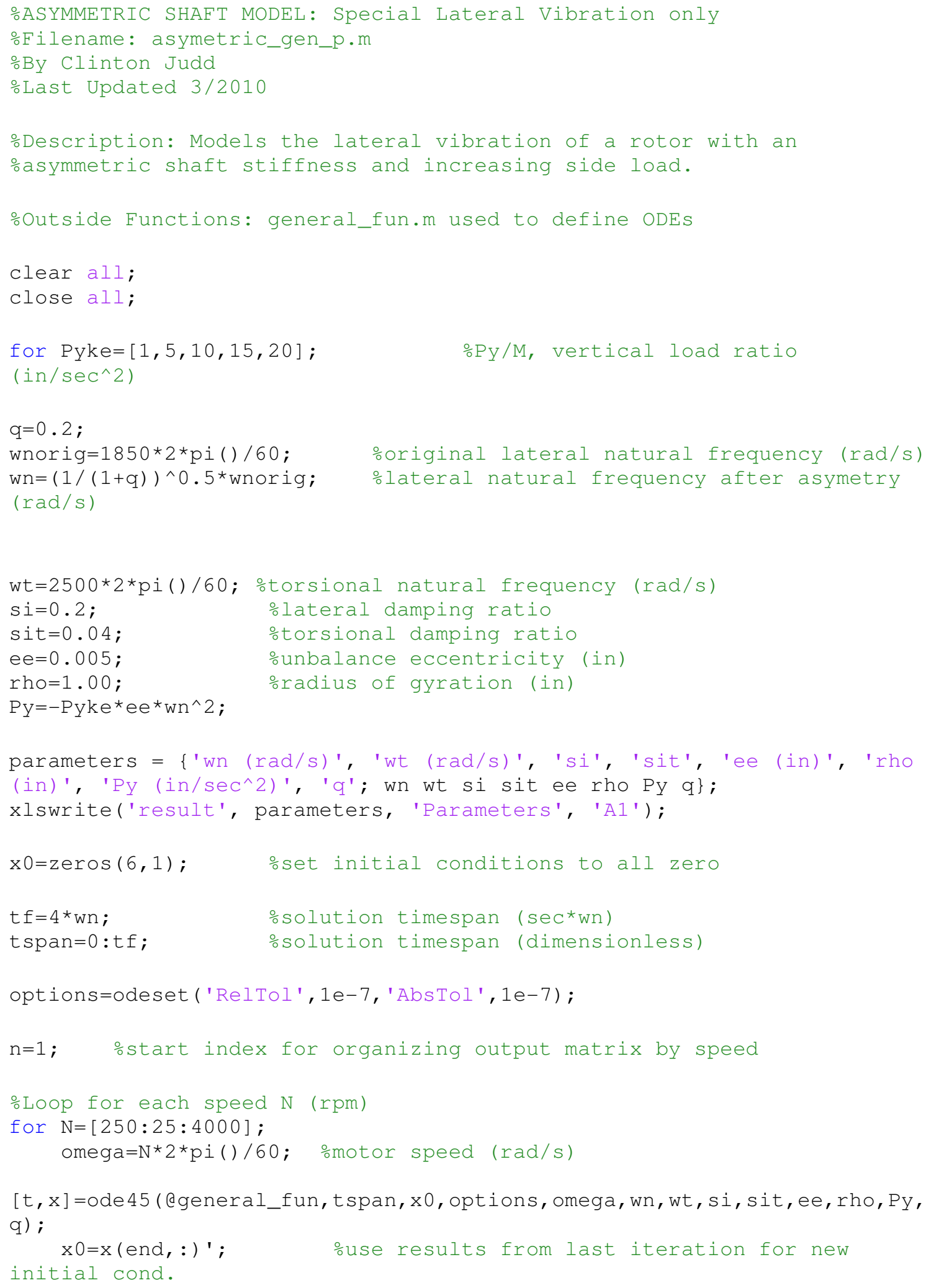




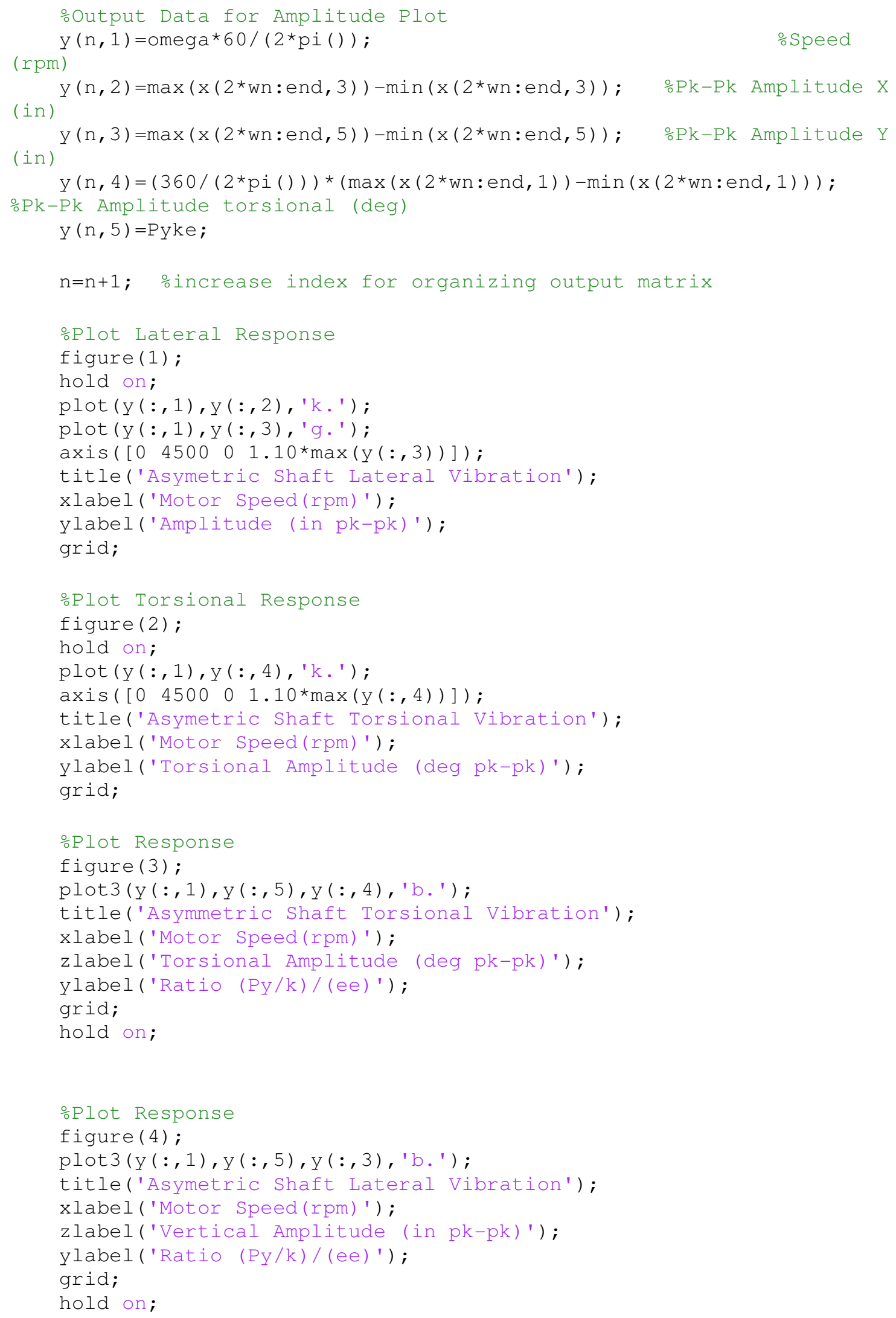


if omega $==0.5 * \mathrm{wn}$

xlswrite('result', y(:,:), ['Pyke' int2str(Pyke) ' '

int $2 \operatorname{str}(\mathrm{N})]$, 'A1');

elseif omega==wn

xlswrite('result', y(:,:), ['Pyke' int2str(Pyke) ' '

int $2 \operatorname{str}(\mathrm{N})]$, 'A1');

end

end 


\section{Appendix D Experiment Trial Notes}

\section{D.1 Notes on Experimental Trial Apparatus}

System consists of two 800g disks as an inboard rotor and two 800g disks as an outboard rotor.

Inboard rotor supported closely by bearings.

Outboard rotor has bearing span of 7.75in

Outboard rotor has asymmetry with 1.5 " long flats ground on either side of rotor.

Flats reduce diameter to a 0.230 in thickness.

Shaft length between rotors is 14in.

Outboard rotor has vertical loading system (pulleys) with masses hung to produce vertical side load.

Prox-probes for lateral vibration measurement near bearings (inboard / outboard)

Gear wheels at each end for torsional measurement (TK17 inboard / outboard)

Calculated $\mathrm{wn}=5,800 \mathrm{rpm}$ and $\mathrm{wt}=4,100 \mathrm{rpm} \mathrm{wt} / \mathrm{wn}=0.707$

$20 \mathrm{lb}$ hung for side loading $\left(\mathrm{Py} / \mathrm{M}=6.7 \mathrm{~g}\right.$ 's $\left.=2600 \mathrm{in} / \mathrm{sec}^{\wedge} 2\right)$ 